\title{
Linearizaçōes Externas e Pontos Interiores em Programação Convexa
}

\author{
Lucia Rosana Moura
}

DISSERTAÇÃO APRESENTADA

$\mathrm{AO}$

INSTITUTO DE MATEMÁTICA E ESTATÍSTICA

DA

UNIVERSIDADE DE SÃO PAULO

PARA OBTENCAAO DO GRAU DE

MESTRE EM

MATEMÁTICA APLICADA

Área de Concentração: Ciência da Computação Orientador: Prof. Dr. Carlos Humes Jr.

\author{
Durante a elaboração deste trabalho. \\ - autor recebe apoio financeiro da CAPES
}

-São Paulo. Agosto de 1992- 
Às páginas em branco, nas quais idéias podem se materializar 


\section{Resumo}

Neste trabalho estudamos um novo algoritmo desenvolvido por Goffin e Vial, para resolver o problema de programação convexa sujeito a restrições lineares. Este algoritmo é um método de planos de corte, onde os cortes são obtidos por linearizações da função objetivo. Ao invés de obter a solução de cada sub-problema linearizado, como em métodos clássicos, este método obtém seu centro analítico, que é um ponto mais fácil de computar e cujas propriedades garantem a convergência do método.

Como todos os métodos de planos de corte, o algoritmo estudado caracteriza-se pelo acréscimo de uma restrição a cada iteração, aumentando muito o tamanho dos sub-problemas. O objetivo deste trabalho foi desenvolver estratégias de eliminação de restrições para o algoritmo de Goffin-Vial. A eliminação das restrições é feita de modo a não perder as propriedades de convergência, e baseia-se em idéias desenvolvidas por Eaves e Zangwill para métodos gerais de planos de corte.

Apresentamos resultados computacionais para funções quadráticas convexas, sujeitas a restrições de box, obtendo bons resultados para as estratégias de eliminação propostas.

\section{Abstract}

In this work we study a new algorithm developed by Goffin and Vial for solving convex programming problems, subject to linear constraints. This algorithm is a cutting plane method, in which cuts are obtained through linearizations of the objective function. Unlike other classical methods, which solve the linearized subproblems each iteration, this one finds an analytic center, an easier point to compute with properties that assure the convergence of the algorithm.

Like other cutting plane methods, the Goffin-Vial algorithm adds a new constraint each iteration, which increases substantially the size of the subproblems. The aim of this work is to develop constraint dropping strategies for this algorithm, maintaining its convergence properties. These strategies are based on ideas introduced by Eaves and Zangwill for general cutting plane methods.

We also present computational results for quadratic convex functions, subject to box constraints. Our numerical tests show advantages in using the proposed constraint dropping strategies. 


\section{Agradecimentos}

Bem, pessoal, tentarei gastar só uma página!

Ao Carlos Humes Jr. por muito mais que uma orientação competente, por gostar de discutir em cima de desenhos malucos que nenhuma pessoa sã entenderia, pela confiança depositada no final do trabalho, por sua sensibilidade para com as pessoas e pela amizade que sobrou desses anos todos de trabalho.

Ao Clóvis Gonzaga, pela inspiração transmitida por sua clareza didática.

Bom, Daniel, por onde começar? Pela força toda fundamental na realização desse trabalho, seu pique, entusiasmo, carinho e companheirismo.

Ao Natan, pelo que ele é, pela energia que me deu desde o seu surgimento neste planeta e por suportar bem a ausência materna das últimas semanas e me receber sempre sorrindo.

À Wandinha e ao Álvaro, meus pais, por tudo e também pelo carinho e cuidado com Natan nesses últimos tempos.

Ao Tito, que ao invés de cuidar da tese dele, nos acompanhou nessas varadas de noite malucas, revendo o texto, digitando, fazendo sugestões e que agora está lá montando o apêndice.

Aos professores Ana Friedlander e José Mário Martinez, por sua atenção e também por cederem o software que permitiu a geração da massa de testes aqui utilizada.

Ao Ricardo Duarte, que cedeu o software de geração de funções lineares num clima bastante simpático via e-mail.

Ao Saulo, pelas consultorias numéricas na arte de domar os erros e pelas 
consultorias fortrânicas na arte de sujar as mãos.

Ao Leojb, pela confeç̧ão de $90 \%$ dos desenhos desse texto, feitos com aquele sorriso nos lábios.

À WanWan, pelas quebradas de galho na sala das ET's, pelo programa de emissão de relatórios e sua preocupação com os outros.

Ao Rinaldo do MAE, por perder duas tardes instalando e ensinando a usar aquela carroça chamada StatGraph, utilizada para confeccionar os gráficos.

Ao Roberto Duó, pela ajuda na confeç̧ão nas tabelas.

Ao Arnaldo, pelo cuidado com os "algogos", muitas vezes fazendo malabarismo para nâo precisar rebootar as máquinas e outras vezes vindo no fim de semana para religar a rede.

Ao Steve, pela revisão do abstract.

À CriCri pela companhia em tantos eventos compartilhados, inclusive um fim de tese.

$\grave{A}$ Dilma pelo entusiasmo que ela consegue transmitir mesmo a milhas de distância.

Ao Kunio e à Nami pela prestatividade.

Ao Paulo Cordaro pela condescendência.

Aos meus irmãos, Rejane, Pedro e Márcia, por poder contar com eles.

Finalmente, a todos os amigos que me acompanharam durante o desenvolvimento do trabalho, e não foram citados porque...já estourei a segunda página!!! 


\section{Conteúdo}

$\begin{array}{ll}\text { Introdução } & 1\end{array}$

1 Pontos Interiores Em Programação Linear 3

1.1 Introdução . . . . . . . . . . . . . 3

1.2 Barreiras, Pontos Centrais e Trajetória Central . . . . . . . 5

1.3 Determinação de um ponto próximo ao centro analítico . . . . 11

2 Algoritmo de Goffin-Vial para Programação Convexa 18

2.1 Propriedades de funções convexas . . . . . . . . . . . 21

2.2 Algoritmos de Planos de Corte . . . . . . . . . . . . . . 31

2.3 Descrição do algoritmo de Goffin-Vial . . . . . . . . . . 35

2.3.1 Algoritmo para o caso linear . . . . . . . . . . . 35

2.3.2 Algoritmo para o caso convexo. . . . . . . . . . 38

2.3.3 Convergência . . . . . . . . . . . . 43

2.3.4 Definição de um critério de parada prático . . . . . . 50

3 Eliminação de restrições $\quad \mathbf{5 3}$

3.1 Garantia de convergência . . . . . . . . . . . . . 54

3.2 Controle de eliminação de restrições . . . . . . . . . 59

4 Resultados Computacionais $\quad 62$

4.1 Aspectos de implementação . . . . . . . . . . . . 6 63

4.1.1 Inicialização da rotina de cálculo do centro . . . . . . 63

4.1.2 Cálculo do centro analítico . . . . . . . . . . . . 64

4.1.3 Critério de parada do algoritmo de Goffin-Vial . . . . 65

4.2 Problemas Teste. . . . . . . . . . . . . . 65 65

4.2 .1 Geração dos dados . . . . . . . . . . . . 65 
4.2 .2 Variações utilizadas nos testes . . . . . . . . . 68

4.3 Algoritmo sem eliminações: efeito dos parâmetros que definem as quadráticas . . . . . . . . . . . . . . 69

4.4 Efeito de eliminação de restrições sobre o algoritmo . . . . . 82 4.4.1 Estratégia de eliminação $1 \ldots \ldots$. . . . . . . 84

4.4 .2 Estratégia de eliminação $2 \ldots \ldots \ldots$. . . . . . . 90

4.4.3 Estratégia de eliminação $3 \ldots \ldots$. . . . . . . . 95

4.4.4 Estratégia de eliminaçâo $4 \ldots \ldots$. . . . . . . 100

4.4.5 Conclusões. . . . . . . . . . . . . . . . . 105

A Gráficos sobre os parâmetros das quadráticas 106

B Gráficos para eliminações 1 e $2 \quad 121$

$\begin{array}{ll}\text { Bibliografia } & 134\end{array}$ 


\section{Introdução}

Neste trabalho estudamos um novo algoritmo desenvolvido por Goffin e Vial [6], para resolver o problema de programação convexa sob restrições lineares definido por:

$$
(P C) \quad\left\{\begin{array}{l}
\min \varphi(x) \\
x \in S=\left\{x \in \mathbb{R}^{n}: A x \leq b\right\}
\end{array}\right.
$$

onde $A \in \mathbb{R}^{m \times n}, b \in \mathbb{R}^{m}, S$ é limitado com interior não vazio e $\varphi(\cdot)$ é convexa em $\mathbb{R}^{n}$.

Este algoritmo é um método de planos de corte, onde os cortes são obtidos por linearizações da função objetivo. Cada sub-problema considera o conjunto obtido pelos cortes, e determina um ponto interior com propriedades interessantes chamado centro analítico. Centros analíticos têm sido utilizados em algoritmos de trajetória central para programação linear, que são uma classe de métodos pertencente aos métodos de pontos interiores. A demonstração da convergência do método foi primeiro apresentada por Gonzaga [7].

Como todos os métodos de planos de corte, o algoritmo estudado caracterizase pelo acréscimo de uma restrição a cada iteração, aumentando muito o tamanho dos sub-problemas. O objetivo deste trabalho foi desenvolver estratégias de eliminação de restriçôes para o algoritmo de Goffin-Vial. Estas estratégias se baseiam em idéias desenvolvidas por Eaves e Zangwill [3] para métodos gerais de planos de corte, adaptando-as ao algoritmo estudado. 
As eliminações de restrições são feitas quando certas condições em um passo do algoritmo são satisfeitas. Como não há garantia de quando isto ocorre para o problema geral $(P C)$, não podemos provar teoricamente qual o efeito das estratégias de eliminação sobre o método.

Para termos uma idéia desse efeito, implementamos o algoritmo original com e sem os métodos de eliminação, para um certo tipo de problemas, obtendo bons resultados.

O trabalho está organizado como descrevemos a seguir:

No capítulo 1 descreveremos, em linhas gerais, algoritmos de trajetória central para programação linear, dando um enfoque especial à determinação de centros analíticos e suas propriedades, que serão úteis na definição do algoritmo de Goffin-Vial.

No capítulo 2 descreveremos o algoritmo de Goffin-Vial, demonstrando sua convergência, com argumentos baseados na demonstraçâo de Gonzaga [7]. Para tanto, apresentaremos inicialmente alguns resultados clássicos de funçôes convexas e um "approach" geral para métodos de planos de corte.

No capítulo 3 trataremos da descrição das estratégias de eliminação propostas, demonstrando que a convergência do algoritmo é mantida.

No capítulo 4 analisaremos os resultados computacionais obtidos com a introdução das eliminaçôes de restrições para exemplos de funções quadráticas convexas sujeitas a restrições de "box" geradas aleatoriamente. 


\section{Capítulo 1}

\section{Pontos Interiores Em Programação Linear}

\subsection{Introdução}

Consideremos o problema de programação linear:

$$
(P L) \quad\left\{\begin{array}{l}
\min c^{T} x \\
x \in S=\left\{x \in \mathbb{R}^{n}: A x \leq b\right\}
\end{array}\right.
$$

onde $A \in \mathbb{R}^{m \times n}, b \in \mathbb{R}^{m}, S$ é limitado com interior não vazio e $c \in \mathbb{R}^{n}$.

Este problema por muito tempo foi resolvido pelo método Simplex de Dantzig [1] que percorre vértices do poliedro $S$ até encontrar um vértice ótimo. Este método mostrou-se bastante eficiente na prática, apesar de ter do ponto de vista teórico uma característica indesejável: no pior caso o método percorre todos os vértices do poliedro, tendo complexidade exponencial.

Esta questão teórica só foi resolvida em 1978 com o algoritmo dos Elipsóides de Khachiyan [12] que provou que o $(P L)$ podia ser resolvido com complexidade polinomial no "tamanho do problema" $(L=n b+m+1$, onde $n b$ é o número de bits necessários para representar todas as entradas do problema e $m$ é a dimensão do espaço). Este método utilizava uma idéia bastante 
diferente do método Simplex: percorria pontos no interior do poliedro. Apesar de, em termos de complexidade de pior caso o método dos elipsóides ser melhor que o simplex, este mostrou-se bem mais eficiente para muitos problemas práticos.

Apenas em 1984 surgiu um método com complexidade polinomial que mostrouse eficiente na prática: o algoritmo de Karmarkar [11]. O aparecimento deste método gerou muita agitação na área, fazendo com que muitos pesquisadores se dedicassem a compreender as idéias que haviam por trás do método, já que sua formulação original era bastante difícil e obscura. O método tinha complexidade $\mathcal{O}((m+n) L)$ iterações, $\mathcal{O}\left((m+n)^{3.5} L\right)$ operações aritméticas e era um método de pontos interiores que se baseava em uma transformação não linear chamada "transformação projetiva" e numa função auxiliar chamada "função potencial".

O método de Karmarkar resolvia o problema no formato padrão (problema dual do $(P L)$ escrito no início desta seção), e além disso, inicialmente, haviam uma série de hipóteses restritivas. Estas hipóteses foram posteriormente aliviadas e uma série de variações foram feitas por vários pesquisadores.

O primeiro algoritmo de trajetória central com complexidade de iterações em número inferior ao algoritmo de Karmarkar foi desenvolvido por Renegar [16]. O método utilizava $\mathcal{O}(\sqrt{m+n} L)$ iterações e mesma complexidade que Karmarkar em número de operações aritméticas. Apesar de não melhorar a complexidade de Karmarkar, o método tinha uma importante característica: era conceitualmente bem mais simples e tinha um forte apelo geométrico.

Outros métodos de trajetória central foram desenvolvidos posteriormente, e um interessante "survey" destes métodos pode ser visto em Gonzaga [9]. Esta trajetória é uma curva suave formada por pontos que são "centrais" num certo sentido. As idéias utilizadas neste tipo de algoritmo bem como as definições necessárias serão vistas na próxima seção. 


\subsection{Barreiras, Pontos Centrais e Trajetória Central}

Os métodos de pontos interiores combinam duas idéias principais a cada iteração : diminuição da função objetivo e garantia de permanecer no interior do conjunto. Métodos que se aproximam demais da fronteira do conjunto, como o afim-escala [8], apesar de eficientes na prática, não permitiram até o momento uma garantia de complexidade polinomial. O afim-escala procura uma "vantagem imediata" em termos de redução da função objetivo sem preocupar-se com futuras iterações.

Todos os métodos de pontos interiores combinam direções que garantem "centralidade" com a direção de minimização da função objetivo. Um papel fundamental na garantia de "centralidade" tem a função barreira, descrita a seguir.

A função barreira foi primeiramente usada por Frisch [5], num método de programação não linear chamado método de barreiras. A função barreira $p: \rightarrow \mathbb{R}$ é definida por:

$$
p(x)=-\sum_{i=1}^{n} \ln \left(b_{i}-a_{i} x\right)
$$

onde $a_{i}$ é a linha $i$ da matriz $A$, para o problema no formato $(P L)$. Note que se escrevemos $(P L)$ da seguinte forma:

$$
(P L)=\left\{\begin{array}{l}
\min c^{T} x \\
A x+z=b \\
z \geq 0
\end{array}\right.
$$

a função barreira pode ser escrita como: $p(z)=-\sum_{i=1}^{n} \ln \left(z_{i}\right)$

Esta variável $z$ é chamada de "variável de folga" do $(P L)$ já que ela está relacionada à distância $d_{i}$ de um ponto $x$ ao hiperplano $a_{i} x=b_{i}\left(z_{i}=d_{i}\left\|a_{i}\right\|\right)$. As curvas de nível da função barreira para um problema no formato $(P L)$ podem ser ilustradas como na figura abaixo. 


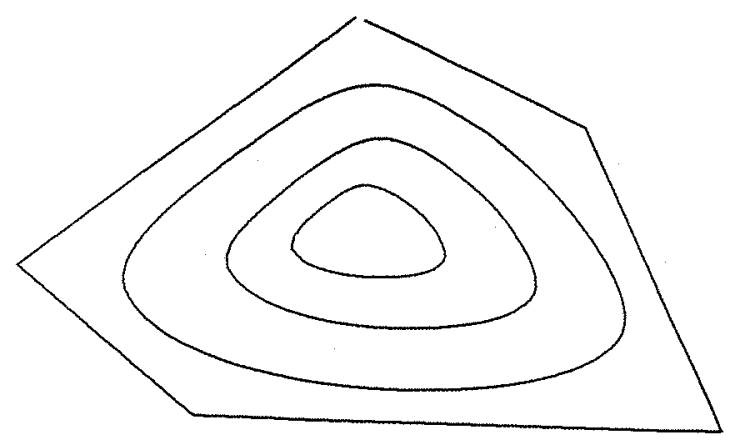

Figura 1.1: Curvas de nivel da função barreira

Observe que a função barreira penaliza pontos que se aproximam demais da fronteira do conjunto, já que quanto menor é a folga, menor será o seu logaritmo e imediatamente maior será o valor da função barreira.

O centro analítico de $S$ é o único ponto que minimiza a função barreira:

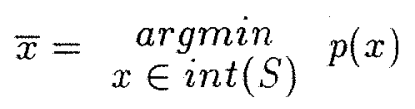

É fácil ver que $\bar{x}$ é único já que $p$ é estritamente convexa em $\operatorname{int}(S)$.

Este ponto foi definido por Sonnevend [18]. Centros analíticos diferem essencialmente de centros geométricos pois dependem da descrição analítica da região. Um mesmo poliedro se for descrito de uma outra forma, por exemplo repetindo-se uma restrição várias vezes, terá um centro analítico diferente. A restrição que foi repetida na descrição analítica do poliedro aparecerá várias vezes na função barreira causando uma "repulsão" no centro analítico que tenderá a ficar deslocado com relação a essa restrição. Esta dependência da descrição analítica de $S$ para determinação do centro pode ser vista na figura abaixo: 

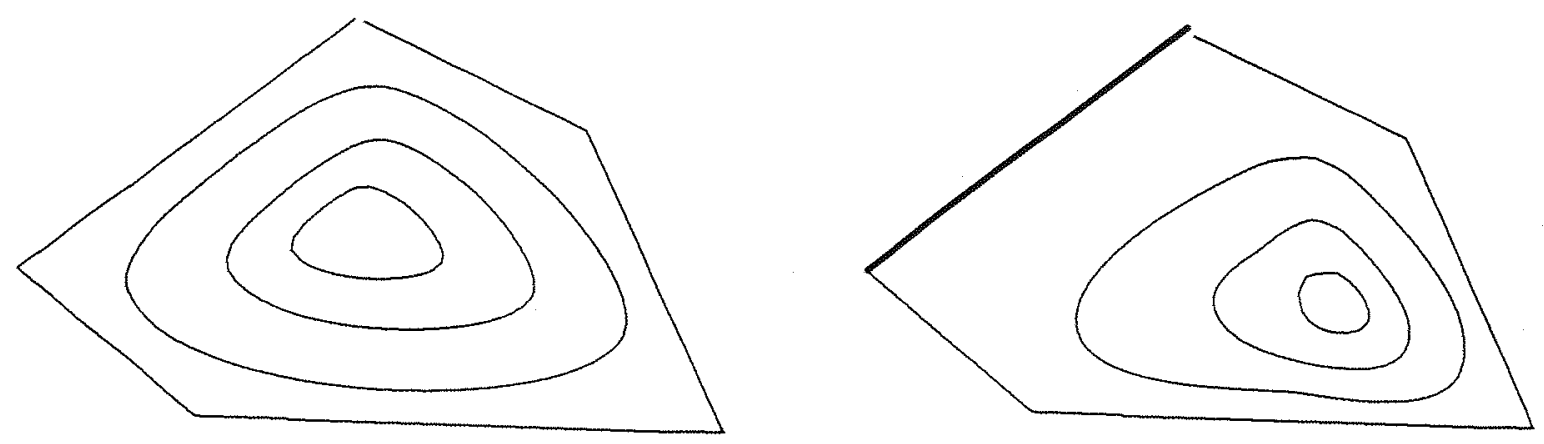

Figura 1.2: Variaçào do centro analítico por duplicaçâo de restriçâo

Centro de gravidade e centro do maior elipsóide inscrito no poliedro sâo exemplos de centros geométricos. O problema em utilizar estes centros é que eles são muito difíceis de computar.

$\mathrm{O}$ centro analítico nào pode ser calculado exatamente mas podemos obter um ponto próximo do centro analítico em tempo polinomial.

Agora desejamos combinar estas idéias de centralidade com a redução da funçào objetivo. Queremos reduzir o custo, mas ao mesmo tempo nos manter no "centro" do poliedro, num certo sentido.

Considere os conjuntos:

$S_{u}=\left\{x \in S: c^{T} x \leq u\right\}$ que tem interior não vazio e as funçòes centro: $f_{u}: \operatorname{int}\left(S_{u}\right) \rightarrow \mathbb{R}$ definidas por:

$$
f_{u}(x)=-q \ln \left(u-c^{T} x\right)-\sum_{i=1}^{m} \ln \left(b_{i}-a_{i} x\right)
$$

Observe que $f_{u}$ corresponde à função barreira de $S_{u} \operatorname{com}$ a restriçãc $\varepsilon^{T} x \leq u$ repetida $q$ vezes, e o centro analítico desta região é dado por:

$$
\bar{x}(u)=\underset{x \in \operatorname{argmin}}{\arg \left(S_{u}\right)} f_{u}(x)
$$


Estes pontos são chamados pontos centrais e a curva formada por estes é chamada trajetória central.

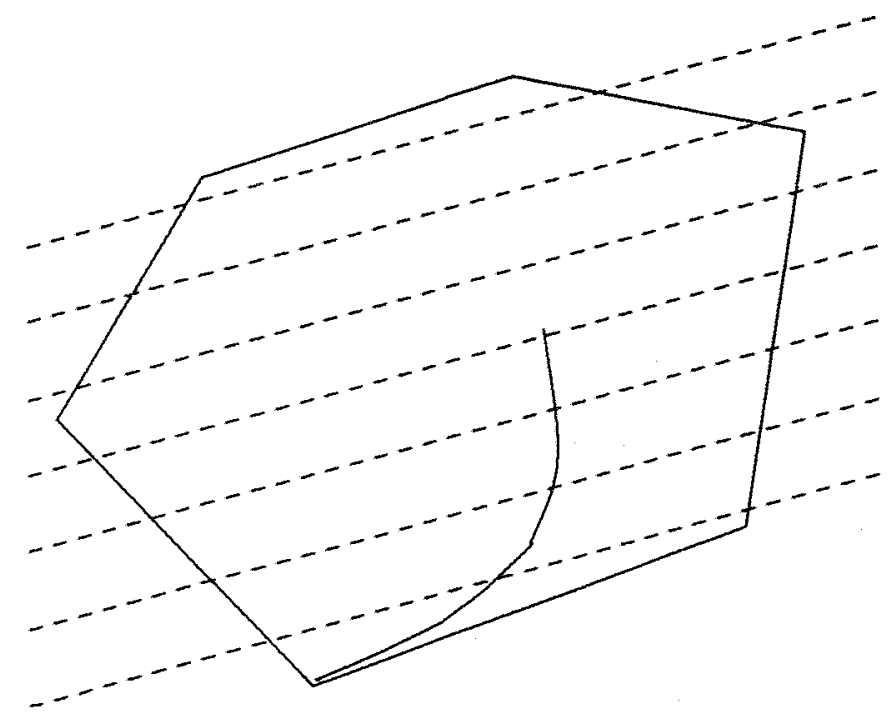

Figura 1.3: Trajetória central e curvas de nível da função linear

Dado $u$, o teorema abaixo mostra uma interessante relação entre o valor da função objetivo no centro analítico de $S_{u}$ e o valor ótimo do problema de programação linear.

Teorema 1.1 Se $\bar{x}=\bar{x}(u)$ é centro analítico de $S_{u}$ e $x^{*}$ é solução ótima de:

$$
(P L) \quad\left\{\begin{array}{l}
\min c^{T} x \\
x \in S
\end{array}\right.
$$

Então

$$
c^{T} \bar{x}-c^{T} x^{*} \leq \frac{m\left(u-c^{T} \bar{x}\right)}{q}
$$




\section{Demonstração}

Pelas condições de gradiente nulo de Karush-Kuhn-Tucker, como $\bar{x}$ minimiza $f_{u}$, temos que:

$$
\nabla f_{u}(\bar{x})=\frac{q}{u-c^{T} \bar{x}} c+\sum_{i=1}^{m} \frac{1}{b_{i}-a_{i} \bar{x}} a_{i}^{T}=0
$$

Multiplicando a expressão por $\frac{u-c^{T} \bar{x}}{q}$, temos:

$$
c+\sum_{i=1}^{m} \frac{u-c^{T} \bar{x}}{q\left(b_{i}-a_{i} \bar{x}\right)} a_{i}^{T}=0
$$

Escrevendo o dual de Wolfe de $(P L)$ :

$$
(P D) \quad\left\{\begin{array}{l}
\max c^{T} x-\sum y_{i}\left(b_{i}-a_{i} x\right) \\
A x \leq b \quad y \geqq 0 \\
A^{T} y+c=0
\end{array}\right.
$$

Chamando $\quad \bar{y}_{i}=\frac{u-c^{T} \bar{x}}{q\left(b_{i}-a_{i} \bar{x}\right)}$

Por $(I)$ temos que $c+A^{T} \bar{y}=0$. Portanto $(\bar{x}, \bar{y})$ é viável no dual de Wolfe, e pelo teorema fraco de dualidade:

Valor Objetivo do Dual em $(\bar{x}, \bar{y}) \leq$ Valor Ótimo do Primal

$$
\begin{aligned}
& c^{T} \bar{x}-\sum_{i=1}^{m} \bar{y}_{i}\left(b_{i}-a_{i} \bar{x}\right) \leq c^{T} x^{*} \\
& c^{T} \bar{x}-\sum_{i=1}^{m} \frac{u-c^{T} \bar{x}}{q\left(b_{i}-a_{i} \bar{x}\right.}\left(b_{i}-a_{i} \bar{x}\right) \leq c^{T} x^{*} \\
& c^{T} \bar{x}-\frac{u-c^{T} \bar{x}}{q} \sum_{i=1}^{m} \frac{b_{i}-a_{i} \bar{x}}{b_{i}-a_{i} \bar{x}} \leq c^{T} x^{*} \\
& c^{T} \bar{x}-\frac{u-c^{T} \bar{x}}{q} m \leq c^{T} x^{*} \\
& c^{T} \bar{x}-c^{T} x^{*} \leq \frac{m\left(u-c^{T} \bar{x}\right)}{q}
\end{aligned}
$$

O corolário abaixo mostra que com uma escolha conveniente de $q$, podemos garantir um decréscimo relativo a $u$ no valor objetivo de $(P L)$ em $\bar{x}$. 
Corolário 1.1 Sejam $p>1, q \geq m(p-1), \bar{x}$ centro analítico de $S_{u}$ e $x^{*}$ valor ótimo do $(P L)$. Então

$$
c^{T} \bar{x} \leq c^{T} x^{*}+\frac{u-c^{T} x^{*}}{p}
$$

Demonstração Pelo teorema e como $q \geq m(p-1)$

$$
c^{T} \bar{x}-c^{T} x^{*} \leq \frac{m\left(u-c^{T} \bar{x}\right)}{q} \leq \frac{m\left(u-c^{T} \bar{x}\right)}{m(p-1)}=\frac{u-c^{T} \bar{x}}{p-1}
$$

Portanto

$$
\begin{aligned}
& c^{T} \bar{x}-c^{T} x^{*} \leq \frac{u-c^{T} \bar{x}}{p-1} \\
& c^{T} \bar{x}+\frac{c^{T} \bar{x}}{p-1} \leq c^{T} x^{*}+\frac{u}{p-1} \\
& p c^{T} \bar{x} \leq(p-1) c^{T} x^{*}+u \\
& c^{T} \bar{x} \leq c^{T} x^{*}+\frac{u-c^{T} x^{*}}{p}
\end{aligned}
$$

O corolário acima mostra que a diferença entre o valor da função objetivo em $\bar{x}$ e o valor ótimo está limitada por uma fração da diferença entre o limitante superior $u$ e o valor ótimo. A figura abaixo mostra esta relação para $p=2$ :

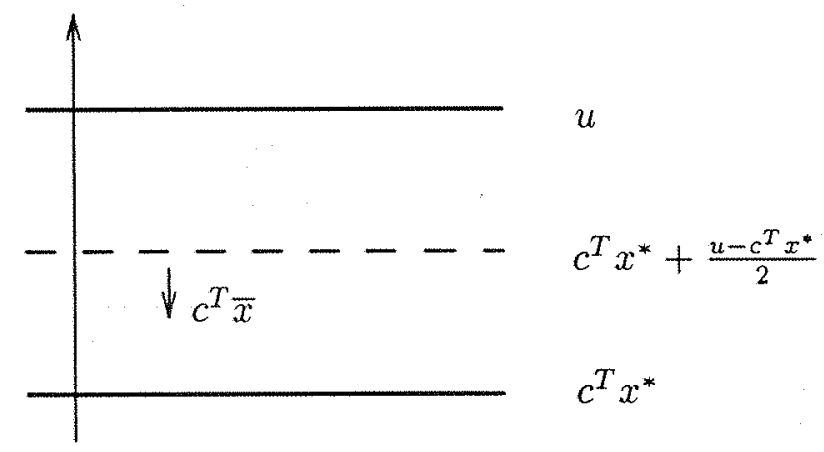


Algoritmos de trajetória central geram pontos "próximos" à trajetória central, já que não é possível encontrar exatamente um ponto central em tempo finito. Um critério preciso de "proximidade" à trajetória central deve ser definido, para podermos garantir um decréscimo relativo a $u$ para um ponto próximo de $\bar{x}$.

Um método de trajetória central genérico pode ser descrito como abaixo:

\section{Algoritmo de Trajetória Central}

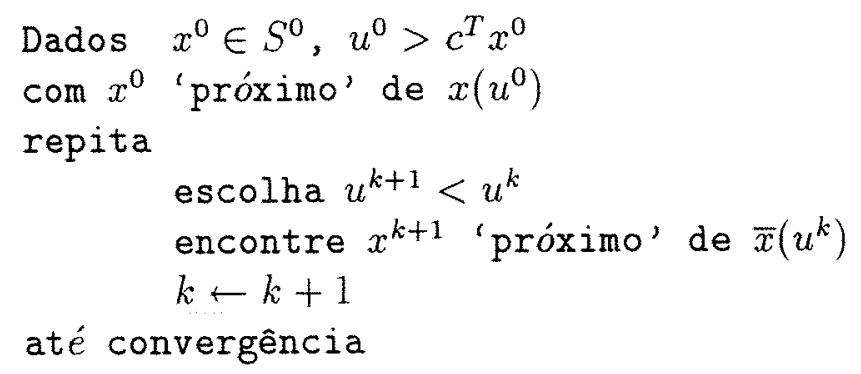

Os algoritmos vão depender da escolha de $q$ na definição da função centro, da inicialização $\left(u^{0}, x^{0}\right)$, e principalmente da atualização de $u^{k}$.

A definição de um critério prático de proximidade ao centro analítico será vista na próxima seção.

\subsection{Determinação de um ponto próximo ao centro analítico}


Para determinar um ponto próximo a $\bar{x}(u)$, devemos resolver aproximadamente:

$$
\left(P_{u}\right) \quad\left\{\begin{array}{l}
\min f_{u}(x)=-q \ln \left(u-c^{T} x\right)-\sum_{i=1}^{m} \ln \left(b_{i}-a_{i} x\right) \\
x \in \operatorname{int}\left(S_{u}\right)
\end{array}\right.
$$

onde $S_{u}=\left\{x \in \mathbb{R}^{n}: A x \leq b, c^{T} x \leq u\right\}$.

Chamando:

$$
\bar{A}=\left[\begin{array}{l}
A \\
c^{T}
\end{array}\right] \quad \bar{b}=\left[\begin{array}{l}
b \\
u
\end{array}\right]
$$

temos que $S_{u}$ é dado por $S_{u}=\left\{x \in \mathbb{R}^{n}: \bar{A} x \leq \bar{b}\right\}$.

Consideremos a aproximação quadrática de $f_{u}(\cdot)$ em torno do ponto $x^{\prime}$ :

$$
Q(x)=f_{u}\left(x^{\prime}\right)+\nabla f_{u}\left(x^{\prime}\right)\left(x-x^{\prime}\right)+\frac{1}{2}\left(x-x^{\prime}\right)^{T} \nabla^{2} f_{u}\left(x^{\prime}\right)\left(x-x^{\prime}\right)
$$

onde $\nabla f_{u}\left(x^{\prime}\right)$ é o gradiente de $f_{u}(\cdot)$ em $x^{\prime}$ e $\nabla^{2} f_{u}\left(x^{\prime}\right)$ é a matriz hessiana de $f_{u}(\cdot)$ em $x^{\prime}$.

Seja $x^{*}$ o ponto que minimiza a aproximação quadrática de $f_{u}(\cdot)$. Como $f_{u}(\cdot)$ é convexa então $\nabla^{2} f_{u}(\cdot)$ é definida positiva, portanto $x^{*}$ minimiza $Q(x) \Longleftrightarrow \nabla Q\left(x^{*}\right)=0$. Mas $\nabla Q(x)=\nabla f_{u}\left(x^{\prime}\right)+\nabla^{2} f_{u}\left(x^{\prime}\right)\left(x-x^{\prime}\right)$, portanto

$$
\nabla Q\left(x^{*}\right)=\nabla f_{u}\left(x^{\prime}\right)+\nabla^{2} f_{u}\left(x^{\prime}\right)\left(x^{*}-x^{\prime}\right)=0
$$

Logo a direção de minimização da aproximação quadrática a partir de $x^{\prime}$ é dada por:

$$
h=x^{*}-x^{\prime}=-\left(\nabla^{2} f_{u}\left(x^{\prime}\right)\right)^{-1} \nabla f_{u}\left(x^{\prime}\right)
$$

Utilizaremos o método de Newton-Raphson que faz sucessivas minimizações unidirecionais nas direções de minimização da aproximação quadrática, como descrito a seguir. 


\section{Algoritmo de Newton-Raphson}

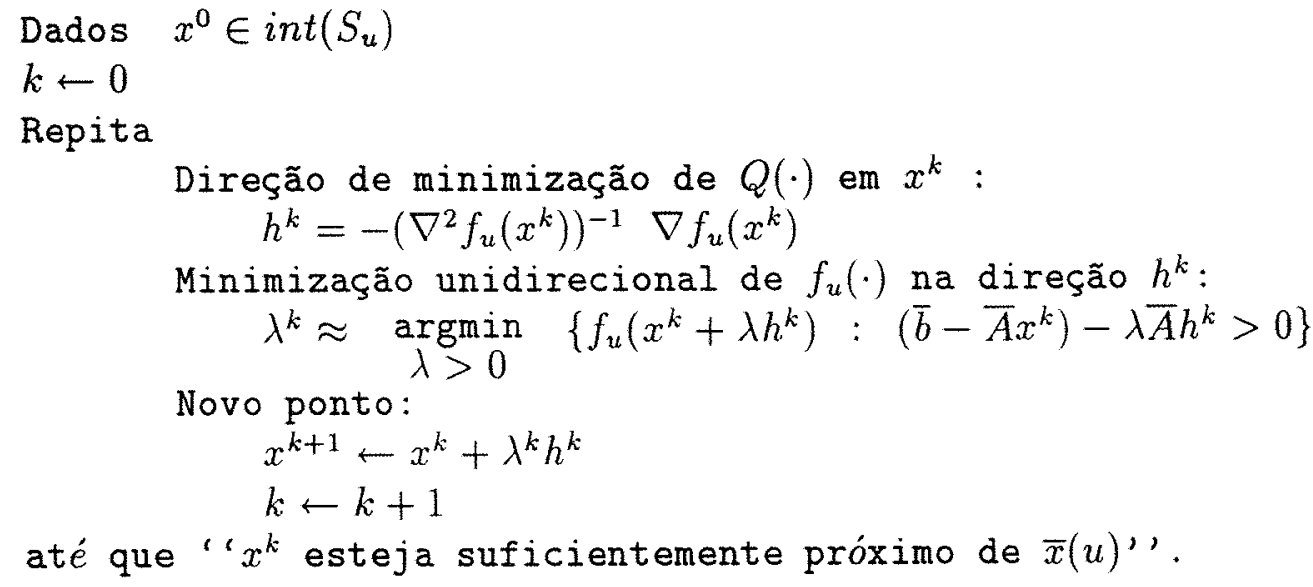

O critério de parada do algoritmo precisa ser melhor definido, já que não conhecemos o centro analítico $\bar{x}(u)$. A seguir será definida uma medida de proximidade útil na prática, pois utiliza apenas a direção $h^{k}$ e a hessiana $\nabla^{2} f_{u}\left(x^{k}\right)$

\section{Medida de Proximidade}

A seguir faremos alguns cálculos que serão úteis posteriormente:

$$
\begin{gathered}
g=g(x, u)=\nabla f_{u}(x)=\frac{q}{u-c^{T} x} c+\sum_{i=1}^{m} \frac{1}{b_{i}-a_{i} x} a_{i}^{T} \\
H=H(x, u)=\nabla^{2} f_{u}(x)=\frac{q}{\left(u-c^{T} x\right)^{2}} c c^{T}+\sum_{i=1}^{m} \frac{1}{\left(b_{i}-a_{i} x\right)^{2}} a_{i}^{T} a_{i}
\end{gathered}
$$

Definição 1.1 Dado $x \in \mathbb{R}^{n}$ e $H$ matriz $n \times n$ definida positiva, a norma de $x$ relativa a $H$ é dada por:

$$
\|x\|_{H}=\sqrt{x^{T} H x}
$$


Usaremos como medida de proximidade de um ponto $x$ ao centro analítico $\bar{x}(u)$, a seguinte norma:

$$
\delta(x, u)=\|h\|_{H}
$$

onde

$$
h=-\left(\nabla^{2} f_{u}(x)\right)^{-1} \nabla f_{u}(x)
$$

é a direção de minimização da aproximação quadrática de $f_{u}(\cdot)$ em $x$ e

$$
H=\nabla^{2} f_{u}(x)
$$

é a matriz hessiana de $f_{u}(\cdot)$ em $x$.

As proposições que vêm a seguir, mostrarão que $\delta(x, u)=\|h\|_{H}$ é uma boa medida de proximidade ao centro analítico.

Proposição 1.1 Sejam $x^{\prime} \in S_{u}, x^{*}$ o mínimo da aproximação quadrática de $f_{u}(\cdot)$ em $x^{\prime}$ e $\varepsilon>0$. Chamando $h=h\left(x^{\prime}, u\right)=x^{*}-x^{\prime}, H=H\left(x^{\prime}, u\right), e$ $\bar{x}(u)$ é o centro analítico de $S_{u}$ temos que

se $\delta\left(x^{\prime}, u\right)=\|h\|_{H}<\bar{\delta}<0.5 \quad$ e $\varepsilon=\frac{\bar{\delta}(1-\bar{\delta})(1+2 \bar{\delta})}{(1+\bar{\delta})(1-2 \bar{\delta})}$ então

$$
\left\|x^{\prime}-\bar{x}(u)\right\|_{H} \leq \varepsilon
$$

Demonstração

Pode ser visto em Delgado [2] e Gonzaga [9].

Proposição 1.2 Sejam $\bar{x}(u)$ centro analítico de $S_{u}, x \in S_{u}, H=H(x, u)$ e $\varepsilon>0$. Se $\|x-\bar{x}(u)\|_{H} \leq \varepsilon$ então

$$
\left|c^{T} x-c^{T} \bar{x}(u)\right| \leq \varepsilon \frac{u-c^{T} x}{\sqrt{q}}
$$




\section{Demonstração}

Denotemos $\bar{x}=\bar{x}(u)$.

Pela hipótese $\|x-\bar{x}\|_{H} \leq \varepsilon$, temos que $\|x-\bar{x}\|_{H}^{2} \leq \varepsilon^{2}$.

$$
\begin{aligned}
\varepsilon^{2} & \geq\|x-\bar{x}\|_{H}^{2}=(x-\bar{x})^{T} H(x-\bar{x})=\text { pela definição de } \mathrm{H} \\
& =(x-\bar{x})^{T}\left(\frac{q}{\left(u-c^{T} x\right)^{2}} c c^{T}+\sum_{i=1}^{m} \frac{1}{b_{i}-a_{i} x} a_{i}^{T} a_{i}\right)(x-\bar{x})= \\
& =\frac{q\left(c^{T}(x-\bar{x})\right)^{2}}{\left(u-c^{T} x\right)^{2}}+\sum_{i=1}^{m} \frac{\left(a_{i}(x-\bar{x})\right)^{2}}{\left(b_{i}-a_{i} x\right)^{2}} \geq \frac{q\left(c^{T}(x-\bar{x})\right)^{2}}{\left(u-c^{T} x\right)^{2}}
\end{aligned}
$$

Reescrevendo temos

$$
\frac{q\left(c^{T}(x-\bar{x})\right)^{2}}{\left(u-c^{T} x\right)^{2}} \leq \varepsilon^{2}
$$

Tomando a raiz quadrada,

$$
\sqrt{q}\left|c^{T} x-c^{T} \bar{x}\right| \leq \varepsilon\left(u-c^{T} x\right)
$$

O teorema abaixo mostra um decréscimo no valor objetivo de um ponto $x^{\prime}$ que é uma aproximação do centro analítico do poliedro $S_{u}$ com um peso $q$ conveniente na função centro.

Teorema 1.2 Seja $p>1$ e $x^{*}$ soluçâo ótima do $(P L)$. Então existem $r>1$ e $\varepsilon>0$ tais que

Se $x^{\prime}$ é aproximação do centro analítico $\bar{x}(u)$ de $S_{u}$, com os parâmetros $q \geq$ $(r-1) m$ e $\delta\left(x^{\prime}, u\right) \leq \bar{\delta}<0.5$ então

$$
c^{T} x^{\prime} \leq c^{T} x^{*}+\frac{u-c^{T} x^{*}}{p}
$$




\section{Demonstração}

Tomemos $r>p$ e

$$
\varepsilon \leq \min \left\{\frac{(r-p) \sqrt{(r-1) m}}{r(p-1)}, \frac{\bar{\delta}(1-\bar{\delta})(1+2 \bar{\delta})}{(1+\bar{\delta})(1-2 \bar{\delta})}\right\}
$$

Pela proposição $1.1, \operatorname{como} \delta\left(x^{\prime}, u\right) \leq \bar{\delta} \leq 0.5$, temos $\left\|x^{\prime}-\bar{x}(u)\right\|_{H} \leq \varepsilon$, e pela proposição 1.2 vem que

$$
c^{T} x^{\prime} \leq c^{T} \bar{x}(u)+\Delta\left(u-c^{T} x^{\prime}\right)
$$

onde $\Delta=\frac{\varepsilon}{\sqrt{q}}$, e portanto

$$
c^{T} x^{\prime} \leq \frac{c^{T} \bar{x}(u)+\Delta u}{1+\Delta}
$$

Por outro lado, pelo corolário 1.1 , temos que para $q \geq(r-1) m$

$$
c^{T} \bar{x}(u) \leq c^{T} x^{*}+\frac{u-c^{T} x^{*}}{r}
$$

Substituindo (iii) em (ii), vem que

$$
c^{T} x^{\prime} \leq \frac{c^{T} x^{*}+\frac{u-c^{T} x^{*}}{r}+\Delta u}{1+\Delta}
$$

Reescrevendo temos

$$
c^{T} x^{\prime} \leq \frac{(r-1) c^{T} x^{*}+(1+\Delta r) u}{(1+\Delta) r}
$$

Por outro lado, como $\varepsilon \leq \frac{(r-p) \sqrt{(r-1) m}}{r(p-1)}$ e $q \geq(r-1) m$, temos que

$$
\Delta=\frac{\varepsilon}{\sqrt{q}} \leq \frac{r-p}{r(p-1)}
$$

e consequentemente

$$
(p-1) r \Delta \leq r-p
$$


Multiplicando os dois lados da desigualdade por $\left(u-c^{T} x^{*}\right)$ obtemos

$$
(p-1) r \Delta\left(u-c^{T} x^{*}\right) \leq r\left(u-c^{T} x^{*}\right)-p\left(u-c^{T} x^{*}\right)
$$

Reescrevendo

$$
p u-p c^{T} x^{*}+p \Delta r u \leq((p-1) \Delta r-r) c^{T} x^{*}+(\Delta r+r) u
$$

Somando $p r c^{T} x^{*}$ aos dois lados e reagrupando os termos, vem que

$$
(r-1) p c^{T} x^{*}+p u(1+\Delta r) \leq(p-1)(\Delta r+r) c^{T} x^{*}+r(1+\Delta) u
$$

Dividindo por $(1+\Delta) r p$ temos

$$
\frac{(r-1) c^{T} x^{*}+u(1+\Delta r)}{(1+\Delta) r} \leq \frac{(p-1) c^{T} x^{*}+u}{p}
$$

Por $(I)$, vem que

$$
c^{T} x^{\prime} \leq \frac{(p-1) c^{T} x^{*}+u}{p}=c^{T} x^{*}+\frac{u-c^{T} x^{*}}{p}
$$




\section{Capítulo 2}

\section{Algoritmo de Goffin-Vial para Programação Convexa}

O problema de programação convexa que estamos interessados em resolver é o problema de minimizar uma função convexa sujeita a restrições lineares. Relembrando a definiçâo de função convexa, temos:

Definição 2.1 Uma função $\varphi: \mathbb{R}^{n} \rightarrow \mathbb{R}$ é convexa num ponto $\bar{x}$ se e só se $\forall x \in \mathbb{R}^{n}$ e $\forall \lambda \in[0,1]$ temos que $\varphi(\lambda \bar{x}+(1-\lambda) x) \leq \lambda \varphi(\bar{x})+(1-\lambda) \varphi(x)$ Dizemos simplesmente que $\varphi(\cdot)$ é convexa se ela for convexa em todos os pontos $x \in \mathbb{R}^{n}$.

A figura 2.1 mostra uma função convexa.

O problema de programação convexa é descrito abaixo:

$$
(P C) \quad\left\{\begin{array}{l}
\min \varphi(x) \\
x \in S=\left\{x \in \mathbb{R}^{n}: A x \leq b\right\}
\end{array}\right.
$$

onde $A \in \mathbb{R}^{m \times n}, b \in \mathbb{R}^{m}$ e $\varphi: \mathbb{R}^{n} \rightarrow \mathbb{R}$ convexa.

Supomos que $S$ é limitado com interior não vazio. 


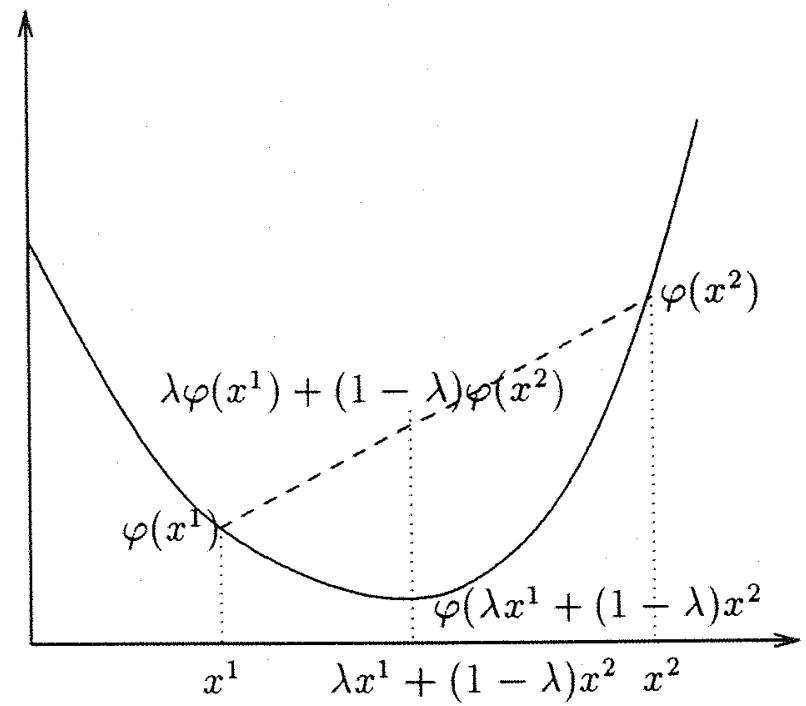

Figura 2.1: Função Convexa

Definição 2.2 Consideremos $\varphi: S \rightarrow \mathbb{R}$. Chamaremos de epígrafe de $\varphi$ ao conjunto:

$$
G_{\varphi}=\{(x, y) \in S \times \mathbb{R}: y \geq \varphi(x)\}
$$

e de gráfico de $\varphi$ ao conjunto:

$$
G_{\varphi}^{=}=\{(x, y) \in S \times \mathbb{R}: y=\varphi(x)\}
$$

A figura abaixo ilustra estes conjuntos: 


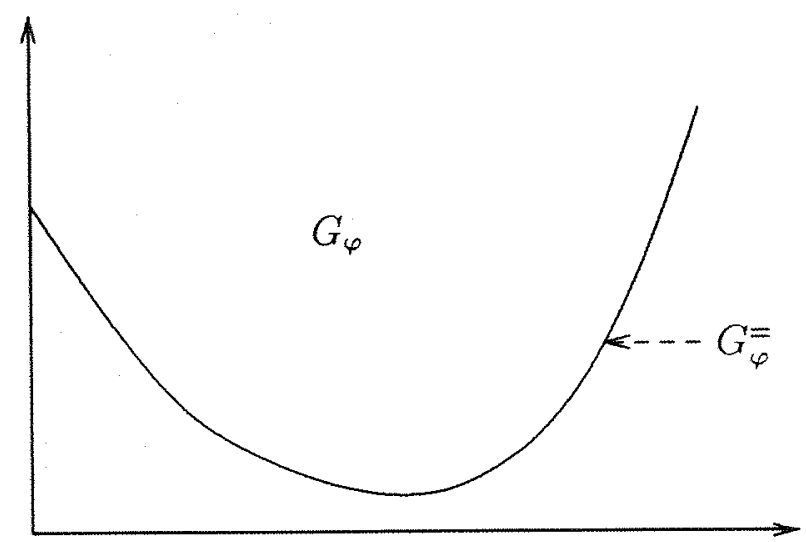

Figura 2.2: Epígrafe e gráfico de $\varphi$

O problema $(P C)$ pode ser reescrito como $\left(P C^{\prime}\right)$

$$
\left(P C^{\prime}\right) \quad\left\{\begin{array}{l}
\min y \\
(x, y) \in G_{\varphi}
\end{array}\right.
$$

Note que $x^{*}$ é solução de $(P C)$ se e somente se $\left(x^{*}, \varphi\left(x^{*}\right)\right)$ é solução de $\left(P C^{\prime}\right)$ pois:

$x^{*}$ é solução de $(P C) \Longleftrightarrow \varphi\left(x^{*}\right) \leq \varphi(x) \forall x \in S \Longleftrightarrow$ $\Longleftrightarrow \varphi\left(x^{*}\right) \leq y \forall y:(x, y) \in G_{\varphi} \Longleftrightarrow\left(x^{*}, \varphi\left(x^{*}\right)\right)$ é solução de $\left(P C^{\prime}\right)$.

Neste capitulo estudaremos o algoritmo de Goffin-Vial para resolver $(P C)$. Ele é um método de planos de corte que utiliza sucessivas linearizações da função objetivo, obtendo subproblemas que são a determinação de centros analíticos de poliedros.

Exibiremos primeiramente algumas propriedades de funções convexas, a seguir abordaremos as idéias básicas encontradas em métodos de planos de corte e posteriormente passaremos à descrição do algoritmo de Goffin-Vial. 


\subsection{Propriedades de funções convexas}

Nesta seção demonstraremos alguns resultados sobre convexidade que serão úteis na definição do algoritmo.

Os principais resultados sobre $\varphi: \mathbb{R}^{n} \rightarrow \mathbb{R}$ convexa que nos interessam são:

- $\varphi$ é contínua

- $\varphi$ é subdiferenciável, isto é, existe um subgradiente em cada ponto

- Os subgradientes são localmente limitados

Definição 2.3 Uma função $\varphi: A \rightarrow \mathbb{R}, A \subset \mathbb{R}^{n}$ aberto, é subdiferenciável em $\bar{x}$ se existe $\gamma_{\bar{x}} \in \mathbb{R}^{n}$ tal que

$$
\varphi(x) \geq \varphi(\bar{x})+<\gamma_{\bar{x}}, x-\bar{x}>\quad \forall x \in A
$$

O vetor $\gamma_{\bar{x}}$ é chamado subgradiente de $\varphi(\cdot)$ em $\bar{x}$.

Definição 2.4 Dado $\gamma_{\bar{x}}$ um subgradiente de $\varphi(\cdot)$ em $\bar{x}$, um hiperplano suporte de $\varphi(\cdot)$ em $\bar{x}$ é definido por:

$$
H\left(\bar{x}, \gamma_{\bar{x}}\right)=\left\{(x, y): y=\varphi(\bar{x})+<\gamma_{\bar{x}}, x-\bar{x}>\right\}
$$

A figura 2.3 mostra hiperplanos suportes de duas funções, sendo que na segunda ilustra a existência de infinitos hiperplanos suportes num ponto. A existência de um único subgradiente num ponto é garantida se a função é diferenciável neste ponto, sendo que o subgradiente é o próprio gradiente. Estes resultados não serão aqui detalhados, porém podem ser vistos em Rockafellar [17]. 

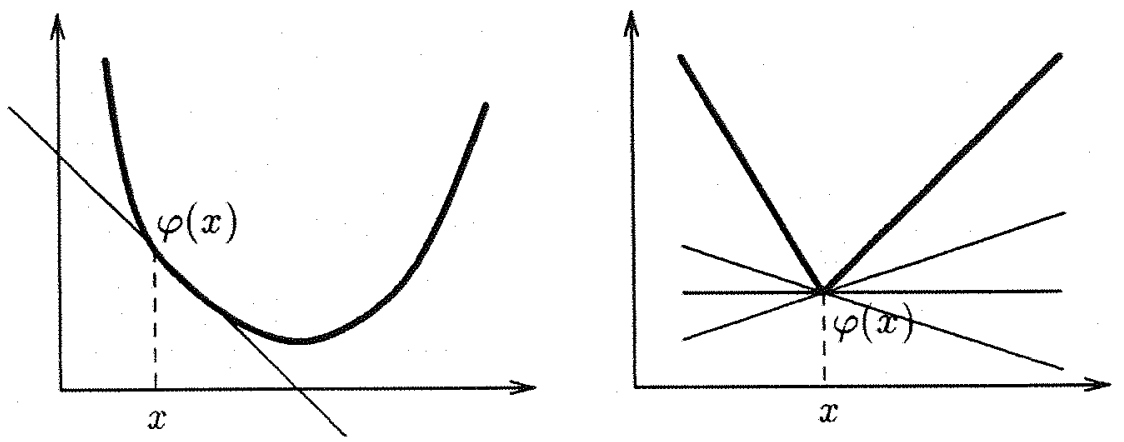

Figura 2.3: Hiperplano suporte de $\varphi$ em $x$

Definição 2.5 Um conjunto $X$ é dito convexo se para todo $x^{1}, x^{2} \in X$ e todo $\lambda \in[0,1]$ temos que

$$
\lambda x^{1}+(1-\lambda) x^{2} \in X
$$

ou seja, o segmento de reta que vai de $x^{1}$ a $x^{2}$ está contido em $X$.

Um fato interessante é que a epígrafe de uma função convexa é um conjunto convexo.

Proposição 2.1 Se $\varphi: X \rightarrow \mathbb{R}$ é convexa, com $X \subset \mathbb{R}^{n}$ convexo. então

$$
G_{\varphi}=\{(x, y) \in X \times \mathbb{R}: y \geq \varphi(x)\} \quad \text { é convexo }
$$

Demonstraçấo Sejam $\left(x^{1}, y^{1}\right),\left(x^{2}, y^{2}\right) \in G_{\varphi}, \lambda \in[0,1]$.

Como $X$ é convexo, $\lambda x^{1}+(1-\lambda) x^{2} \in X$.

Além disso, pela definição de $G_{\varphi}: \lambda y^{1}+(1-\lambda) y^{2} \geq \lambda \varphi\left(x^{1}\right)+(1-\lambda) \varphi\left(x^{2}\right)$ 
Como $\varphi$ é convexa: $\lambda \varphi\left(x^{1}\right)+(1-\lambda) \varphi\left(x^{2}\right) \geq \varphi\left(\lambda x^{1}+(1-\lambda) x^{2}\right)$

Portanto $\lambda y^{1}+(1-\lambda) y^{2} \geq \varphi\left(\lambda x^{1}+(1-\lambda) x^{2}\right)$

Logo $\lambda\left(x^{1}, y^{1}\right)+(1-\lambda)\left(x^{2}, y^{2}\right) \in G_{\varphi}$

Definição 2.6 Uma função $\varphi: \mathbb{R}^{n} \rightarrow \mathbb{R}$ é dita estável em $\bar{x} \in \mathbb{R}^{n}$ se existe um número real positivo $M_{\bar{x}}$ tal que

$$
\varphi(\bar{x})-\varphi(x)<M_{\bar{x}}\|\bar{x}-x\| \quad \forall x \in \mathbb{R}^{n}
$$

Definição 2.7 Uma função $\varphi: \mathbb{R}^{n} \rightarrow \mathbb{R}$ é dita localmente estável em $\bar{x} \in \mathbb{R}^{n}$ se existe um par de números reais positivos $\left(r, M_{\bar{x}, r}\right)$ tais que

$$
\varphi(\bar{x})-\varphi(x)<M_{\bar{x}, r}\|\bar{x}-x\| \quad \forall x \in B(\bar{x}, r)
$$

O teorema abaixo mostra um resultado mais forte que estabilidade local quando $\varphi(\cdot)$ é convexa.

Teorema 2.1 Seja $\varphi: \mathbb{R}^{n} \rightarrow \mathbb{R}$ convexa. Então $\forall \bar{x} \in \mathbb{R}^{n}, \forall r>0$, $\exists M_{\bar{x}, r}$ tal que:

$$
|\varphi(x)-\varphi(\bar{x})| \leq M_{\bar{x}, r}\|x-\bar{x}\| \quad \forall x \in B(\bar{x}, r)
$$

Demonstração

Consideremos a bola fechada, relativa à $\|\cdot\|_{\infty}$, de centro $\bar{x}$ e raio $r$ :

$$
B_{\infty}[\bar{x}, r]=\left\{\bar{x}+h:-r \leq h_{j} \leq r, \quad 1 \leq j \leq n\right\}
$$

$B_{\infty}[\bar{x}, r]$ é um hipercubo de centro $\bar{x}$ e lado $2 r$. Sejam $n v$ o número de vértices do hipercubo e $\left\{v_{i}\right\}_{1 \leq i \leq n v}$, o conjunto desses vértices. 
Então $B_{\infty}[\bar{x}, r]$ é o casco convexo de seus vértices $\left\{v_{i}\right\}_{1 \leq i \leq n v}$, ou seja: $\forall x \in B_{\infty}[\bar{x}, r], x$ pode ser escrito como $x=\sum_{i=1}^{n v} \lambda_{i} v_{i}$ com $0 \leq \lambda_{i} \leq 1$ e $\sum_{i=1}^{n v} \lambda_{i}=1$

Chamemos de $\bar{v}$ ao vértice de maior valor de $\varphi$ :

$$
\bar{v}=\underset{1 \leq i \leq n v}{\operatorname{argmax}}\left\{\varphi\left(v_{i}\right)\right\}
$$

Para $x \in B_{\infty}[\bar{x}, r]$, temos que

$$
\begin{array}{rlrl}
\varphi(x) & =\varphi\left(\sum_{i=1}^{n v} \lambda_{i} v_{i}\right) & \varphi \stackrel{\varphi}{\text { convexa }} \\
& \leq \sum_{i=1}^{n v} \lambda_{i} \varphi\left(v_{i}\right) & \stackrel{(i i)}{\leq} \\
& \leq \sum_{i=1}^{n v} \lambda_{i} \varphi(\bar{v}) & & = \\
& =\varphi(\bar{v}) \sum_{i=1}^{n v} \lambda_{i} & & \stackrel{(i)}{=} \\
& =\varphi(\bar{v}) &
\end{array}
$$

Portanto

$$
\varphi(x) \leq \varphi(\bar{v}) \quad \forall x \in B_{\infty}[\bar{x}, r] \quad(I)
$$

Por outro lado, $x \in B_{\infty}(\bar{x}, r), x \neq \bar{x}$, é da forma: $x=\bar{x}+h$ onde $0<\|h\|_{\infty}<r$. Tomemos $y=\bar{x}+h^{\prime}$ onde $h^{\prime}=\frac{r}{\|h\|_{\infty}} h$.

$\frac{\|h\|_{\infty}}{r} y+\left(1-\frac{\|h\|_{\infty}}{r}\right) \bar{x}=\frac{\|h\|_{\infty}}{r}\left(\bar{x}+\frac{r}{\|h\|_{\infty}} h\right)+\bar{x}-\frac{\|h\|_{\infty}}{r} \bar{x}=\bar{x}+h=x$

Portanto $x=\lambda y+(1-\lambda) \bar{x}$ onde $0<\lambda=\frac{\|h\|_{\infty}}{r}<1$

$$
\begin{array}{rlr}
\varphi(x) & =\varphi(\lambda y+(1-\lambda) \bar{x}) & \varphi \text { convex } \\
& \leq \lambda \varphi(y)+(1-\lambda) \varphi(\bar{x}) & \leq \\
& =\lambda(\varphi(y)-\varphi(\bar{x}))+\varphi(\bar{x}) &
\end{array}
$$


Reescrevendo:

$$
\varphi(x) \leq \frac{\|h\|_{\infty}}{r}(\varphi(y)-\varphi(\bar{x}))+\varphi(\bar{x})
$$

Por $(I)$

$$
\frac{\varphi(x)-\varphi(\bar{x})}{\|h\|_{\infty}} \leq \frac{\varphi(y)-\varphi(\bar{x})}{r} \leq \frac{\varphi(\bar{v})-\varphi(\bar{x})}{r}
$$

Chamando $M_{\bar{x}, r}=\frac{\varphi(\bar{v})-\varphi(\bar{x})}{r}$

$$
\frac{\varphi(x)-\varphi(\bar{x})}{\|x-\bar{x}\|_{\infty}} \leq M_{\bar{x}, r} \quad \forall x \in B_{\infty}[\bar{x}, r] \quad(I I)
$$

Como $\bar{x}=1 / 2(\bar{x}+h)+1 / 2(\bar{x}-h)$, segue que

$$
\varphi(\bar{x})=\varphi(1 / 2(\bar{x}+h)+1 / 2(\bar{x}-h)) \leq 1 / 2 \varphi(\bar{x}+h)+1 / 2 \varphi(\bar{x}-h)
$$

Como $2 \varphi(\bar{x}) \leq \varphi(\bar{x}+h)+\varphi(\bar{x}-h)$, por $(I I)$, temos que:

$$
\frac{\varphi(\bar{x})-\varphi(\bar{x}+h)}{\|h\|_{\infty}} \leq \frac{\varphi(\bar{x}-h)-\varphi(\bar{x})}{\|h\|_{\infty}} \leq M_{\bar{x}, r}
$$

Logo,

$$
\frac{\varphi(\bar{x})-\varphi(x)}{\|x-\bar{x}\|_{\infty}} \leq M_{\bar{x}, r} \quad(I I I)
$$

De $(I I)$ e $(I I I)$ concluimos que:

$$
|\varphi(x)-\varphi(\bar{x})| \leq M_{\bar{x}, r}\|x-\bar{x}\|_{\infty} \quad \forall x \in B_{\infty}[\bar{x}, r]
$$

Como $\|x-\bar{x}\|_{\infty} \leq\|x-\bar{x}\|$ e $B(\bar{x}, r) \subset B_{\infty}[\bar{x}, r]$,

$$
|\varphi(x)-\varphi(\bar{x})| \leq M_{\bar{x}, r}\|x-\bar{x}\| \quad \forall x \in B(\bar{x}, r)
$$


Corolário 2.1 Seja $\varphi: \mathbb{R}^{n} \rightarrow \mathbb{R}$ convexa. Então $\varphi(\cdot)$ é contínua.

Demonstração Seja $\bar{x} \in \mathbb{R}^{n}$ e $r>0$. Para todo $x \in B(\bar{x}, r)$, temos que $\exists M_{\bar{x}, r}$ com:

$$
0 \leq|\varphi(x)-\varphi(\bar{x})| \leq M_{\bar{x}, r}\|x-\bar{x}\|
$$

portanto

$$
0 \leq \lim _{x \rightarrow \bar{x}}|\varphi(x)-\varphi(\bar{x})| \leq \lim _{x \rightarrow \bar{x}} M_{\bar{x}, r}\|x-\bar{x}\|=0
$$

Logo: $\lim _{x \rightarrow \bar{x}} \varphi(x)=\varphi(\bar{x})$

Corolário 2.2 Seja $\varphi: \mathbb{R}^{n} \rightarrow \mathbb{R}$ convexa. Então $\varphi(\cdot)$ é estável, ou seja, $\forall \bar{x} \in \mathbb{R}^{n}, \exists M_{\bar{x}}$ tal que

$$
\varphi(\bar{x})-\varphi(x)<M_{\bar{x}}\|\bar{x}-x\| \quad \forall x \in \mathbb{R}^{n}
$$

Demonstração Pelo teorema 2.1, temos que para $\bar{x} \in \mathbb{R}^{n}, r>0, \bar{r}<r$

$$
\varphi(\bar{x})-\varphi(x) \leq M_{\bar{x}, \bar{r}}\|\bar{x}-x\| \quad \forall x \in B[\bar{x}, \bar{r}]
$$

Seja $x^{1} \in \mathbb{R}^{n}$ e $h^{1}=x^{1}-\bar{x}$. Se $\left\|h^{1}\right\| \leq \bar{r}$, por $(i)$, segue que $x^{1}$ satisfaz as condições da tese.

Analisemos então o caso em que $\left\|h^{1}\right\|>\bar{r}$.

Tomemos $x^{2}=\bar{x}+h^{2}$ onde $h^{2}=\frac{\bar{r}}{\left\|h^{1}\right\|} h^{1}$.

Note que $x^{2}$ é combinação convexa de $\bar{x}$ e $x^{1}$, pois

$x^{2}=\bar{x}+h^{2}=\bar{x}+\frac{\bar{r}}{\left\|h^{1}\right\|} h^{1}=\frac{\bar{r}}{\left\|h^{1}\right\|}\left(\bar{x}+h^{1}\right)+\frac{\left\|h^{1}\right\|-\bar{r}}{\left\|h^{1}\right\|} \bar{x}=\frac{\bar{r}}{\left\|h^{1}\right\|} x^{1}+\left(1-\frac{\bar{r}}{\left\|h^{1}\right\|}\right) \bar{x}$

$\operatorname{com} \frac{\bar{r}}{\left\|h^{1}\right\|}<1$. Pela convexidade de $\varphi(\cdot)$,

$\varphi\left(x^{2}\right)=\varphi\left(\frac{\bar{r}}{\left\|h^{1}\right\|} x^{1}+\left(1-\frac{\bar{r}}{\left\|h^{1}\right\|}\right) \bar{x}\right) \leq \frac{\bar{r}}{\left\|h^{1}\right\|} \varphi\left(x^{1}\right)+\left(1-\frac{\bar{r}}{\left\|h^{1}\right\|}\right) \varphi(\bar{x})$ 
Dai temos

$$
\left\|h^{1}\right\| \varphi\left(x^{2}\right) \leq \bar{r} \varphi\left(x^{1}\right)+\left(\left\|h^{1}\right\|-\bar{r}\right) \varphi(\bar{x})
$$

Portanto

$$
\bar{r}\left(\varphi(\bar{x})-\varphi\left(x^{1}\right)\right) \leq\left\|h^{1}\right\|\left(\varphi(\bar{x})-\varphi\left(x^{2}\right)\right)
$$

Por outro lado, pela hipótese: $\varphi(\bar{x})-\varphi\left(x^{2}\right) \leq M_{\bar{x}, \bar{r}}\left\|\bar{x}-x^{2}\right\|$ e por $\left\|h^{2}\right\|=\bar{r}$ temos que

$$
\varphi(\bar{x})-\varphi\left(x^{2}\right) \leq M_{\bar{x}, \bar{r}}\left\|h^{2}\right\|=M_{\bar{x}, \bar{r}} \bar{r}
$$

De (ii) e (iii) vem que

$$
\bar{r}\left(\varphi(\bar{x})-\varphi\left(x^{1}\right)\right) \leq\left\|h^{1}\right\|\left(\varphi(\bar{x})-\varphi\left(x^{2}\right)\right) \leq\left\|h^{1}\right\| M_{\bar{x}, \bar{r}} \bar{r}
$$

E como $\left\|h^{1}\right\|=\left\|\bar{x}-x^{1}\right\|$ vem que

$$
\varphi(\bar{x})-\varphi\left(x^{1}\right) \leq M_{\bar{x}, \bar{r}}\left\|\bar{x}-x^{1}\right\|
$$

Como $x^{\prime}$ foi tomado arbitrariamente e $\bar{r}$ não depende de $x^{1}$, temos que

$$
\varphi(\bar{x})-\varphi(x) \leq M_{\bar{x}, \bar{r}}\|\bar{x}-x\| \quad \forall x \in \mathbb{R}^{n}
$$

Utilizaremos um resultado clássico de convexidade, o teorema de Separação de Convexos, enunciado a seguir:

Teorema 2.2 Separação de Convexos.

Sejam $X$ e $Y$ dois conjuntos convexos disjuntos em $\mathbb{R}^{n}$. Então existe um plano $\left\{x: x \in \mathbb{R}^{n}, \alpha^{T} x=\beta\right\}$, com $\alpha \neq 0$, que os separa, ou seja,

$x \in X \Longrightarrow \alpha^{T} x \leq \beta$

$y \in Y \Longrightarrow \alpha^{T} y \geq \beta$

Além disso, se $X$ é aberto, temos que

$x \in X \Longrightarrow \alpha^{T} x<\beta$

$y \in Y \Longrightarrow \alpha^{T} y \geq \beta$ 
Demonstração Pode ser vista em Mangasarian [13].

Lema 2.1 Sejam $\varphi: \mathbb{R}^{n} \rightarrow \mathbb{R}$ convexa, $\bar{x} \in \mathbb{R}^{n}$ e $\bar{M}>0$. Então o conjunto $E=\left\{(x, y) \in \mathbb{R}^{n} \times \mathbb{R}: y<\varphi(\bar{x})-\bar{M}\|x-\bar{x}\|\right\}$ é convexo $e$ aberto.

\section{Demonstração}

\section{(a) $E$ é convexo}

Sejam $\left(x^{1}, y^{1}\right),\left(x^{2}, y^{2}\right) \in E$ e $\lambda \in[0,1]$.

Pela definição de $E$

$$
\begin{aligned}
& \lambda y^{1}<\lambda \varphi(\bar{x})-\lambda \bar{M}\left\|x^{1}-\bar{x}\right\| \\
& (1-\lambda) y^{2}<(1-\lambda) \varphi(\bar{x})-(1-\lambda) \bar{M}\left\|x^{2}-\bar{x}\right\|
\end{aligned}
$$

Portanto $\lambda y^{1}+(1-\lambda) y^{2}<\varphi(\bar{x})-\bar{M}\left(\lambda\left\|x^{1}-\bar{x}\right\|+(1-\lambda)\left\|x^{2}-\bar{x}\right\|\right)$

E como: $\lambda\left\|x^{1}-\bar{x}\right\|+(1-\lambda)\left\|x^{2}-\bar{x}\right\| \geq\left\|\lambda\left(x^{1}-\bar{x}\right)+(1-\lambda)\left(x^{2}-\bar{x}\right)\right\|$

temos que $\lambda y^{1}+(1-\lambda) y^{2}<\varphi(\bar{x})-\bar{M}\left\|\left(\lambda x^{1}+(1-\lambda) x^{2}\right)-\bar{x}\right\|$

Logo $\lambda\left(x^{1}, y^{1}\right)+(1-\lambda)\left(x^{2}, y^{2}\right) \in E$

(b) $E$ é aberto

Tomemos o complementar de $E$ em $\mathbb{R}^{n}$ :

$$
\bar{E}=\left\{(x, y) \in \mathbb{R}^{n} \times \mathbb{R}: y \geq \varphi(\bar{x})-\bar{M}\|x-\bar{x}\|\right\}
$$

Seja a sequência $\left\{\left(x^{k}, y^{k}\right)\right\} \rightarrow\left(x^{\prime}, y^{\prime}\right) \quad \operatorname{com}\left(x^{k}, y^{k}\right) \in \bar{E} \quad \forall k$.

Então

$$
\begin{aligned}
& y^{k} \geq \varphi(\bar{x})-\bar{M} \quad\left\|x^{k}-\bar{x}\right\| \quad \forall k \\
& \downarrow \\
& y^{\prime} \geq \varphi(\bar{x})-\bar{M} \quad\left\|x^{\prime}-\bar{x}\right\| \Longrightarrow\left(x^{\prime}, y^{\prime}\right) \in E
\end{aligned}
$$

Logo $\bar{E}$ é fechado, o que completa a demonstração. 
Teorema 2.3 Seja $\varphi: \mathbb{R}^{n} \rightarrow \mathbb{R}$ convexa.

Então $\varphi(\cdot)$ é subdiferenciável.

\section{Demonstração}

Seja $\bar{x} \in \mathbb{R}^{n}$. Pelo corolário $2.2, \varphi(\cdot)$ é estável em $\bar{x}$, ou seja, $\exists M_{\bar{x}}$ tal que

$$
\varphi(x) \geq \varphi(\bar{x})-\bar{M}_{\bar{x}}\|x-\bar{x}\|
$$

Tomemos o conjunto:

$$
E=\left\{(x, y) \in \mathbb{R}^{n} \times \mathbb{R}: \varphi(\bar{x})-\bar{M}_{\bar{x}}\|x-\bar{x}\|>y\right\}
$$

(a) $E$ é convexo e aberto, pelo lema anterior

(b) $G_{\varphi}$ é convexo, pois $\varphi$ é convexa

(c) $E \cap G_{\varphi}=\emptyset$, pois

$$
\begin{aligned}
& (x, y) \in E \Longleftrightarrow \varphi(\bar{x})-\bar{M}_{\bar{x}}\|x-\bar{x}\|>y \Longrightarrow y<\varphi(x) \\
& (x, y) \in G_{\varphi} \Longleftrightarrow y \geq \varphi(x)
\end{aligned}
$$

Por (a), (b) e (c), E e $G_{\varphi}$ satisfazem as hipóteses do teorema de separação de convexos, então $\exists\left(\begin{array}{c}\alpha \\ \alpha_{0}\end{array}\right) \neq 0$ tal que $\forall\left(x^{1}, y^{1}\right) \in G_{\varphi}, \forall\left(x^{2}, y^{2}\right) \in E$

$$
\alpha_{0} y^{1}+\alpha^{T} x^{1}>\alpha_{0} y^{2}+\alpha^{T} x^{2}
$$

Em particular, como $(\bar{x}, \varphi(\bar{x})+1) \in G_{\varphi}$ e $(\bar{x}, \varphi(\bar{x})-1) \in E$, substituindo em (ii), temos que

$\alpha_{0}(\varphi(\bar{x})+1)+\alpha^{T} \bar{x}>\alpha_{0}(\varphi(\bar{x})-1)+\alpha^{T} \bar{x} \Longrightarrow \alpha_{0}>-\alpha_{0} \Longrightarrow \alpha_{0}>0$ Portanto, tomando $\beta=\frac{1}{\alpha_{0}} \alpha$, (ii) pode ser rescrita como

$$
y^{1}+\beta^{T} x^{1}>y^{2}+\beta^{T} x^{2} \quad \forall\left(x^{1}, y^{1}\right) \in G_{\varphi}, \quad \forall\left(x^{2}, y^{2}\right) \in E
$$

Como $\forall x \in \mathbb{R}^{n}(x, \varphi(x)) \in G_{\varphi}$ e $\forall \varepsilon>0(\bar{x}, \varphi(\bar{x})-\varepsilon) \in E$, substituindo em (iii) temos que

$$
\varphi(x)+\beta^{T} x>\varphi(\bar{x})-\varepsilon+\beta^{T} \bar{x} \quad \forall x \in \mathbb{R}^{n}, \quad \forall \varepsilon>0
$$


Tomando o supremo para $\varepsilon>0$,

$$
\varphi(x)+\beta^{T} x \geq \varphi(\bar{x})+\beta^{T} \bar{x} \quad \forall x \in \mathbb{R}^{n}
$$

Portanto

$$
\varphi(x) \geq \varphi(\bar{x})+<-\beta, x-\bar{x}>\quad \forall x \in \mathbb{R}^{n}
$$

Logo $\varphi(\cdot)$ é subdiferenciável em $\bar{x}$.

Dentro das hipóteses de $\varphi: \mathbb{R}^{n} \rightarrow \mathbb{R}$ convexa e $S$ compacto, o teorema a seguir mostra que o subgradiente é globalmente limitado.

Teorema 2.4 Seja $\varphi: \mathbb{R}^{n} \rightarrow \mathbb{R}$ convexa, $\bar{x} \in \mathbb{R}^{n}, r>0$ e $S$ compacto. Então existe $G \in \mathbb{R}$ tal que: para todo $x \in S$ e $\gamma_{x}$, subgradiente de $\varphi$ em $x$, temos que $\left\|\gamma_{x}\right\| \leq G$.

\section{Demonstração}

Pelo teorema 2.3, $\varphi$ é diferenciável em todos os pontos. Seja $x \in S \subset \mathbb{R}^{n}$ e $\gamma_{x}$ um subgradiente de $\varphi(\cdot)$ em $x$.

Se $\gamma_{x}=0$, nada a ser provado. Caso contrário, tomemos $h_{x}=\frac{\gamma_{x}}{\left\|\gamma_{x}\right\|}$. Pelo teorema 2.3, temos que:

$$
\varphi\left(x+h_{x}\right) \geq \varphi(x)+<\gamma_{x}, h_{x}>
$$

e daí

$$
\varphi\left(x+h_{x}\right)-\varphi(x) \geq<\gamma_{x}, h_{x}>=<\gamma_{x}, \frac{\gamma_{x}}{\left\|\gamma_{x}\right\|}>=\left\|\gamma_{x}\right\|
$$

Consideremos agora um hipercubo $H_{L}$ de lado $2 L$, com $L$ suficientemente grande para conter o conjunto $\left\{z \in \mathbb{R}^{n}: z=y+h\right.$, com $y \in S$ e $\left.\|h\| \leq 1\right\}$. Notemos que, pelo corolário $2.1, \varphi(\cdot)$ é contínua, $\log 0 \varphi(\cdot)$ assume máximo e mínimo em qualquer compacto de $\mathbb{R}^{n}$. Chamemos então de $M$ e $m$, respectivamente, ao máximo e ao mínimo de $\varphi$ em $H_{L}$ :

$$
M=\max _{y \in H_{L}} \varphi(y) \quad m=\min _{y \in H_{L}} \varphi(y)
$$


Pela definição de $H_{L}$ e como $x \in S$ e $\left\|h_{x}\right\|=1$, temos que $x \in H_{L}$ e $x+h_{x} \in H_{L}$. Portanto,

$$
M \geq \varphi\left(x+h_{x}\right) \quad \text { e } \quad m \leq \varphi(x)
$$

Substituindo (2) em (1), vem que:

$$
M-m \geq \varphi\left(x+h_{x}\right)-\varphi(x) \geq\left\|\gamma_{x}\right\|
$$

Ou seja:

$$
\forall x \in S \quad\left\|\gamma_{x}\right\| \leq M-m \in \mathbb{R}
$$

\subsection{Algoritmos de Planos de Corte}

Nesta seção apresentaremos uma formulação geral para algoritmos de planos de corte (Eaves e Zangwill [3]), e na próxima seção aplicaremos algumas das idéias vistas ao algoritmo de Goffin-Vial.

Inicialmente definiremos uma funçâo chamada separador. Separadores são úteis quando tratamos de algoritmos de planos de corte pois, além de facilitarem a demonstração de convergência, são ferramentas teóricas para fazermos eliminaçôes de restrições.

Definição 2.8 Dados conjuntos fechados $X \supset G \neq \emptyset \mathrm{em} \mathbb{R}^{n}$, uma função $\delta: X \backslash G \rightarrow \mathbb{R}$ é um separador se as seguintes condições são satisfeitas:

a) $\delta$ é não negativa

b) Se $\lim _{k \rightarrow \infty} x^{k}=\bar{x} \in \lim _{k \rightarrow \infty} \delta\left(x^{k}\right)=0$ então $\bar{x} \in G$

Uma consequência da definição é que um separador deve ser estritamente positivo em $X \backslash G$.

Um exemplo de separador é a distância de um ponto $x \in X$ ao conjunto $G$, que chamamos de $d(x, G)$. 


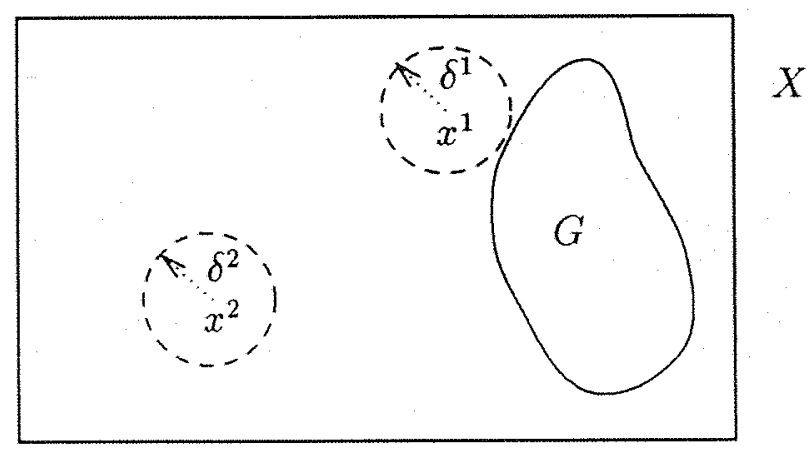

Figura 2.4: Exemplo de separador $\left(\delta^{k}=\delta\left(x^{k}\right)\right)$

São separadores:

- mínimo de uma coleção finita de separadores

- múltiplo positivo de separador

- função maior ou igual a um separador

A seguir temos um algoritmo que sintetiza os métodos de planos de corte. $\mathrm{O}$ algoritmo gera pontos em $X$ e pára quando $G$ é atingido.

\section{ALGORITMO BÁSICO DE PLANOS DE CORTE (ABPC)}

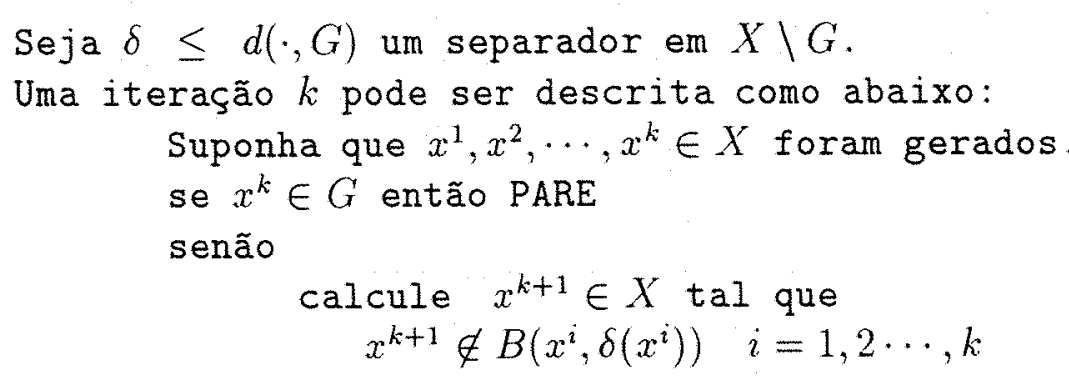




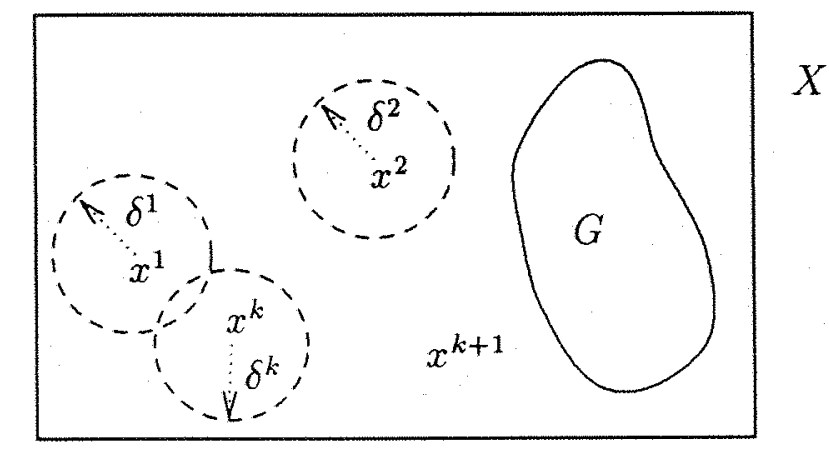

Figura 2.5: Exemplo de pontos gerados pelo algoritmo (ABPC)

Teorema 2.5 Sejam dois conjuntos fechados $X \supset G=\emptyset$, e seja a sequência $x^{1}, x^{2}, \cdots$ em $X$ gerada pelo algoritmo ( $\left.A B P C\right)$. Então qualquer ponto de acumulação da sequência está em $G$.

Demonstração Seja um ponto de acumulação $\bar{x}$ do algoritmo, ou seja, suponha para alguma subsequência $\bar{K}$ :

$$
\left\{x^{k}\right\}_{k \in \bar{K}} \longrightarrow \bar{x}
$$

Renumerando os indices de $\bar{K}$ de modo a $\bar{K}=\mathbb{N}$, temos que $\lim _{k \rightarrow \infty} x^{k}=\bar{x}$.

Pela construção do algoritmo temos que: $x^{k+1} \notin B\left(x^{i}, \delta\left(x^{i}\right)\right), i<k+1$. Portanto

$$
\left\|x^{k+1}-x^{k}\right\| \geq \delta\left(x^{k}\right) \geq 0 \quad \forall k
$$

Logo

$$
0=\lim _{k \rightarrow \infty}\left\|x^{k+1}-x^{k}\right\| \geq \lim _{k \rightarrow \infty} \delta\left(x^{k}\right) \geq 0
$$

Daí: $\lim _{x \rightarrow \infty} \delta\left(x^{k}\right)=0$ e pela definição de separador $\bar{x} \in G \quad \square$. 
Suponhamos que $X$ e $G$ são compactos. Vamos definir $\mathcal{Z}$ como a coleção de todos os conjuntos fechados $Z$ tais que $G \subset Z \subset X$.

$$
\mathcal{Z}=\{Z: G \subset Z \subset X, Z \text { fechado }\}
$$

Os conjuntos $Z \in \mathcal{Z}$ são chamados cortes.

Agora ligaremos os conceitos de separador e cortes definindo um mapa de corte. Lembremos que um mapa ponto-a-conjunto de $X \backslash G \rightarrow \mathcal{Z}$ leva pontos de $X \backslash G$ em subconjuntos de $\mathcal{Z}$

Definição 2.9 Um mapa ponto-a-conjunto $\mathcal{M}: X \backslash G \rightarrow \mathcal{Z}$ é um mapa de corte se existe um separador $\delta$ tal que para cada $x \in X \backslash G$ e cada $Z \in \mathcal{M}(x)$

$$
Z \cap B(x, \delta(x))=\emptyset
$$

Podemos escrever então um algoritmo de planos de corte a partir da geração de cortes. Na prática, os cortes $Z$ serão semi-espaços do $\mathbb{R}^{n}$.

\section{ALGORITMO MESTRE DE PLANOS DE CORTE (AMPC)}

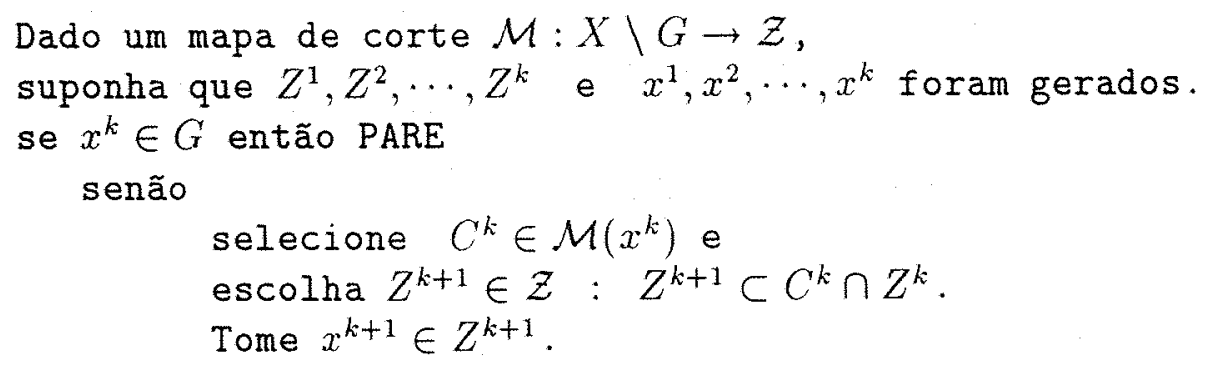

Teorema 2.6 Sejam $X$ e $G$ compactos com $\emptyset \neq G \subset X \subset \mathbb{R}^{n}$. Então a sequência de pontos $x^{1}, x^{2}, \cdots$ em $X$ gerada pelo algoritmo (AMPC) tem subsequência convergente com ponto de acumulaçâo em $G$. 
Demonstração Como a sequência de pontos $x^{1}, x^{2}, \cdots$ gerada pelo algoritmo (AMPC) está em $X$ compacto, existe subsequência convergente.

Seja $\left\{x^{k}\right\}_{k \in K} \longrightarrow \bar{x}$ uma tal subsequência e renumerando os índices de forma que $K=\mathbb{N}$, temos que $\lim _{k \rightarrow \infty} x^{k}=\bar{x}$.

O algoritmo (AMPC) gera conjuntos $Z^{k+1} \subset Z^{k} \cap C^{k} \subset Z^{k} \subset \cdots \subset Z^{i} \cap C^{i}$, $i=1, \ldots, k$, onde $C^{i} \in \mathcal{M}\left(x^{i}\right)$. Como $x^{k+1} \in Z^{k+1}$, então $x^{k+1} \in C^{i} \neq \emptyset$ e pela definição de mapa de corte:

$$
x^{k+1} \notin B\left(x^{i}, \delta\left(x^{i}\right)\right), \quad i<k+1
$$

Logo o algoritmo opera como (ABPC) e, pelo teorema $2.5, \bar{x} \in G$

\subsection{Descrição do algoritmo de Goffin-Vial}

Inicialmente descreveremos o algoritmo para o caso particular de quando a função objetivo é linear, e a seguir apresentaremos o algoritmo para qualquer função convexa em $\mathbb{R}^{n}$.

\subsubsection{Algoritmo para o caso linear}

Vamos inicialmente examinar o caso em que $\varphi(\cdot)$ é linear, procurando mostrar como o algoritmo pode ser visto como uma generalização de um particular algoritmo de trajetória central para programação linear, no sentido de que, aplicado ao caso linear, ele é um algoritmo de trajetória central.

Olhemos para o problema transformado para considerar pontos na epígrafe:

$$
\left(P L^{\prime}\right) \quad\left\{\begin{array}{l}
\min y \\
(x, y) \in G_{\varphi}=\left\{(x, y): A x \leq b, y \geq \varphi(x)=c^{T} x\right\}
\end{array}\right.
$$


Reescrevendo, temos:

$$
\left(P L^{\prime}\right) \quad\left\{\begin{array}{l}
\min y \\
A x+0 y \leq b \\
c^{T} x-y \leq 0
\end{array}\right.
$$

Podemos escrever o seguinte algoritmo para resolver o problema:

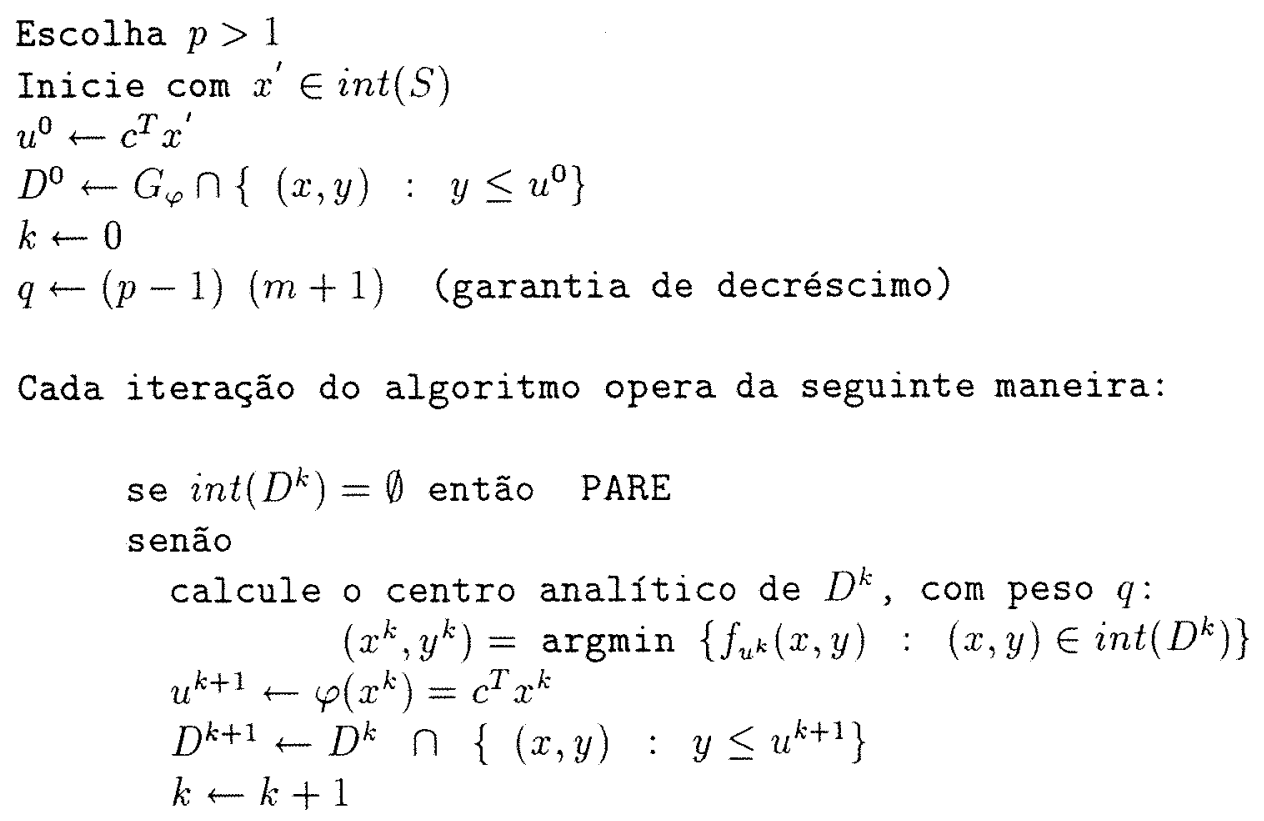

A figura 2.6 mostra como o conjunto inicial $D^{0}$ é obtido. A figura 2.7 mostra uma iteração típica. 


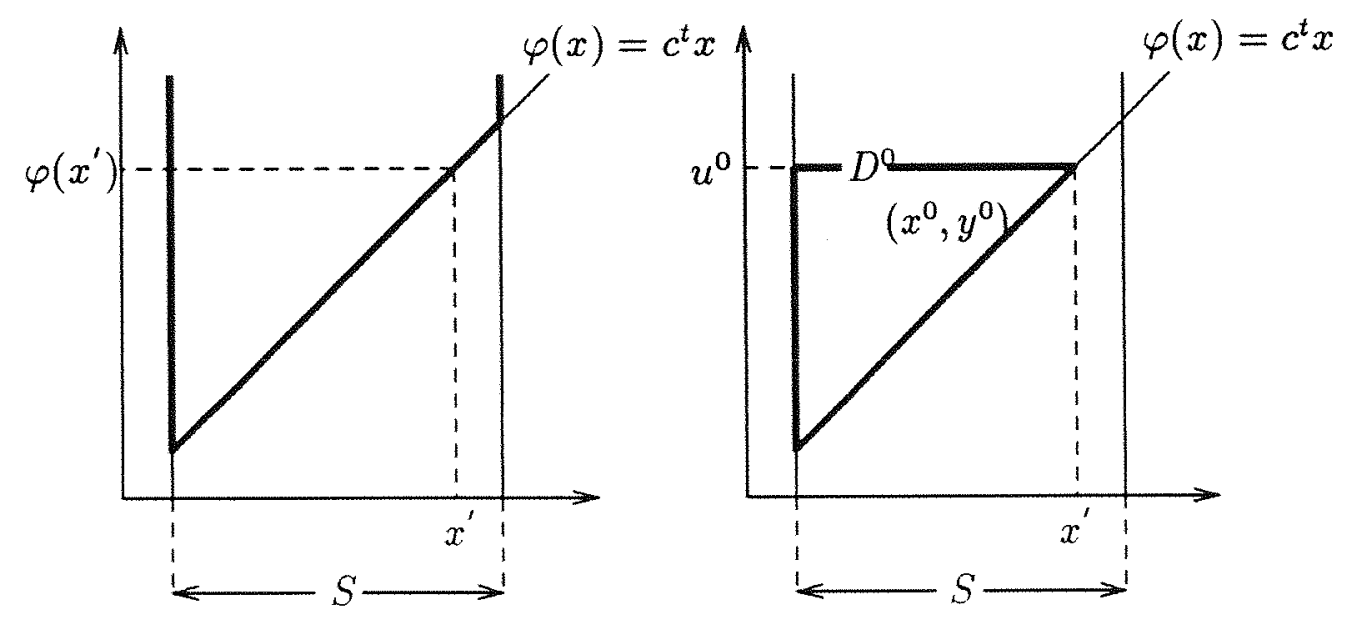

Figura 2.6: Obtenção do conjunto inicial $D^{0}$

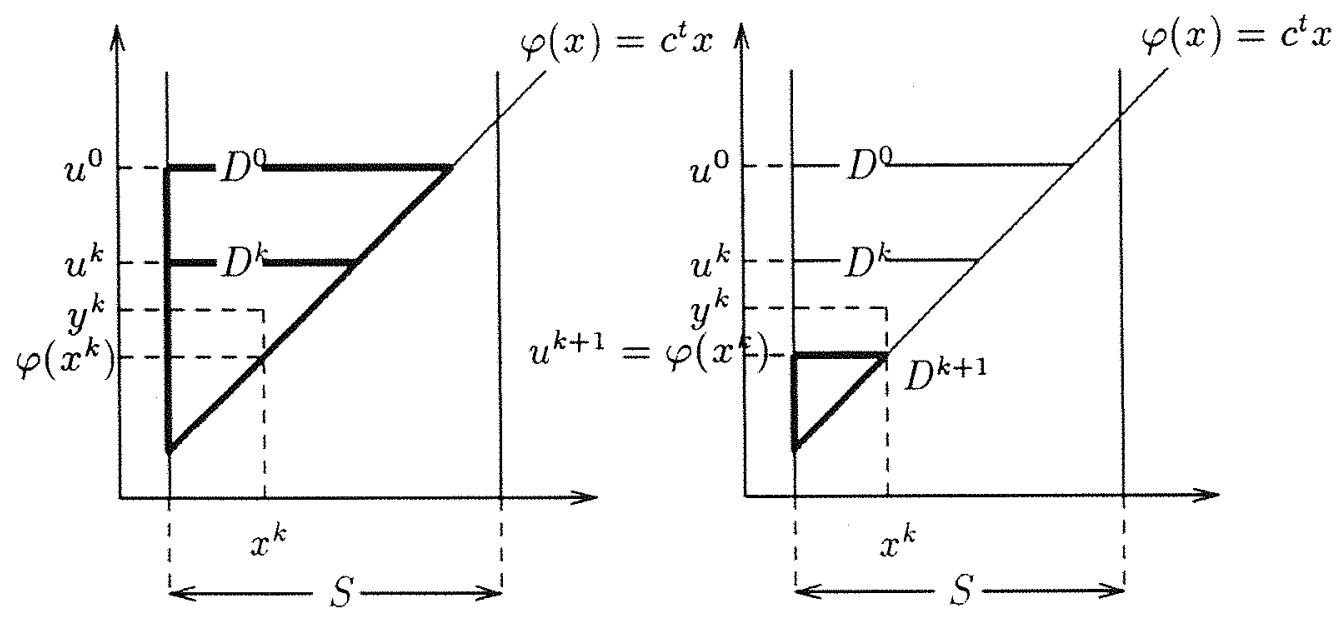

Figura 2.7: Iteração típica para $\varphi$ linear 
Proposição 2.2 O algoritmo descrito anteriormente gera uma sequência de pontos $x^{0}, x^{1}, x^{2}, \cdots$ tal que: $\varphi\left(x^{k}\right)<\varphi\left(x^{k-1}\right)+\frac{\varphi\left(x^{k-1}\right)-\varphi^{*}}{p}$ onde $\varphi^{*}$ é o valor ótimo de $\left(P L^{\prime}\right), \quad p>1$.

\section{Demonstração}

Seja $\left(x^{k}, y^{k}\right)$ o centro analítico de $D^{k}$. Pelo corolário 1.1, $y^{k} \leq u^{k}+\frac{u^{k}-\varphi^{*}}{p}$. Como $\left(x^{k}, y^{k}\right)$ é interior à epígrafe de $\varphi, \varphi\left(x^{k}\right)<y^{k} \leq u^{k}+\frac{u^{k}-\varphi^{*}}{p}$.

Por outro lado, $u^{k}=\varphi\left(x^{k-1}\right)$ pela definição do algoritmo, então :

$$
\varphi\left(x^{k}\right)<y^{k} \leq u^{k}+\frac{u^{k}-\varphi^{*}}{p}=\varphi\left(x^{k-1}\right)+\frac{\varphi\left(x^{k-1}\right)-\varphi^{*}}{p}
$$

Este algoritmo pode ser visto apenas como um algoritmo teórico já que estamos calculando exatamente o centro analítico. Mais tarde esta suposição será aliviada.

\subsubsection{Algoritmo para o caso convexo}

Vamos examinar algumas idéias utilizadas no caso linear para generalizarmos quando $\varphi(\cdot)$ é convexa.

No caso linear, o conjunto inicial $D^{0}$ é obtido a partir da epígrafe de $\varphi$ em $S$, acrescentando uma restrição de limitante superior. Como $\varphi$ é linear, este é um conjunto descrito apenas por restriçôes lineares, permitindo a determinação do primeiro centro analítico. Relembrando,

$$
D^{0} \leftarrow G_{\varphi} \cap\left\{(x, y): y \leq u^{0}\right\}
$$

A seguir os conjuntos $D^{k}$ subsequentes são obtidos apenas atuculizando o limitante superior $u^{k} \leq u^{k+1}$

$$
D^{k} \leftarrow D^{k-1} \cap\left\{(x, y): y \leq u^{k}\right\}=G_{\varphi} \cap\left\{(x, y): y \leq u^{k}\right\}
$$

No caso de $\varphi(\cdot)$ convexa no $\mathbb{R}^{n}$, a cada passo, não só atualizaremos a restrição de limitante superior, como também acrescentaremos uma restrição de linearização de $\varphi$ no ponto obtido. Esta restrição de linearização é um semiespaço definido pelo hiperplano suporte de $\varphi$ num ponto, como explicitado abaixo: 


$$
L\left(\bar{x}, \gamma_{\bar{x}}\right)=\left\{(x, y) \in S \times \mathbb{R}: y \geq \varphi(\bar{x})+<\gamma_{\bar{x}}, x-\bar{x}>\right\}
$$

Consideremos inicialmente a epígrafe de $\varphi$ e $x^{\prime} \in \operatorname{int}(S)$.

Escolhemos $\gamma^{\prime}$ um subgradiente de $\varphi$ determinando $L\left(x^{\prime}, \gamma^{\prime}\right)$. O conjunto inicial $D^{0}$ é definido por

$$
D^{0} \leftarrow L\left(x^{\prime}, \gamma^{\prime}\right) \cap\left\{(x, y) \in S \times \mathbb{R}: y \leq u^{0}=\varphi\left(x^{\prime}\right)\right\}
$$
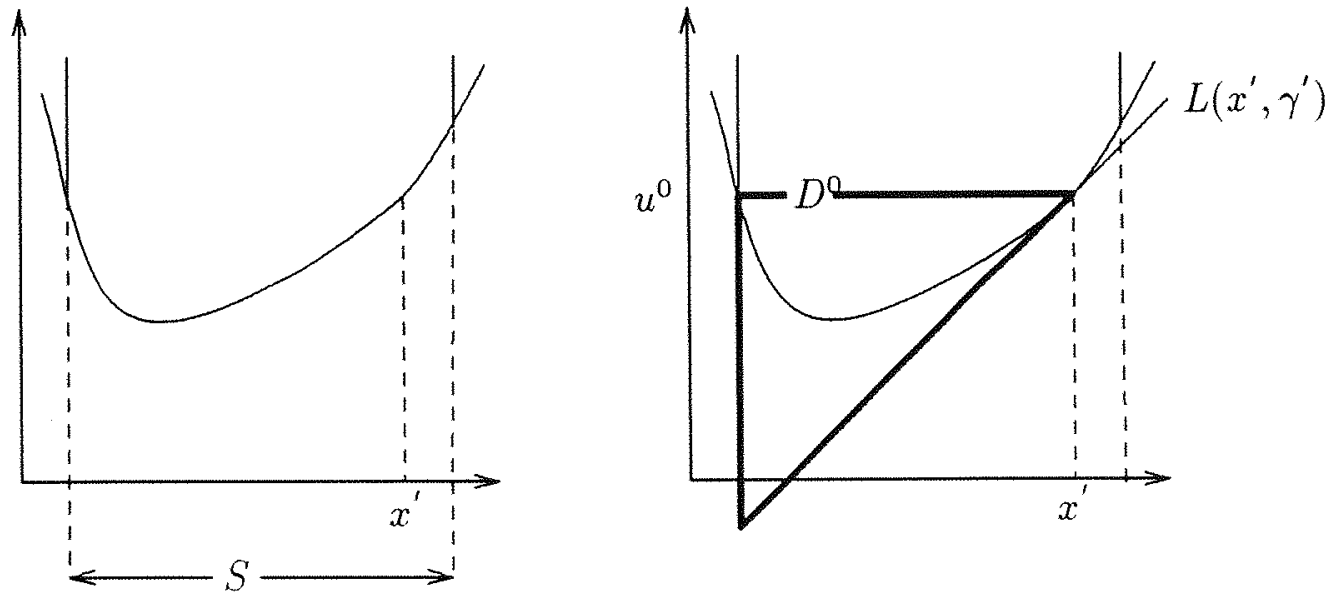

Figura 2.8: Obtenção do conjunto inicial $D^{0}$

Numa iteração $k$, calcula-se $\left(x^{k}, y^{k}\right)$ o centro analítico de $D^{k}$, escolhe-se um subgradiente $\gamma^{k}$ de $\varphi$ em $x^{k}$, acrescentando a restrição $L\left(x^{k}, \gamma^{k}\right)$ e atualizando-se a restrição de limitante superior com um novo $u^{k}$. 

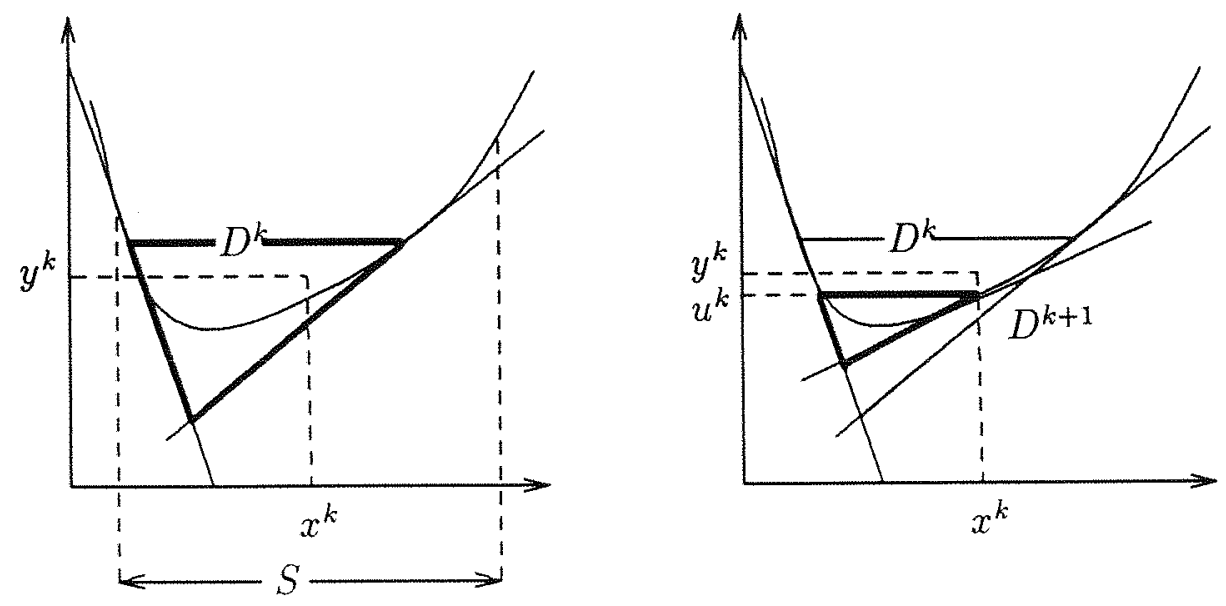

Figura 2.9: Iteração típica para $\varphi$ convexa
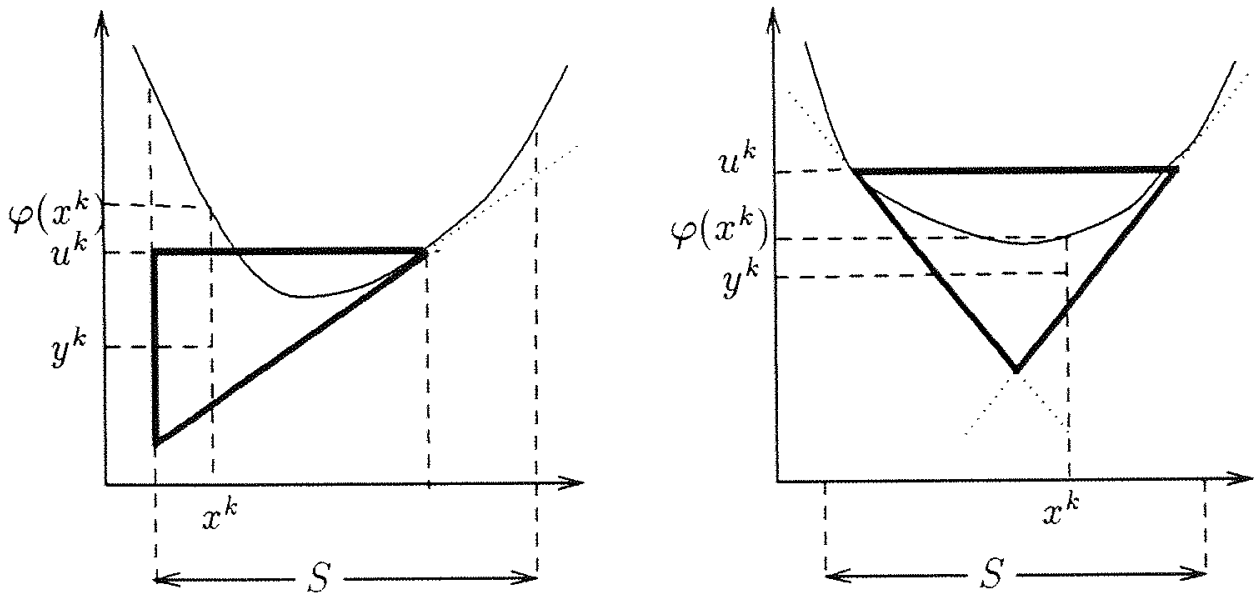

Figura 2.10: Diferenças na atualização de $u^{k}$ 
Vemos na figura 2.10 uma diferença fundamental com o caso linear: nem sempre o limitante superior, $u^{k}$, abaixa de uma iteração para outra. No primeiro caso haverá atualização de limitante superior e no segundo caso não pois $\varphi\left(x^{k}\right)>u^{k}$.

Outra diferença importante é que, quando $\varphi$ é linear, para todo centro $\left(x^{k}, y^{k}\right)$ de $D^{k}$, vale que $y^{k}>\varphi\left(x^{k}\right)$. As figuras abaixo mostram que isto nem sempre ocorre no caso de $\varphi$ convexa:
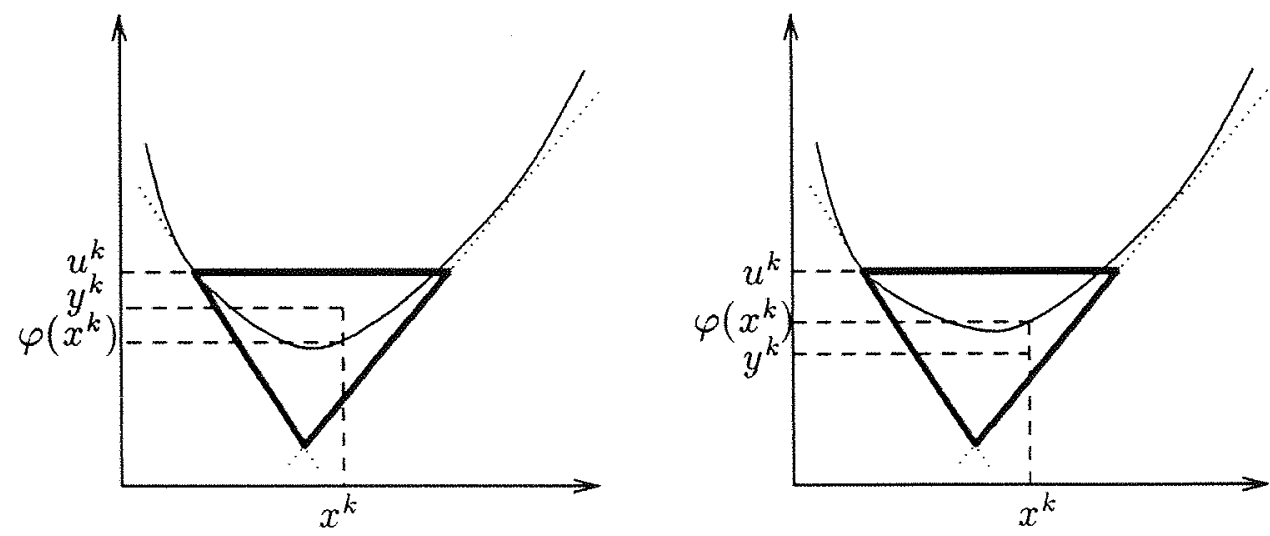

Figura 2.11: Posições de $y^{k}$ em relação à epígrafe

\section{Notação :}

$X^{*}=\left\{(x, y) \in S \times \mathbb{R}:(x, y)=\underset{(x, y) \in G_{\varphi}}{\operatorname{argmin}} y\right\} \quad$ (conjunto das soluções ótimas do problema).

$L(\bar{x}, \bar{\gamma})=\{(x, y) \in S \times \mathbb{R}: y \geq \varphi(\bar{x})+<\bar{\gamma}, x-\bar{x}>\}$, para $\bar{x} \in S \mathrm{e}$ $\bar{\gamma}$ um subgradiente de $\varphi$ em $\bar{x}(L(\bar{x}, \bar{\gamma})$ semiespaço definido pelo hiperplano suporte de $\varphi \mathrm{em} \bar{x}$ ).

$\mathcal{U}(u)=\{(x, y): y \leq u\}$, para $u \in \mathbb{R}$, limitante superior do valor ótimo de $\left(P^{\prime}\right)$. 


\section{Algoritmo de Goffin-Vial}

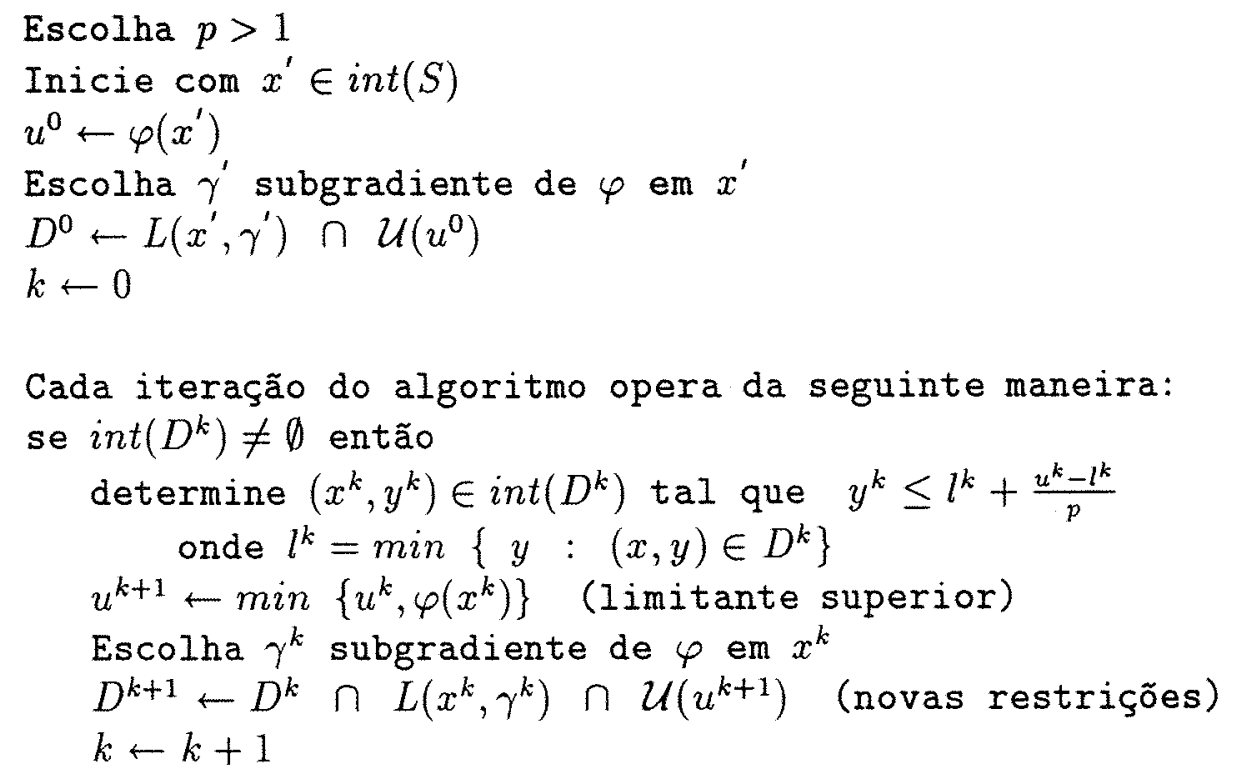

O algoritmo pára quando $\left(x^{k}, y^{k}\right)$ é tal que $\varphi\left(x^{k}\right)$ está suficientemente próximo do valor ótimo de ( $\left.\mathrm{P}^{\prime}\right)$ ou quando $\operatorname{int}\left(D^{k}\right)=\emptyset$ o que só ocorre se $\gamma^{k-1}=0$, implicando que $\left(x^{k-1}, y^{k-1}\right)$ é solução ótima de (P').

O algoritmo não está totalmente especificado já que não indicamos como resolver o subproblema.

\section{Solução do Subproblema}

"Determinar $\left(x^{k}, y^{k}\right) \in \operatorname{int}\left(D^{k}\right)$ tal que $y^{k} \leq l^{k}+\frac{u^{k}-l^{k}}{p}$, onde $l^{k}=\min \left\{y:(x, y) \in D^{k}\right\}$ e $p>1 "$.

O subproblema será resolvido calculando um centro analítico aproximado $\bar{y}$ do poliedro $D^{k}$ com função centro

$$
f_{u^{k}}(x, y)=-q \ln \left(u^{k}-y\right)-\sum_{i=1}^{m} \ln \left(\bar{b}_{i}-\bar{a}_{i}(x, y)\right)
$$


onde $\bar{A}$ e $\bar{b}$ formam as restrições $\bar{A}(x, y) \leq \bar{b}$ que definem o poliedro $D^{k}$, e $q$ é escolhido como no teorema 1.2 do capítulo anterior. Parando o algoritmo de centros com uma aproximação $(\bar{x}, \bar{y})$ tal que $\delta((\bar{x}, \bar{y}), u)$ satisfaz as condições daquele teorema, segue pela tese do teorema que $\bar{y}$ resolve o subproblema.

Note que aqui deve haver um ajuste de notações, observando que $l^{k}$ é valor ótimo do problema linear com função objetivo $c^{T}(x, y)=y$.

\subsubsection{Convergência}

Provaremos que a sequência de pontos gerada pelo algoritmo converge para a solução ótima de $\left(P C^{\prime}\right)$.

A convergência do algoritmo se baseia em duas idéias básicas:

- Os hiperplanos suporte que definem os cortes $L\left(x^{k}, \gamma^{k}\right)$, garantem uma convergência para pontos na epígrafe de $\varphi$.

- Existe uma sequência de limitantes superiores do valor ótimo, os $u^{k}$ gerados pelo algoritmo, e uma sequência implícita de limitantes inferiores $l^{k}$, valores ótimos dos subproblemas que convergem para um mesmo ponto.

Inicialmente, vamos a examinar algumas propriedades do algoritmo.

Proposição 2.3 Sejam $\left\{u^{k}\right\}$ e $\left\{\left(x^{k}, y^{k}\right)\right\}$ as sequências geradus pelo algoritmo de Goffin-Vial e $\varphi^{*}$ valor ótimo de $\left(P^{\prime}\right)$. Então:

$$
y^{i} \leq \varphi^{*}+\frac{u^{k}-\varphi^{*}}{p} \quad \forall i>k
$$

Demonstração Pela escolha de $\left(x^{i}, y^{i}\right)$ temos que

$$
y^{i} \leq l^{i}+\frac{u^{i}-l^{i}}{p}=\frac{(p-1) l^{i}+u^{i}}{p}
$$


Pela escolha de $u^{k}$ temos $u^{i} \leq u^{k} \quad \forall i>k$

Pela definição de $l^{i}$, como valor ótimo do problema linearizado, $l^{i} \leq$ $\varphi^{*}(I I I)$.

Substituindo $(I I)$ e $(I I I)$ em $(I)$, e sabendo que $p>1$, temos:

$$
y^{i} \leq \frac{(p-1) l^{i}+u^{i}}{p} \leq \frac{(p-1) \varphi^{*}+u^{k}}{p}=\varphi^{*}+\frac{u^{k}-\varphi^{*}}{p} \quad \forall i>k
$$

A proposição anterior nos mostra que, ao introduzir a restrição $y \leq u^{k}$ na definição de $\mathcal{U}\left(u^{k}\right)$, estamos acrescentando uma restrição implícita que garante:

$$
y^{i} \leq \varphi^{*}+\frac{u^{k}-\varphi^{*}}{p} \leq u^{k}
$$

O desenho abaixo ilustra essa afirmação para $p=2$.

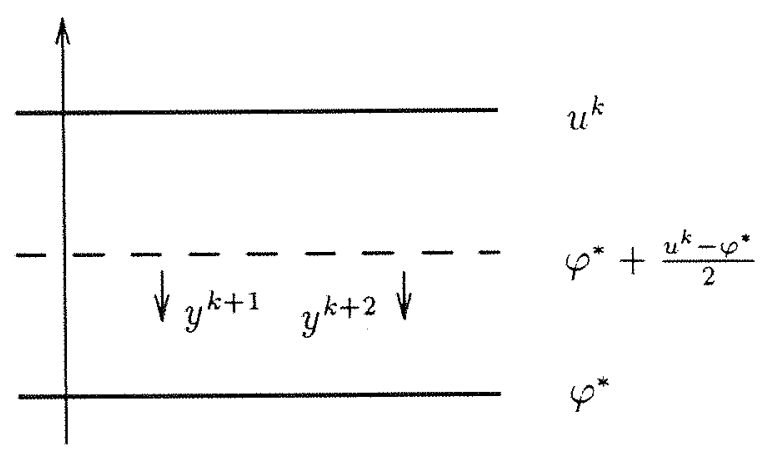

Figura 2.12: Restrição implícita relativa a $u^{k}$ 
Reescreveremos o algoritmo de Goffin-Vial de modo a facilitar a definição de um separador que o caracterize como um método de planos de corte.

Este é apenas um algoritmo teórico já que supõe o conhecimento do valor ótimo $\varphi^{*}$.

Nesta definição, são gerados uma série de cortes a partir de um conjunto inicial $X$ e o algoritmo pára quando $X^{*}$, conjunto das soluçóes ótimas do problema, é atingido.

\section{Algoritmo de Goffin-Vial como Planos de Corte (AGPC)}

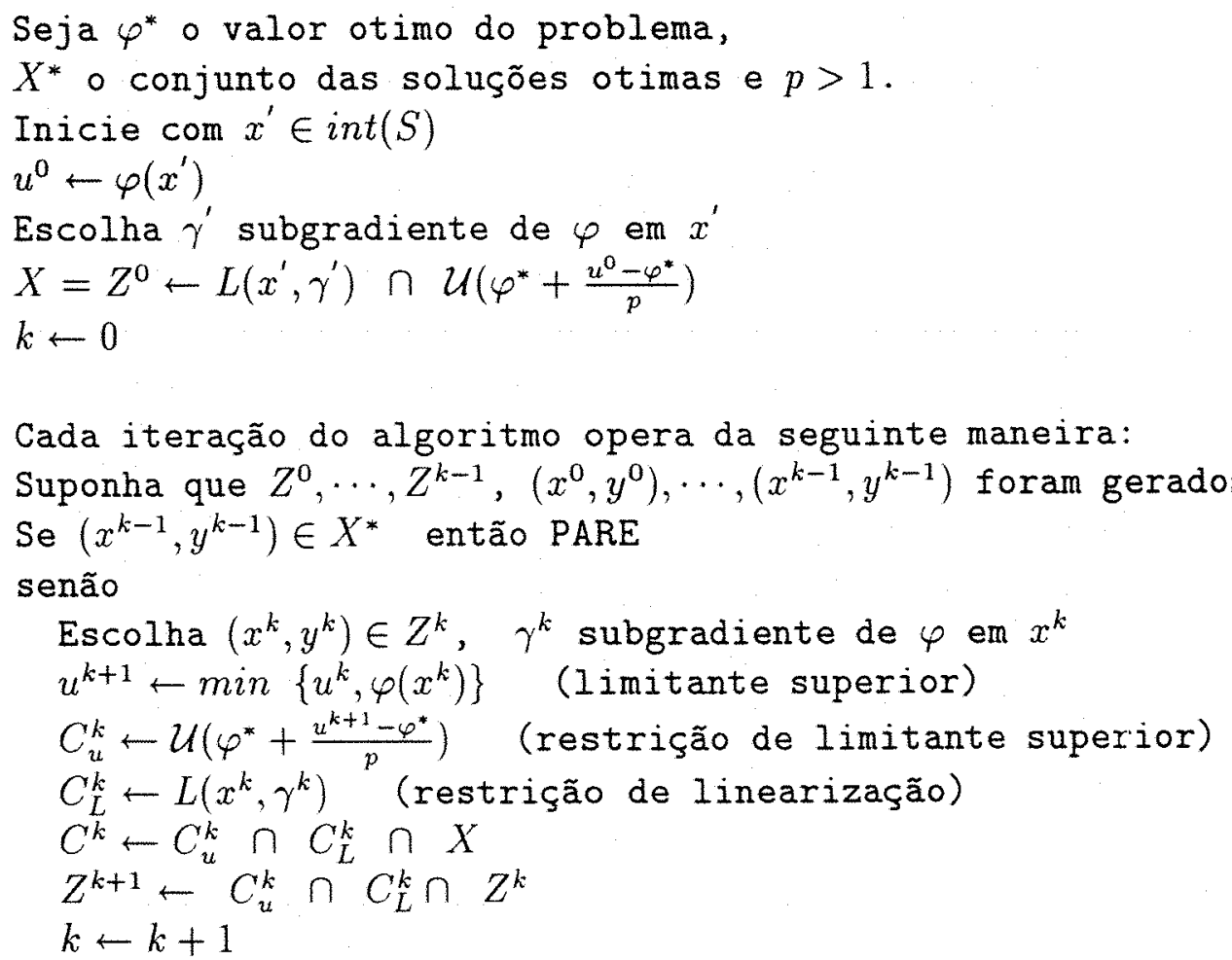

A diferença entre o algorimo acima e o original está na definição explícita de $C_{u}^{k}$ como corte de limitante superior. A proposição 2.3 mostrou que isto 
pode ser feito sem alterar os pontos gerados pelo algoritmo.

É fácil ver que $X$ é compacto, portanto para mostrarmos que (AGPC) opera como o algoritmo mestre de plano de corte (AMPC) da seção 2.2, resta mostrarmos que cada $C^{k}$ é um corte.

Para tanto, definiremos a função abaixo que mostraremos ser um separador de $X^{*}$.

$\delta: X \backslash X^{*} \rightarrow \mathbb{R}$, dada por: $\delta(x, y)=\max \{D(x, y), E(x, y)\}$ onde

$$
\begin{aligned}
& D(x, y)=\operatorname{dist}\left((x, y), L\left(x, \gamma_{x}\right)\right) \text { para } \gamma_{x} \text { subgradiente de } \varphi \text { em } x \\
& E(x, y)=(y-\varphi(x))+\frac{(p-1) \varphi(x)-\varphi^{*}}{p} \text { para } \varphi^{*} \text { valor ótimo de }\left(P^{\prime}\right) \\
& \text { e } p>1
\end{aligned}
$$

Lema 2.2 Se $\left\{\left(x^{k}, y^{k}\right)\right\} \rightarrow(\bar{x}, \bar{y}), D\left(x^{k}, y^{k}\right) \rightarrow 0$ e $y^{k} \leq \varphi\left(x^{k}\right)$, então $\bar{y}=\varphi(\bar{x})$.

Demonstraçãao $D\left(x^{k}, y^{k}\right)=\operatorname{dist}\left(\left(x^{k}, y^{k}\right), L\left(x^{k}, \gamma^{k}\right)\right)$ onde

$$
L\left(x^{k}, \gamma^{k}\right)=\left\{(x, y): y \geq \varphi\left(x^{k}\right)+<\gamma^{k}, x-x^{k}>\right\}
$$

Como $y^{k} \leq \varphi\left(x^{k}\right)$, temos que $y^{k} \leq \varphi\left(x^{k}\right)+<\gamma^{k}, x^{k}-x^{k}>$ $\Longrightarrow\left(x^{k}, y^{k}\right) \notin \operatorname{int}\left(L\left(x^{k}, \gamma^{k}\right)\right) \Longrightarrow D\left(x^{k}, y^{k}\right)=\operatorname{dist}\left(\left(x^{k}, y^{k}\right), H^{k}\right)$, onde $H^{k}=\left\{(x, y): y=\varphi\left(x^{k}\right)+<\gamma^{k}, x-x^{k}>\right\}$

Seja $\left(v^{k}, w^{k}\right)$ o ponto de $H^{k}$ tal que:

$$
D\left(x^{k}, y^{k}\right)=\operatorname{dist}\left(\left(x^{k}, y^{k}\right), L\left(x^{k}, \gamma^{k}\right)\right)=\operatorname{dist}\left(\left(x^{k}, y^{k}\right),\left(v^{k}, w^{k}\right)\right)
$$


Como $\left(v^{k}, w^{k}\right) \in H^{k}$, temos que $w^{k}=\varphi\left(x^{k}\right)+<\gamma^{k}, v^{k}-x^{k}>$.

Mas $D\left(x^{k}, y^{k}\right)=\operatorname{dist}\left(\left(x^{k}, y^{k}\right),\left(v^{k}, w^{k}\right)\right)=\sqrt{\left\|x^{k}-v^{k}\right\|^{2}+\left\|y^{k}-w^{k}\right\|^{2}}$, e como $D\left(x^{k}, y^{k}\right) \rightarrow 0$, vem que:

$$
\begin{aligned}
& \left\|x^{k}-v^{k}\right\| \rightarrow 0 \Longrightarrow v^{k} \rightarrow \bar{x} \\
& \left\|y^{k}-w^{k}\right\| \rightarrow 0 \Longrightarrow w^{k} \rightarrow \bar{y}
\end{aligned}
$$

Por outro lado, pelo teorema 2.4, $\exists \bar{k}$ tal que $\forall k>\bar{k} \quad\left\|\gamma^{k}\right\| \leq \bar{M}$

$$
\begin{array}{lll}
w^{k} & =\varphi\left(x^{k}\right) & +<\gamma^{k}, v^{k}-x^{k}> \\
\downarrow(2) & \downarrow \downarrow \text { continuidade de } \varphi & \downarrow(3) \mathrm{e}(1) \\
\bar{y} & =\varphi(\bar{x}) & +
\end{array}
$$

Teorema 2.7 Seja $\delta: X \backslash X^{*} \rightarrow \mathbb{R}$, dada por:

$\delta(x, y)=\max \{D(x, y), E(x, y)\}$

onde

$D(x, y)=\operatorname{dist}\left((x, y), L\left(x, \gamma_{x}\right)\right)$ para $\gamma_{x}$ subgradiente de $\varphi$ em $x$

$E(x, y)=(y-\varphi(x))+\frac{(p-1) \varphi(x)-\varphi^{*}}{p}$ para $\varphi^{*}$ valor ótimo de $\left(P^{\prime}\right)$ e $p>1$.

Então $\delta$ é um separador de $X^{*}$.

Demonstração

a) Primeiro provemos que $\delta(x, y) \geq 0, \quad \forall(x, y) \in X \backslash X^{*}$.

Seja $(x, y) \in X \backslash X^{*}$. Logo $\delta(x, y) \geq D(x, y) \geq 0$

b) Agora provemos que se $\lim _{k \rightarrow \infty}\left(x^{k}, y^{k}\right)=(\bar{x}, \bar{y})$ e $\lim _{k \rightarrow \infty} \delta\left(x^{k}, y^{k}\right)=$ 0 , então $(\bar{x}, \bar{y}) \in X^{*}$.

Consideremos as seguintes subsequências:

$K^{+}=\left\{k \in \mathbb{N}: y^{k}>\varphi\left(x^{k}\right)\right\}$ e $K^{-}=\left\{k \in \mathbb{N}: y^{k} \leq \varphi\left(x^{k}\right)\right\}$

10 caso: $\left|K^{+}\right|=\infty$ 
Neste caso, $\left\{\left(x^{k}, y^{k}\right)\right\}_{k \in K^{+}} \rightarrow(\bar{x}, \bar{y})$ e $\delta\left(x^{k}, y^{k}\right) \rightarrow 0$, com $y^{k}>\varphi\left(x^{k}\right)$.

Consideremos neste caso, apenas $k \in K^{+}$.

Como $y^{k}>\varphi\left(x^{k}\right)$

$$
\left(x^{k}, y^{k}\right) \in L\left(x^{k}, \gamma^{k}\right) \Longrightarrow D\left(x^{k}, y^{k}\right)=\operatorname{dist}\left(\left(x^{k}, y^{k}\right), L\left(x^{k}, \gamma^{k}\right)\right)=0
$$

Além disso, como $y^{k}>\varphi\left(x^{k}\right), \varphi\left(x^{k}\right) \geq \varphi^{*}$ e $p>1$,

$$
E\left(x^{k}, y^{k}\right)=\left(y^{k}-\varphi\left(x^{k}\right)\right)+\frac{(p-1)\left(\varphi\left(x^{k}\right)-\varphi^{*}\right)}{p}>0
$$

De (i) e (ii) vem que $\delta\left(x^{k}, y^{k}\right)=E\left(x^{k}, y^{k}\right)$.

Por continuidade de $\varphi$, e como $y^{k}-\varphi\left(x^{k}\right)>0$, temos que $\bar{y}-\varphi(\bar{x}) \geq 0 \quad$ (iii). Como $\delta\left(x^{k}, y^{k}\right) \rightarrow 0$, temos que $E\left(x^{k}, y^{k}\right) \rightarrow 0$, ou seja:

$$
\begin{array}{cll}
E\left(x^{k}, y^{k}\right) & =\left(y^{k}-\varphi\left(x^{k}\right)\right) & +\frac{(p-1)\left(\varphi\left(x^{k}\right)-\varphi^{*}\right)}{p} \\
\downarrow & & \downarrow \text { continuidade de } \varphi \\
0 & =(\bar{y}-\varphi(\bar{x})) & +\frac{(p-1)\left(\varphi(\bar{x})-\varphi^{*}\right)}{p}(i v)
\end{array}
$$

Como $(\bar{y}-\varphi(\bar{x})) \geq 0$ e $\frac{(p-1)\left(\varphi(\bar{x})-\varphi^{*}\right)}{p} \geq 0$, de $(i v)$ vem que $\bar{y}-\varphi(\bar{x})=0$ e $\frac{(p-1)\left(\varphi(\bar{x})-\varphi^{*}\right)}{p}=0$.

Logo, $\bar{y}=\varphi(\bar{x})=\varphi^{*} \Longrightarrow(\bar{x}, \bar{y}) \in X^{*}$.

$2^{\text {o }}$ caso: $\left|K^{-}\right|=\infty$

Neste caso, $\left\{\left(x^{k}, y^{k}\right)\right\}_{k \in K^{-}} \rightarrow(\bar{x}, \bar{y})$ e $\delta\left(x^{k}, y^{k}\right) \rightarrow 0, \operatorname{com} y^{k} \leq \varphi\left(x^{k}\right)$.

Consideremos neste caso, apenas $k \in K^{-}$.

Pela definição de $\delta(\cdot), \quad \delta\left(x^{k}, y^{k}\right) \geq D\left(x^{k}, y^{k}\right) \geq 0$, e como $\delta\left(x^{k}, y^{k}\right) \rightarrow 0$, então $D\left(x^{k}, y^{k}\right) \rightarrow 0$.

Portanto, pelo lema $2.2, \bar{y}=\varphi(\bar{x})$

Por outro lado temos que 


$$
\begin{aligned}
& E\left(x^{k}, y^{k}\right) \quad \rightarrow \quad E(\bar{x}, \bar{y})=(\bar{y}-\varphi(\bar{x}))+\frac{(p-1)\left(\varphi(\bar{x})-\varphi^{*}\right)}{p} \\
& \text { continuidade de } \varphi=\frac{(p-1)\left(\varphi(\bar{x})-\varphi^{*}\right)}{p} \geq 0
\end{aligned}
$$

Pela definição de $\delta(\cdot), \quad \delta\left(x^{k}, y^{k}\right) \geq E\left(x^{k}, y^{k}\right) \geq 0$, e como $\delta\left(x^{k}, y^{k}\right) \rightarrow 0$, então $E\left(x^{k}, y^{k}\right) \rightarrow 0$.

Logo, $E(\bar{x}, \bar{y})=\frac{(p-1)\left(\varphi(\bar{x})-\varphi^{*}\right)}{p}=0 \Longrightarrow \varphi(\bar{x})=\varphi^{*} \quad(i i)$.

De $\left(\right.$ i) e (ii) temos que $\bar{y}=\varphi(\bar{x})=\varphi^{*} \Longrightarrow(\bar{x}, \bar{y}) \in X^{*}$.

Teorema 2.8 Sejam $\left(x^{k}, y^{k}\right)$ e $C^{k}$ gerados pelo algoritmo, e $\delta$ o separador definido no teorema anterior. Então:

$$
C^{k} \cap B\left(\left(x^{k}, y^{k}\right), \delta\left(x^{k}, y^{k}\right)\right)=\emptyset
$$

Demonstração Como $C^{k}=C_{u}^{k} \cap C_{L}^{k} \cap X$, basta provarmos que uma das condições abaixo ocorre:

$$
\begin{gathered}
C_{u}^{k} \cap B\left(\left(x^{k}, y^{k}\right), \delta\left(x^{k}, y^{k}\right)\right)=\emptyset \text { ou } \\
C_{L}^{k} \cap B\left(\left(x^{k}, y^{k}\right), \delta\left(x^{k}, y^{k}\right)\right)=\emptyset
\end{gathered}
$$

10 caso : $\delta\left(x^{k}, y^{k}\right)=E\left(x^{k}, y^{k}\right)$

Temos que $C_{u}^{k}=\left\{(x, y): y \leq \varphi^{*}+\frac{u^{k+1}-\varphi^{*}}{p}\right\}$

Provemos que $\forall(x, y) \in B\left(\left(x^{k}, y^{k}\right), \delta\left(x^{k}, y^{k}\right)\right)$ é tal que $(x, y) \notin C_{u}^{k}$.

Seja $(x, y) \in B\left(\left(x^{k}, y^{k}\right), \delta\left(x^{k}, y^{k}\right)\right)$

Temos que $\left|y-y^{k}\right|<\left\|(x, y)-\left(x^{k}, y^{k}\right)\right\|<\delta\left(x^{k}, y^{k}\right)$. Dai temos

$$
\begin{aligned}
y>y^{k}-\delta\left(x^{k}, y^{k}\right) & =y^{k}-E\left(x^{k}, y^{k}\right) \\
& =y^{k}-\left(y^{k}-\varphi\left(x^{k}\right)\right)+\frac{(p-1)\left(\varphi\left(x^{k}\right)-\varphi^{*}\right)}{p} \\
& =\varphi\left(x^{k}\right)-\frac{(p-1)\left(\varphi\left(x^{k}\right)-\varphi^{*}\right)}{p} \\
& =\varphi^{*}+\frac{\left(\varphi\left(x^{k}\right)-\varphi^{*}\right)}{p}(i)
\end{aligned}
$$

Mas pela definição de $u^{k+1}=\min \left\{u^{k}, \varphi\left(x^{k}\right)\right\}$ temos $u^{k+1} \leq \varphi\left(x^{k}\right)$

Substituindo (ii) em (i), temos: 


$$
y>\varphi^{*}+\frac{\left(\varphi\left(x^{k}\right)-\varphi^{*}\right)}{p} \geq \varphi^{*}+\frac{\left(u^{k+1}-\varphi^{*}\right)}{p} \Longrightarrow(x, y) \notin C_{u}^{k}
$$

20 caso : $\delta\left(x^{k}, y^{k}\right)=D\left(x^{k}, y^{k}\right)=\operatorname{dist}\left(\left(x^{k}, y^{k}\right), L\left(x^{k}, \gamma^{k}\right)\right)$

Temos que $C_{L}^{k}=L\left(x^{k}, \gamma^{k}\right)$. Como $\left(x^{k}, y^{k}\right) \notin X^{*}$, e $\delta$ é separador, temos que $\delta\left(x^{k}, y^{k}\right)>0 \Longrightarrow D\left(x^{k}, y^{k}\right)>0$. Portanto $\left(x^{k}, y^{k}\right) \notin L\left(x^{k}, y^{k}\right)$.

Daí $B\left(\left(x^{k}, y^{k}\right), \operatorname{dist}\left(\left(x^{k}, y^{k}\right), L\left(x^{k}, y^{k}\right)\right)\right) \cap L\left(x^{k}, \gamma^{k}\right)=\emptyset$.

Logo:

$$
B\left(\left(x^{k}, y^{k}\right), \delta\left(x^{k}, y^{k}\right)\right) \cap C_{L}^{k}=\emptyset
$$

\subsubsection{Definição de um critério de parada prático}

O algoritmo pára quando um ponto com valor da função objetivo próximo ao valor ótimo for encontrado. É importante notar que não há garantia que o ponto encontrado estará próximo do conjunto de soluçóes ótimas, e sim que seu valor é próximo do ótimo. A figura abaixo mostra um exemplo em que esta afirmação fica bem clara.

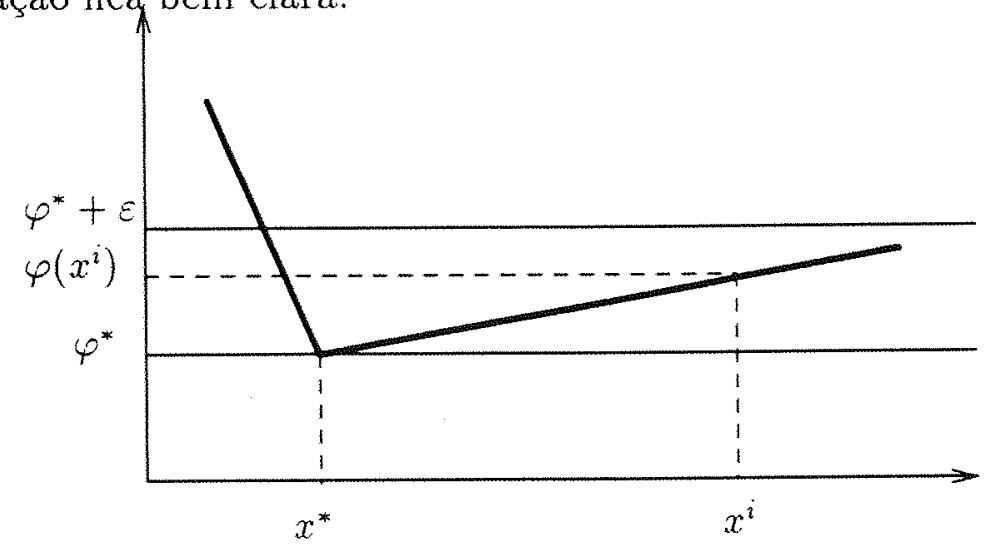

O teorema abaixo mostra uma sequência de limitantes inferiores do valor ótimo $\varphi^{*}$, gerada implicitamente pelo algoritmo. 
Teorema 2.9 Sejam $K$ o conjunto de indices de uma subsequência convergente do algoritmo e $p>1$ como definido no mesmo. Então a subsequência $\left\{l_{-}^{k}\right\}_{k \in K}$ definida por:

$$
l_{-}^{k}=\max \left\{\frac{p y^{k}-u^{k}}{(p-1)}, l_{-}^{k-1}\right\} \quad k \in K
$$

é tal que

$$
l_{-}^{k} \leq l^{k} \leq u^{k}
$$

onde $l^{k}=\min \left\{y:(x, y) \in D^{k}\right\}$. Além disso,

$$
\left\{u^{k}-l_{-}^{k}\right\}_{k \in K} \rightarrow 0
$$

Demonstração

Pela definição de $l_{-}^{k}, \exists i \leq k$ tal que

$$
l_{-}^{k}=\frac{p y^{i}-u^{i}}{(p-1)}
$$

Como $y^{i} \leq l^{i}+\frac{u^{i}-l^{i}}{p}$, onde $l^{i}=\min \left\{y:(x, y) \in D^{i}\right\}$, temos que

$$
l^{i} \geq \frac{p y^{i}-u^{i}}{(p-1)}=l_{-}^{k}
$$

Como $i \leq k$ então $D^{k} \subset D^{i}$ e pela definição de $l^{k}$, segue que $l^{k} \geq l^{i}$. Além disso, pelo definição de $D^{k}, u^{k} \geq l^{k}$. Assim temos que

$$
u^{k} \geq l^{k} \geq l^{i} \geq l_{-}^{k}
$$

Por outro lado, pela definição de $u^{k}, u^{k} \leq \varphi\left(x^{i}\right) \forall i<k$, então para todo $i<k$ temos

$$
0 \leq u^{k}-l_{-}^{k} \leq u^{k}-\frac{p y^{k}-u^{k}}{(p-1)} \leq \varphi\left(x^{i}\right)-\frac{p y^{k}-\varphi\left(x^{i}\right)}{(p-1)}=\frac{p\left(\varphi\left(x^{i}\right)-y^{k}\right)}{(p-1)}
$$


Daí

$$
0 \leq u^{k}-l_{-}^{k} \leq \frac{p\left(\varphi\left(x^{i}\right)-y^{k}\right)}{(p-1)} \quad \forall i<k
$$

Mas pela convergência do algoritmo $\left\{\left(x^{k}, y^{k}\right)\right\}_{k \in K} \rightarrow\left(x^{*}, \varphi\left(x^{*}\right)\right)$, portanto tomando $k=k_{j}$ e $i=k_{j-1}$

$$
\left\{\frac{p\left(\varphi\left(x^{k_{j-1}}\right)-y^{k_{j}}\right)}{(p-1)}\right\}_{k_{j} \in K} \rightarrow \frac{p\left(\varphi\left(x^{*}\right)-\varphi\left(x^{*}\right)\right)}{(p-1)}=0
$$

Logo $\left\{u^{k_{j}}-l_{-}^{k_{j}}\right\}_{k_{j} \in K^{r}} \rightarrow 0$

Corolário 2.3 Sejam $\left\{u^{k}\right\}_{k \in K}$ e $\left\{l_{-}^{k}\right\}_{k \in K}$ as sequências consideradas no teorema e $\varphi^{*}$ o valor o'timo de $(P C)$. Então

$$
\begin{aligned}
& \left\{u^{k}\right\}_{k \in K} \rightarrow \varphi^{*} \\
& \left\{l_{-}^{k}\right\}_{k \in K} \rightarrow \varphi^{*}
\end{aligned}
$$

Demonstração Pela definição de $u^{k}$, temos que $u^{k} \geq \varphi^{*}$ e $u^{k} \leq \varphi\left(x^{i}\right)$, $i<k, i, k \in K$. Como $\left\{\varphi\left(x^{i}\right)\right\}_{i \in K} \rightarrow \varphi^{*}$, vem que $\left\{u^{k}\right\}_{k \in K} \rightarrow \varphi^{*}$

Pelo teorema 2.9, temos que $\left\{u^{k}-l_{-}^{k}\right\}_{k \in K} \rightarrow 0$, e por $(i), \quad\left\{l_{-}^{k}\right\}_{k \in K} \rightarrow \varphi^{*}$

Pelos resultados apresentados, se desejamos encontrar um ponto com valor $\varphi(\cdot)$ a uma precisão de $\varepsilon$ de $\varphi^{*}$, podemos parar o algoritmo com $\left|u^{k}-l_{-}^{k}\right|<$ $\varepsilon$. Isto nos garante uma proximidade $\left|u^{k}-\varphi^{*}\right|<\varepsilon$ e pela definição de $u^{k}$, existe um ponto $\left(x^{i}, y^{i}\right)$ gerado pelo algoritmo tal que $\varphi\left(x^{i}\right)=u^{k} \operatorname{com} i<k$. 


\section{Capítulo 3}

\section{Eliminação de restrições}

No capítulo anterior estudamos o algoritmo de Goffin-Vial de um ponto de vista teórico, sem nos preocuparmos com eficiência prática. Neste capítulo trataremos de uma questão comum aos métodos de planos de corte: como eliminar restrições .

Em sua formulação original, o algoritmo em questão acrescenta a cada passo uma restrição na definição do poliedro usado no cálculo do centro analítico. Isto faz com que o tamanho do subproblema cresça demais tornando o método pouco eficiente.

Faremos então um estudo de como eliminar restrições sem perder garantia de convergência do algoritmo. Porém nao podemos garantir teoricamente, para uma função convexa qualquer, uma melhora na eficiência do algoritmo em termos de complexidade de tempo ou de iterações. Por isso trataremos na seção 3.2 de algumas heurísticas de eliminação que pretendem melhorar a eficiência do método.

No próximo capítulo, apresentaremos resultados computacionais para uma classe de funções específica, testando a utilização dessas heurísticas. 


\subsection{Garantia de convergência}

Para eliminarmos restrições garantindo convergência do algoritmo, utilizaremos fortemente a existência de subestimadores dos valores ótimos dos problemas linearizados. Consideremos então $l_{-}^{k}$, como visto anteriormente, definido por

$$
\begin{aligned}
& l_{-}^{1}=\frac{p y^{1}-u^{1}}{(p-1)} \\
& l_{-}^{k}=\max \left\{\frac{p y^{k}-u^{k}}{(p-1)}, l_{-}^{k-1}\right\} \quad k>1
\end{aligned}
$$

Como visto no capítulo anterior, temos que $l_{-}^{k} \leq l^{k}$ para $l^{k}$ valor ótimo do problema linearizado da iteração $k$.

Portanto, se acrescentarmos uma restrição adicional com este limitante inferior $L B\left(l_{-}^{k}\right)=\left\{(x, y) \in S \times \mathbb{R}: y \geq l_{-}^{k}\right\}$ não afetaremos a corretude e convergência do algoritmo original já que estaremos acrescentando uma restrição inativa.

Alteraremos então as restrições geradas pelo novo algoritmo como a seguir:

Algoritmo de Goffin-Vial com restrição de limitante inferior

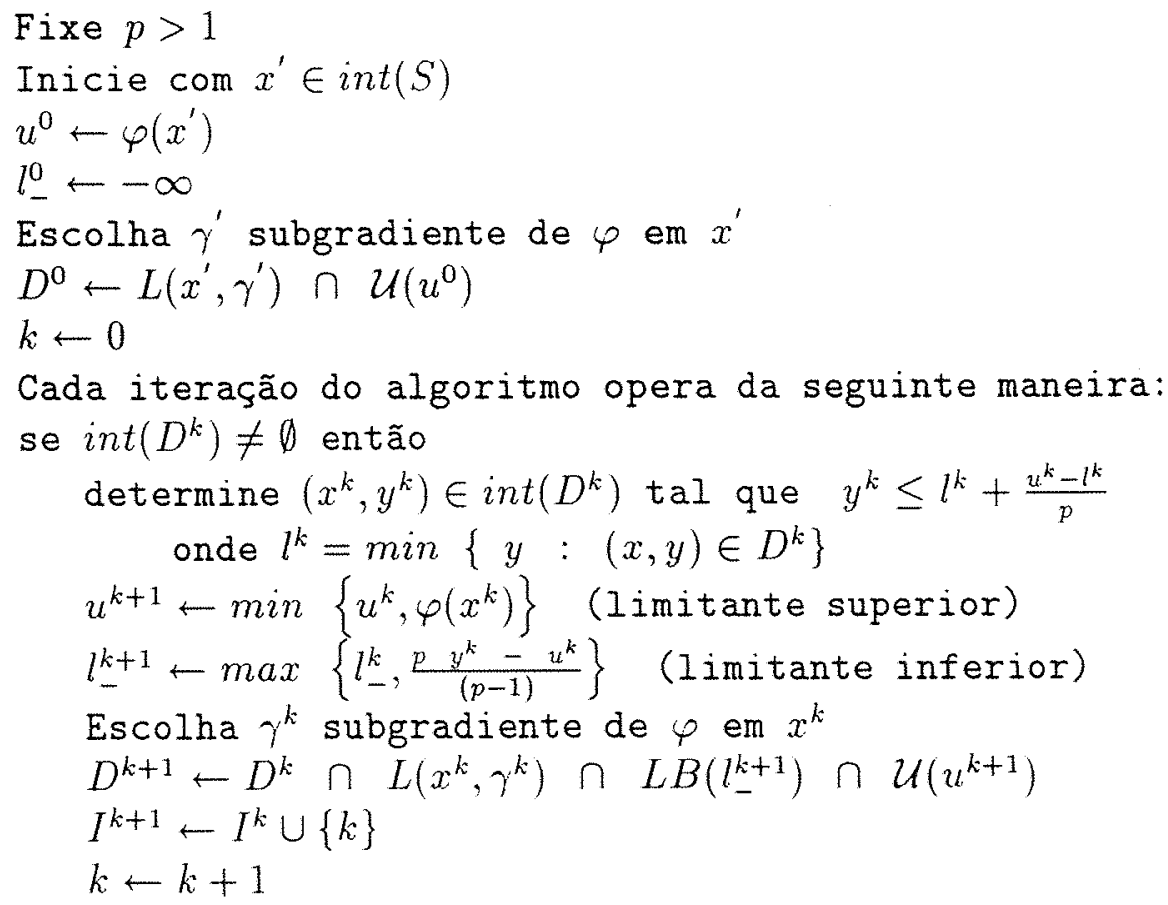


Uma observação simples, porém importante, é que é necessário manter apenas uma restrição de limitante superior e inferior, pois pela própria escolha desses, somente a última restrição acrescentada poderá estar ativa.

Portanto, quando falarmos em acréscimo de restrição de limitante superior e inferior, podemos entender como uma atualização dos valores que definem estas restriçôes. As restrições que serão eliminadas são aquelas provenientes de linearizações $\left(L\left(x^{k}, \gamma^{k}\right)\right)$.

\section{Método A de eliminação de restrições}

Em uma iteração $k$, este método elimina toda restrição $i$, associada a $\left(x^{i}, y^{i}\right)$, tal que $y^{i}$ está fora do intervalo $\left[l_{-}^{k}, u^{k}\right]$ e com uma distância de pelo menos o valor do separador $\left(\delta\left(x^{i}, y^{i}\right)\right)$.

Seja $k$ uma iteração, com o conjunto $D^{k}$ definido por:

$$
D^{k+1}=D^{k} \cap L B\left(l_{-}^{k+1}\right) \cap \mathcal{U}\left(u^{k+1}\right) \cap\left(\cap_{i \in I^{k}} L\left(x^{i}, \gamma^{i}\right)\right)
$$

onde $I^{k} \subset\{1,2, \cdots, k\}$ é o conjunto das restrições que nào foram eliminadas e $\delta$ é o separador utilizado para convergência do algoritmo.

Eliminaremos cada restrição $L\left(x^{i}, \gamma^{i}\right)$ associada a $\left(x^{i}, y^{i}\right)$ como mostrado a seguir:

$$
\begin{aligned}
E^{k} & \leftarrow \emptyset \\
\text { Para cada } i \in I^{k} & \\
& \text { se }\left(y^{i}-\delta\left(x^{i}, y^{i}\right) \geq u^{k}\right) \text { ou }\left(y^{i}+\delta\left(x^{i}, y^{i}\right) \leq l_{-}^{k}\right) \\
& \text { então } E^{k} \leftarrow E^{k} \cup\{i\} \\
I^{k+1} & \leftarrow I^{k} \backslash E^{k}
\end{aligned}
$$

Proposição 3.1 A convergência do algoritmo é mantida se utilizarmos o método $\mathbf{A}$ de eliminação de restriçôes. 


\section{Demonstração}

Seja $\left(x^{j}, y^{j}\right)$ gerado numa iteração $j$ pelo algoritmo. Provaremos que para todo $k>j$

$$
\left(x^{k}, y^{k}\right) \notin B\left(\left(x^{j}, y^{j}\right), \delta\left(x^{j}, y^{j}\right)\right)
$$

onde $\delta$ é o separador utilizado na demonstração de convergência do algoritmo.

Se $j \in I^{k}$, a restrição $L\left(x^{i}, \gamma^{i}\right)$ não foi eliminada, portanto segue que $\left(x^{k}, y^{k}\right) \notin B\left(\left(x^{j}, y^{j}\right), \delta\left(x^{j}, y^{j}\right)\right)$.

Se $j \notin I^{k}$, temos que para algum $i \leq k$

$$
\begin{aligned}
& \text { ou }(i) y^{j}-\delta\left(x^{j}\right) \geq u^{i} \\
& \text { ou }(i i) y^{j}+\delta\left(x^{j}\right) \leq l_{-}^{i}
\end{aligned}
$$

Como $u^{k} \leq u^{i}$ e $l_{-}^{k} \geq l_{-}^{i}$, para $k \geq i$, temos que

Se $(i)$ vale então $y^{j}-\delta\left(x^{j}\right) \geq u^{i} \geq u^{k}$. Logo

$$
B\left(\left(x^{j}, y^{j}\right), \delta\left(x^{j}, y^{j}\right)\right) \cap \mathcal{U}\left(u^{k}\right)=\emptyset
$$

Se (ii) vale, analogamente temos $y^{j}+\delta\left(x^{j}\right) \leq l_{-}^{i} \leq l_{-}^{k}$. Logo

$$
B\left(\left(x^{j}, y^{j}\right), \delta\left(x^{j}, y^{j}\right)\right) \cap L B\left(l_{-}^{k}\right)=\emptyset
$$

De $(i)$ ou (ii) vem entào que

$$
\left(x^{k}, y^{k}\right) \notin B\left(\left(x^{j}, y^{j}\right), \delta\left(x^{j}, y^{j}\right)\right) \quad \forall k>j
$$

Esta era a condição que garantia a convergência do algoritmo sem eliminaçôes. 


\section{Método $\mathbf{B}$ de eliminação de restrições}

Este método elimina todas as restrições sempre que o intervalo $u^{k}-l_{-}^{k}$ tenha caído de $\Delta$ em relação ao intervalo $u^{i}-l_{-}^{i}, i<k$, no qual foi feita a última eliminação deste tipo.

Fixemos $\triangle<1, G A P^{0} \leftarrow \infty$.

Seja $k$ uma iteração, com o conjunto $D^{k}$ definido por:

$$
D^{k+1}=D^{k} \cap L B\left(l_{-}^{k+1}\right) \cap \mathcal{U}\left(u^{k+1}\right) \cap\left(\cap_{i \in I^{k}} L\left(x^{i}, \gamma^{i}\right)\right)
$$

onde $I^{k} \subset\{1,2, \cdots, k\}$ é o conjunto das restrições que não foram eliminadas e $\delta$ é o separador utilizado para convergência do algoritmo.

Eliminaremos as restriçôes como mostrado a seguir:

$$
\begin{aligned}
\text { se } u^{k}-l_{-}^{k}<\Delta G A P^{k} \\
\text { então } \\
I^{k+1} \leftarrow \emptyset \\
G A P^{k+1} \leftarrow u^{k}-l_{-}^{k} \\
\text { senão } \\
I^{k+1} \leftarrow I^{k} \\
G A P^{k+1} \leftarrow G A P^{k}
\end{aligned}
$$

Proposição 3.2 A convergência do algoritmo é mantida se utilizarmos o método $\mathrm{B}$ de eliminação de restrições.

Demonstração

Seja $K=\left\{k \in \mathbb{N}: I^{k+1}=\emptyset\right\}$.

10 caso: $|K|=n<\infty$

Neste caso, não serào feitas mais eliminaçòes para $i>k_{n}$. Podemos considerar a sequência gerada a partir de $k_{n}$ como uma sequência sem eliminações, e a convergência segue do algoritmo sem eliminações. 
20 caso: $|K|=\infty$

Neste caso, temos uma sequência: $\left\{G A P^{k_{i}}\right\}_{k_{i} \in K}$ tal que

$$
0<G A P^{k_{i}}<\Delta G A P^{k_{i-1}}
$$

Como $\Delta<1,\left\{G A P^{k_{i}}\right\}_{k_{i} \in K} \rightarrow 0$ ou seja,

$$
\left\{u^{k_{i}}-l_{-}^{k_{i}}\right\}_{k_{i} \in K} \rightarrow 0
$$

Pela definição do algoritmo, se $\varphi^{*}$ é o valor ótimo, $\left\{u^{k}-l_{-}^{k}\right\}_{k \in K} \rightarrow 0$. Então $l_{-}^{k} \rightarrow \varphi^{*}$ e $u^{k} \rightarrow \varphi^{*}$. Mas como $u^{k} \geq \varphi^{*} \geq l_{-}^{k} \forall k \in \mathbb{N}$, segue que $\left\{u^{k_{i}}\right\}_{k_{i} \in K} \rightarrow \varphi^{*}$. E como $u^{k_{i}}=\varphi\left(x^{j}\right)$ com $j<k_{i}$, da continuidade de $\varphi(\cdot)$ e pela compacidade de $S$ segue a tese.

Pela demonstração da convergência do método $\mathbf{B}$, podemos notar que se combinamos os métodos de $\mathbf{A}$ e $\mathbf{B}$, como mostrado a seguir, a convergência é mantida.

Método AB de eliminação de restrições

Este método combina os métodos $\mathbf{A}$ e $\mathbf{B}$.

Fixemos $\triangle<1, G A P^{0} \leftarrow \infty$.

Seja $k$ uma iteração, com o conjunto $D^{k}$ definido por:

$$
D^{k+1}=D^{k} \cap L B\left(l_{-}^{k+1}\right) \cap \mathcal{U}\left(u^{k+1}\right) \cap\left(\cap_{i \in I^{k}} L\left(x^{i}, \gamma^{i}\right)\right)
$$

onde $I^{k} \subset\{1,2, \cdots, k\}$ é o conjunto das restrições que não foram eliminadas e $\delta$ é o separador utilizado para convergência do algoritmo.

Eliminaremos cada restrição $L\left(x^{i}, \gamma^{i}\right)$ associada a $\left(x^{i}, y^{i}\right)$ como mostrado a seguir: 


$$
\begin{aligned}
& \text { se } u^{k}-l_{-}^{k}<\Delta G A P^{k} \\
& \text { então } \\
& I^{k+1} \leftarrow \emptyset \\
& G A P^{k+1} \leftarrow u^{k}-l_{-}^{k} \\
& \text { senão } \\
& E^{k} \leftarrow \emptyset \\
& \text { Para cada } i \in I^{k} \\
& \text { se }\left(y^{i}-\delta\left(x^{i}, y^{i}\right) \geq u^{k}\right) \text { ou }\left(y^{i}+\delta\left(x^{i}, y^{i}\right) \leq l_{-}^{k}\right) \\
& \text { então } E^{k} \leftarrow E^{k} \cup\{i\} \\
& I^{k+1} \leftarrow I^{k} \backslash E^{k}
\end{aligned}
$$

Proposição 3.3 O método AB mantém a convergência do algoritmo.

\section{Demonstração}

A demonstração da proposição segue analogamente à do método B. Para o $1^{\mathrm{O}}$ caso, a convergência sai da convergência do método $\mathbf{A}$, e o $2^{\mathrm{O}}$ caso fica inalterado.

As estratégias de eliminação de restriçôes descritas anteriormente podem ser expandidas para variações intermediárias que não eliminam necessariamente todas as restrições que satisfazem as condições da eliminação. É claro que se o algoritmo converge sem uma restrição ele certamente convergirá com ela .

A escolha de quais restriçôes dentre as elimináveis serão realmente cortadas, é uma questão de determinar heurísticas que funcionem bem na prática. Este é o assunto da próxima seção.

\subsection{Controle de eliminação de restrições}

O principal problema em fazermos eliminações excessivas é que eventualmente o número de iterações pode aumentar demais piorando o tempo total do 
algoritmo, apesar do tempo por iteração ter reduzido.

Boas estratégias de eliminação dependem de heurísticas que permitam eliminar um número suficiente de restrições de modo a haver uma economia de tempo por iteração e ao mesmo tempo garantam um crescimento controlado do número de iterações. Escolher heurísticas que funcionem bem na prática é mais fácil quando temos um conhecimento maior da estrutura do problema a ser resolvido.

Analisaremos alguns fatores para determinar estratégias de eliminação.

- Quais, dentre as restrições, melhor aproximam o poliedro definido por todas

A definição do que é uma aproximação melhor do poliedro não é muito precisa, porém a idéia seria determinar quais restriçôes mais contribuem para a definiçâo do poliedro. Este é um problema difícil de ser tratado formalmente e além disso, até problemas mais simples, como eliminação de restrições inativas, podem requerer um esforço computacional que não justificaria seu uso. Lembremos que o poliedro em questão tem uma característica especial: ser obtido através de aproximações lineares da função $\varphi$.

Exemplos de critérios que podem ser utilizados são: 1) manter as últimas restrições geradas; 2) manter as restrições que são aproximações lineares de pontos de menor valor de função objetivo

É claro que é possível construir exemplos nos quais os critérios apresentados não dão bons resultados.

\section{- Quantas restrições devemos manter}

A partir de um critério que defina a prioridade das restriçôes, precisamos escolher quantas restrições serão mantidas. Gostaríamos de encontrar um número ótimo de restrições levando em conta que restrições demais aumentamo tempo de uma iteração e restrições a menos podem aumentar muito o número de iteraçôes. Não sabemos como escolher o número adequado, porém os casos extremos (manter todas as restrições ou não manter nenhuma), parecem inadequados.

Uma possibilidade seria mantermos um número de restriçôes polinomial na dimensâo do espaço. No caso de nossas implementações, escolhemos duas possibilidades: manter $n+1$ e $2 n$ restrições. A motivação 
da primeira escolha foi o fato que o número mínimo de restrições que podem definir um conjunto limitado no $\mathbb{R}^{n}$ é $n+1$, e nos parece desejável que o conjunto definido pelas restrições de linearização, mais a restrição de limitante superior, seja limitado.

A segunda escolha foi baseada em testes computacionais com alguns exemplos de funções quadráticas no $\mathbb{R}^{2}$. Nestes exemplos o conjunto de restrições que definiam os poliedros a cada passo formavam aproximadamente um "box" no $\mathbb{R}^{n}$, que é definido por $2 n$ restrições. Este fenômeno está relacionado com a forte simetria nos exemplos escolhidos, porém aproveitamos para testar esta idéia com uma massa de testes mais completa.

\section{- Passo da eliminação}

Muitas vezes eliminar restrições a cada iteração pode resultar em dois problemas. O primeiro é que se o tempo envolvido em cálculos para fazer eliminações for grande, pode ocorrer que o processamento desses cálculos a cada iteração aumente muito o tempo total do algoritmo. Outro problema é que, dependendo do critério de seleção de quais restrições serão mantidas, esperar mais para eliminar possa ser bom no sentido de poder armazenar mais "informação" a respeito da função, antes de selecionar as restriçôes melhores.

- Determinação do separador

A escolha do separador utilizado, no caso de método de eliminação tipo $\mathbf{A}$ ou $\mathrm{AB}$, pode afetar diretamente as eliminações. Usar qualquer separador $\delta^{\prime} \leq \delta$, onde $\delta$ é o separador usado na demonstração de convergência do algoritmo, garantirá a convergência do mesmo. Lembrando que múltiplo escalar de separador é separador, podemos utilizar qualquer separador $\bar{\delta}=\alpha \delta$, para $\alpha \leq 1$. Separadores menores permitirão mais eliminações do tipo $\mathbf{A}$.

As variações possiveis em estratégias são incontáveis. Uma estratégia pode ser muito boa para um certo tipo de funçôes e piorar a performance do algoritmo para outros.

No próximo capítulo exibiremos resultados computacionais para funções quadráticas convexas sob restriçôes de box, usando variaçôes das eliminaçôes descritas neste capítulo. 


\section{Capítulo 4}

\section{Resultados Computacionais}

Implementamos o algoritmo de Goffin-Vial em sua formulação original e com quatro estratégias de eliminação que serão descritas neste capítulo. Os programas foram escritos em linguagem de programação $C$ e executados em equipamentos tipo SUN Workstations.

O objetivo principal da implementação era testarmos o efeito de eliminação de restrições sobre o algoritmo de Goffin-Vial. Poderíamos escolher uma massa de testes com tipos de funções e poliedros bem variados, já que a única hipótese sobre a função é sua convexidade. Porém, optamos por fazer uma análise mais detalhada para um tipo específico de problema, de modo a compreender melhor a influência de certos parâmetros da definição do problema na "performance" do algoritmo.

Escolhemos como massa de teste funções quadráticas convexas sob restrições de "box", geradas aleatoriamente, fixando alguns parâmetros. A geração dos testes, proposta por Moré e Toraldo [14], será descrita mais adiante.

Além da análise de eliminaçòes sobre o método, pretendíamos também compará-lo com outros métodos para este tipo de problema. Os métodos para programação convexa de Moré e Toraldo [14] e de Friedlander e Martinez [4] utilizam uma mesma geração de exemplos, o que padronizaria os testes para comparação. Ambos os métodos combinam estratégias de conjunto ativo com métodos de gradiente projetado, sendo que o algoritmo de Friedlander e Martineż nảis necessita de hipóteses de não-degenerescência dual e de não- 
singularidade da matriz Hessiana para obter convergência finita.

Como os métodos citados utilizam ferramentas muito diferentes das nossas, não faria sentido compará-los com a nossa implementação com base nos resultados publicados em termos de número de iterações e faces visitadas. Optamos, então, por fazer uma comparação de tempo com o software gentilmente cedido por Friedlander e Martinez, implementado em mesmo tipo de equipamento. Observamos que essa implementação resolvia problemas de grande porte, com 1000 variáveis, em poucos segundos, sendo que a nossa gastava minutos para exemplos de 20 variáveis. Além disso, no nosso caso o aumento de tempo com o número de variáveis é bastante acentuado, como veremos na seção 4.3. Alguma discrepância de eficiência entre esses dois métodos era esperada, já que o de Friedlander e Martinez utiliza fortemente a estrutura de restriçôes de box e o fato da função ser quadrática. Portanto, isso não inviabiliza a utilização do algoritmo de Goffin-Vial para outros tipos de função, para as quais não existem métodos tão eficientes.

O nosso objetivo central, que era testar o quanto a eliminação de restrições pode melhorar a eficiência do algoritmo, teve bons resultados, que estão descritos na seção 4.4. Outro aspecto interessante é que pudemos também observar como a variação de certos parâmetros que descrevem as quadráticas afetaram a nossa implementação do algoritmo, dando-nos um pouco mais de informação sobre como ele funciona na prática.

\subsection{Aspectos de implementação}

\subsubsection{Inicialização da rotina de cálculo do centro}

$\mathrm{O}$ algoritmo utilizado para o cálculo do centro analítico requer um ponto interior ao poliedro $D$ inicial viável. Como a cada passo do algoritmo o ponto anterior já não é mais viável, precisamos de uma re-inicialização.

O método utilizado consiste em obter um ponto na epígrafe de $\varphi$ que seja viável e, após fazer um ajuste no limitante superior $(u)$, obter um ponto interior à epígrafe e ao poliedro $D$. Esta inicialização é bastante simples e barata em termos computacionais, porém o ponto encontrado não está próximo ao 
centro analítico.

Um outro método interessante para a re-inicialização foi desenvolvido por Delgado [2]. Esse método é geral para algoritmos de planos de corte que tenham como sub-problemas a determinação de centros analíticos. Apesar da inicialização ser mais complexa, ela fornece um ponto próximo ao centro analítico, o que irá certamente reduzir os passos no cálculo do centro. Tal método não foi implementado em nosso trabalho, porém pode ser visto como uma possível melhoria dos resultados aqui apresentados.

\subsubsection{Cálculo do centro analítico}

Utilizamos o método de Newton-Raphson descrito no capítulo 1. A cada passo, precisamos achar a direção de minimização da aproximação quadrática e fazer uma minimização unidirecional da função centro nessa direção.

Lembremos que a direção de minimização a partir de $x$ é dada por:

$$
h=-H^{-1} g
$$

onde $H=H(x, u)$ é a hessiana de $f_{u}(\cdot)$ em $x$

$$
g=g(x, u) \text { é o gradiente de } f_{u}(\cdot) \text { em } x
$$

Utilizamos o método de Gauss para resolver o sistema $H h=-g$, obtendo $h$.

Para fazer a minimização unidirecional, consideremos a função:

$$
\theta(\lambda)=f_{u}(x+\lambda h)
$$

Queremos encontrar $\lambda^{*}$ que minimize $\theta(\cdot)$ para $\lambda>0 \mathrm{e}$, como $\theta(\cdot)$ é estritamente convexa temos que

$$
\lambda^{*} \operatorname{minimiza} \theta(\cdot) \Leftrightarrow \theta^{\prime}\left(\lambda^{*}\right)=0
$$

Iniciamos a busca com um intervalo $\left(0, \lambda^{+}\right)$tal que $\theta^{\prime}(0)<0$ e $\theta^{\prime}\left(\lambda^{+}\right)>0$, e prosseguimos fazendo dicotomias até que o intervalo seja menor que um $\varepsilon$ dado, obtendo um ponto $\bar{\lambda}$ tal que $\left|\bar{\lambda}-\lambda^{*}\right|<\frac{\varepsilon}{2}$. No nosso caso, utilizamos 
$\varepsilon=10^{-4}$

Como critério de parada para o algoritmo de Newton-Raphson utilizamos $\delta(x, h)=\|h\|_{H}<0.1$.

\subsubsection{Critério de parada do algoritmo de Goffin-Vial}

Lembremos que o algoritmo pára quando $u^{k}-l_{-}^{k}$ está suficientemente pequeno. Utilizamos a seguinte combinação de critérios absoluto e relativo:

Se $\left|l_{-}^{k}\right|<=1.0$ então pare se $u^{k}-l_{-}^{k}<\varepsilon$

senão pare se $\frac{u^{k}-l_{-k}^{k}}{\left|\underline{I}_{-}^{k}\right|}<\varepsilon^{2}$

Utilizamos $\varepsilon^{-10^{-6}}$.

\subsection{Problemas Teste}

\subsubsection{Geração dos dados}

Testamos nossos algoritmos com funçôes quadráticas convexas, sujeitas a restrições de "box". Utilizamos a geração de dados descrita em Moré e Toraldo [14]. Esta geração é aleatória, fixando alguns parâmetros que podem influenciar a performance de algoritmos sobre este tipo de problemas:

- número de variáveis : $n$

- número de restrições ativas na solução ótima: na $\left(x^{*}\right)$

- número de restriçôes ativas no ponto inicial: $n a\left(x^{0}\right)$ (no nosso caso utilizamos restrições "quase ativas", já que $x^{0}$ deve ser interior ao box)

- número de condicionamento da matriz que define a quadrática: ncond

- quantidade de degenerescência de $x^{*}$, que está associada a quão perto de zero estão as derivadas parciais das componentes de $x^{*}$ que são ativas: ndeg 
Descrevemos a seguir como gerar problemas aleatórios, variando esses parâmetros. Consideremos o problema:

$$
(P Q) \quad\left\{\begin{array}{l}
\min q(x) \\
l \leq x \leq u
\end{array}\right.
$$

onde $q: \mathbb{R}^{n} \rightarrow \mathbb{R}$ é uma funçào quadrática convexa e $l, u \in \mathbb{R}^{n}$ especificam os limitantes do box.

A função $q$ pode ser escrita da seguinte forma:

$$
q(x)=\frac{1}{2} x^{T} A x-b^{T} x
$$

onde $A$ é definida por $A=Y D Y, \quad Y=I-\frac{2}{\|y\|^{2}} y y^{T}$ e $D$ é uma matriz diagonal positiva.

Note que $\nabla q(x)=A x-b$.

Observe também que $x^{*}$ tal que $l \leq x^{*} \leq u$ é solução ótima de $(P Q)$ se e somente se:

$$
\partial_{i} q\left(x^{*}\right)= \begin{cases}=0, & \text { se } l_{i}<x_{i}^{*}<u_{i} \\ \geq 0, & \text { se } l_{i}=x_{i}^{*}<u_{i} \\ \leq 0, & \text { se } l_{i}<x_{i}^{*}=u_{i}\end{cases}
$$

Para gerar um problema deste tipo, serào fornecidos os parâmetros: $n$, ncond, $n a\left(x^{0}\right), n a\left(x^{*}\right)$ e ndeg, e serão determinados:

1. os limitantes do box: $l$ e $u$

2. a matriz $A$

3. o vetor $b$

4. o ponto inicial $x^{0}$ 


\section{Geração de $l$ e $u$}

Geram-se aleatoriamente as componentes da solução ótima $x^{*}$.

Determina-se o conjunto de restriçooes ativas em $x^{*}, \mathcal{A}\left(x^{*}\right) \subset\{1,2, \cdots, n\}$, dependente de $n a\left(x^{*}\right)$, através da geração de números aleatórios $\delta_{i}$ em $(0,1) i=1, \cdots, n$ e da seleção de $i$ para $\mathcal{A}\left(x^{*}\right)$ se $\delta_{i} \leq \frac{n a\left(x^{*}\right)}{n}$.

Definimos:

para $i \notin \mathcal{A}\left(x^{*}\right) \quad l_{i}=-1$ e $u_{i}=+1$

para $i \in \mathcal{A}\left(x^{*}\right) \quad l_{i}=x_{i}^{*}$ e $u_{i}=+1$ ou $l_{i}=-1$ e $u_{i}=x_{i}^{*}$.

Aproximadamente metade dos índices $i$ em $\mathcal{A}\left(x^{*}\right)$ devem ter $l_{i}=x_{i}^{*}$ e a outra metade, $u_{i}=x_{i}^{*}$.

\section{Geração da matriz $A$}

A matriz $A$ depende do vetor $y$ e da matriz diagonal $D$, como visto anteriormente. As componentes de $y$ são geradas aleatoriamente em $(-1,1)$. A matriz diagonal $D$ tem a $i$-ésima componente, $d_{i}$, definida por:

$$
\log d_{i}=\left(\frac{i-1}{n-1}\right) n \text { cond }
$$

Isto gera uma matriz $A$ definida positiva com o $\log (\operatorname{cond}(A))=n$ cond. $\mathrm{O}$ parâmetro ncond especifica o número de condicionamento de $A$. Por exemplo, se $n$ cond $=0$ entào $A$ é a identidade.

\section{Geração do vetor $b$}

Como $\nabla q(x)=A x-b$, tendo $A$ e uma solução ótima $x^{*}$ gerada antei iormente e gerando $\nabla q\left(x^{*}\right)$, determinamos $b=A x^{*}-\nabla q\left(x^{*}\right)$. Logo resta gerar $\nabla q\left(x^{*}\right)$, sabendo que deve satisfazer as condições 4.2.1. O sinal de $\partial_{i} q\left(x^{*}\right)$ é dado por:

$$
\partial_{i} q\left(x^{*}\right)\left\{\begin{array}{l}
=0, \text { se } l_{i}<x_{i}^{*}<u_{i} \\
>0, \text { se } l_{i}=x_{i}^{*}<u_{i} \\
<0, \text { se } l_{i}<x_{i}^{*}=u_{i}
\end{array}\right.
$$


Precisamos ainda determinar $\left|\partial_{i} q\left(x^{*}\right)\right|$, que será calculado a partir de $\mu_{i}$ gerado aleatoriamente entre $(0,1)$ e $n$ deg que especifica a "quantidade de degenerescência", no sentido de que quanto maior ndeg, mais próximo de zero estarão os $\partial_{i} q\left(x^{*}\right)$ tais que $i \in \mathcal{A}\left(x^{*}\right)$. Então fazemos:

$$
\left|\partial_{i} q\left(x^{*}\right)\right|=10^{-\mu_{i} n d e g}, i \in \mathcal{A}\left(x^{*}\right)
$$

Especificado completamente $\nabla q\left(x^{*}\right)$, podemos agora calcular $b$.

\section{Ponto inicial $x^{0}$}

Seleciona-se o conjunto de restriçôes "quase" ativas $\mathcal{A}\left(x^{0}\right)$, gerando aleatoriamente $\varrho_{i} \in(0,1)$ e selecionando $i$ para $\mathcal{A}\left(x^{0}\right)$ se $\varrho_{i} \leq \frac{n a\left(x^{0}\right)}{n}$. Então determina-se $x^{0}$ da seguinte forma:

para $i \notin \mathcal{A}\left(x^{0}\right) \quad x_{i}^{0}=\left(l_{i}+u_{i}\right) / 2$

para $i \in \mathcal{A}\left(x^{0}\right) \quad x_{i}^{0}=l_{i}+0.1\left(u_{i}-l_{i}\right) \quad$ ou $\quad x_{i}^{0}=u_{i}-0.1\left(u_{i}-l_{i}\right)$

Aproximadamente metade dos índices $i$ em $\mathcal{A}\left(x^{0}\right)$ devem ter $x_{i}^{0}$ mais próximo de $l_{i}$ e a outra metade mais próximo de $u_{i}$.

\subsubsection{Variações utilizadas nos testes}

Testamos nossas implementações para a combinação das seguintes variações de parâmetros, sendo que geramos 2 testes para cada variaçào:

$$
\begin{array}{ll}
n: & 5,10,20 \\
n \text { cond: } & 0,3,6,12 \\
n \text { deg: } & 1,6,12 \\
n a\left(x^{*}\right): 0.1 n, 0.5 n, 0.9 n \\
n a\left(x^{0}\right): 0.1 n, 0.5 n, 0.9 n
\end{array}
$$

Isto nos dá, para cada n, 72 tipos de funçâo com 3 inicializaçôes diferentes para cada uma (variando $n a\left(x^{0}\right)$ ). Para $n=5$ e $n=10$ testamos então essas 216 variaçôes possiveis. Como o parâmetro na $\left(x^{0}\right)$ não influenciou os resultados, para $n=20$ utilizamos 72 testes, correspondendo a 72 funções diferentes sem variar o parâmetro na $\left(x^{0}\right)$. 


\subsection{Algoritmo sem eliminações: efeito dos parâmetros que definem as quadráticas}

O parâmetro que mais influenciou o tempo total do algoritmo foi a dimensão do espaço $(n)$. A tabela a seguir mostra o efeito de $n$ sobre o tempo total, número de iterações e número total de Hessianas calculadas no algoritmo. Cada entrada da tabela é a média aritmética dos valores de todos os testes para um certo $n$ (216 testes para $n=5$ e $n=10$, e 72 para $n=20$ ).

\begin{tabular}{|c|c|c|c|}
\hline $\mathrm{n}$ & $\begin{array}{c}\text { tempo } \\
\text { total }\end{array}$ & iteraçôes & Hessianas \\
\hline 5 & $2.59 \mathrm{~s}$ & 41 & 293 \\
10 & $24 \mathrm{~s}$ & 74 & 604 \\
20 & $272 \mathrm{~s}$ & 145 & 1314 \\
\hline
\end{tabular}

Lembremos que a cada iteração do algoritmo obtemos o centro analítico aproximado pelo método de Newton-Raphson, que calcula sucessivas direçôes baseadas no cálculo de Hessianas. Destacamos na tabela anterior o número de Hessianas calculadas, pois aí reside 50 a $80 \%$ do esforço computacional do algoritmo. Observemos também que o cálculo de Hessianas da função centro utiliza $\mathcal{O}\left(n^{2} r\right)$ operações aritméticas, onde $r$ é o número de restrições que definem o poliedro, que por sua vez aumenta a cada iteração. Por isso, o tempo envolvido no cálculo das Hessianas aumenta com o número de iterações não só por calcularmos mais Hessianas mas também pelo cálculo gastar mais tempo.

A tabela a seguir mostra a distribuição do tempo gasto pelo algoritmo em suas principais tarefas, para exemplos $\operatorname{com} n=5,10$ e 20 . 


\begin{tabular}{|c|c|c|c|}
\hline $\mathrm{n}$ & 5 & 10 & 20 \\
\hline $\begin{array}{c}\text { Cálculo das } \\
\text { Hessianas }\end{array}$ & $50 \%$ & $70 \%$ & $80 \%$ \\
\hline $\begin{array}{c}\text { Resolução do } \\
\text { Sistema Linear }\end{array}$ & $5 \%$ & $7 \%$ & $7 \%$ \\
\hline $\begin{array}{c}\text { Busca } \\
\text { Unidirecional }\end{array}$ & $32 \%$ & $15 \%$ & $6 \%$ \\
\hline Outros & $13 \%$ & $8 \%$ & $7 \%$ \\
\hline
\end{tabular}

Além da média aritmética utilizada nas tabelas utilizamos gráficos que mostram a distribuição dos dados para cada parâmetro variado. Os "boxes" que aparecem nos gráficos podem ser interpretados com explicaremos a seguir. Suponhamos que os dados para uma certa entrada no eixo das abcissas estão ordenados segundo o seu valor no eixo das ordenadas. A linha central do box representa a mediana de todos os valores. As linhas inferiores e superiores do box representam o quartil inferior e superior dos dados, que são, respectivamente, os valores minimo e máximo desprezando $25 \%$ dos menores e maiores valores. Deste modo, o box representa a distribuição de $50 \%$ dos dados. As linhas de prolongamento do box indicam os valores máximo e mínimo encontrados desde que estes não ultrapassem 1,5 vezes o intervalo interquartil. No caso de valores que ultrapassam este intervalo, eles serão indicados no gráfico como pontos isolados.

Já que os efeitos dos parâmetros se mostraram equivalentes para as variações de $n$, exibimos no decorrer do texto apenas os gráficos para 1 valor de $n$, e os demais podem ser encontrados no apêndice.

Os outros parâmetros que influenciaram bastante a performance do algoritmo foram o grau de condi-cionamento da matriz (medido por ncond) e o número de restrições ativas na solução ótima $\left(n a\left(x^{*}\right)\right)$.

A tabela a seguir mostra o efeito de variaçóes de ncond para cada $n$. Cada entrada da tabela é média aritmética de 54 testes para $n=5$ e 10 , e 18 testes no caso $n=20$. 


\begin{tabular}{|c|c|c|c|c|}
\hline ncond & $n$ & 5 & 10 & 20 \\
\hline \multirow{4}{*}{0} & tempo total & $1.69 \mathrm{~s}$ & $5 \mathrm{~s}$ & $70 \mathrm{~s}$ \\
& iterações & 29 & 33 & 65 \\
& Hessianas & 214 & 258 & 562 \\
\hline \multirow{4}{*}{3} & tempo total & $1.56 \mathrm{~s}$ & $11 \mathrm{~s}$ & $156 \mathrm{~s}$ \\
& iterações & 30 & 52 & 105 \\
& Hessianas & 214 & 415 & 935 \\
\hline \multirow{4}{*}{6} & tempo total & $2.34 \mathrm{~s}$ & $22 \mathrm{~s}$ & $265 \mathrm{~s}$ \\
& iterações & 40 & 79 & 156 \\
& Hessianas & 286 & 651 & 1437 \\
\hline \multirow{3}{*}{12} & tempo total & $4.75 \mathrm{~s}$ & $51 \mathrm{~s}$ & $572 \mathrm{~s}$ \\
& iterações & 65 & 133 & 257 \\
& Hessianas & 460 & 1095 & 2357 \\
\hline
\end{tabular}

As figuras seguintes mostram a distribuição de tempo e iteraçōes segundo este parâmetro para $n=10$. 
nistribuicao de tempo segundo ncond

Algoritmo sem eliminacoes $n=10$

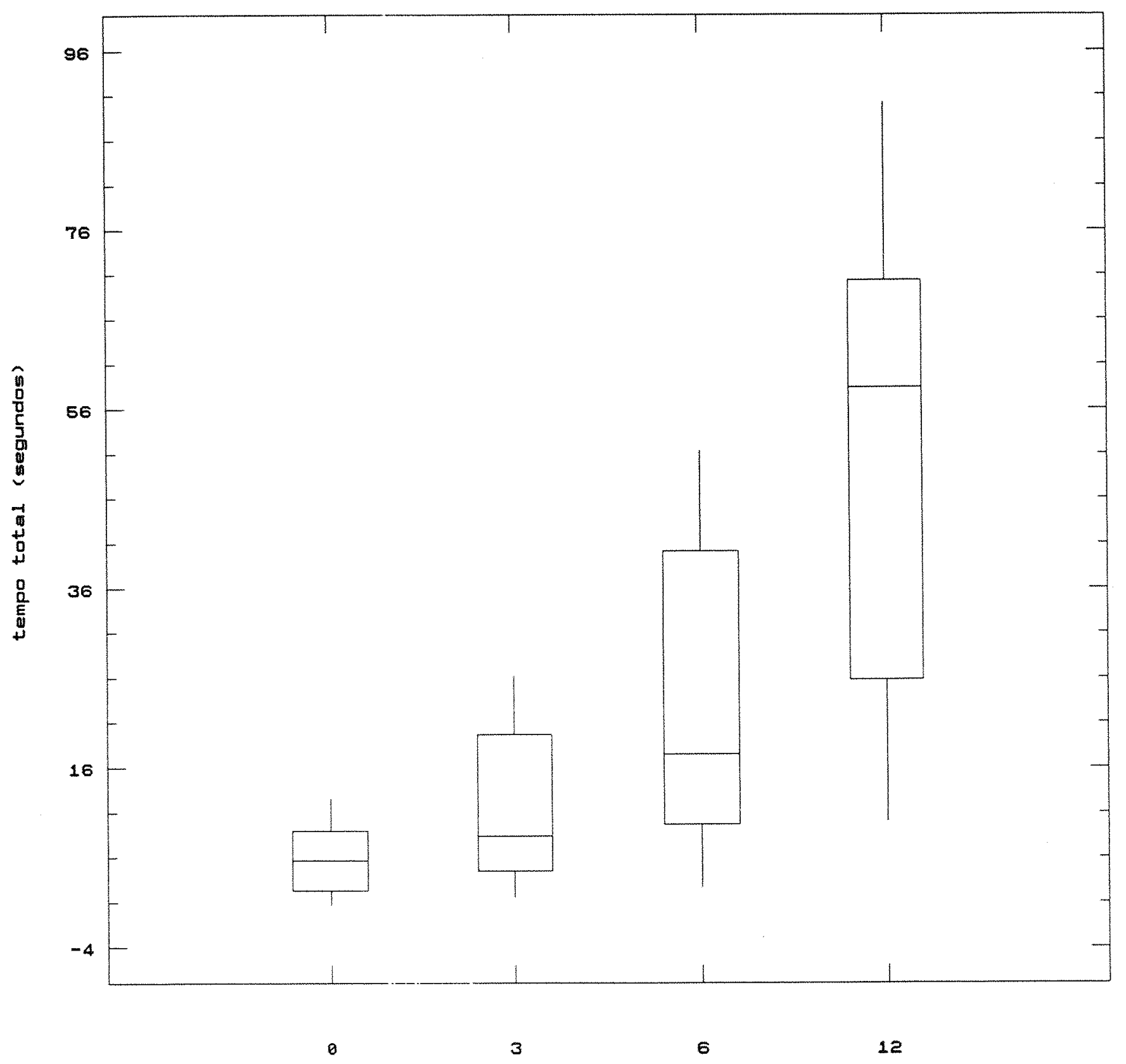

neond 
Distribuicao de \#teracom wando ncond

Algoritmo sem liminacos $n=10$

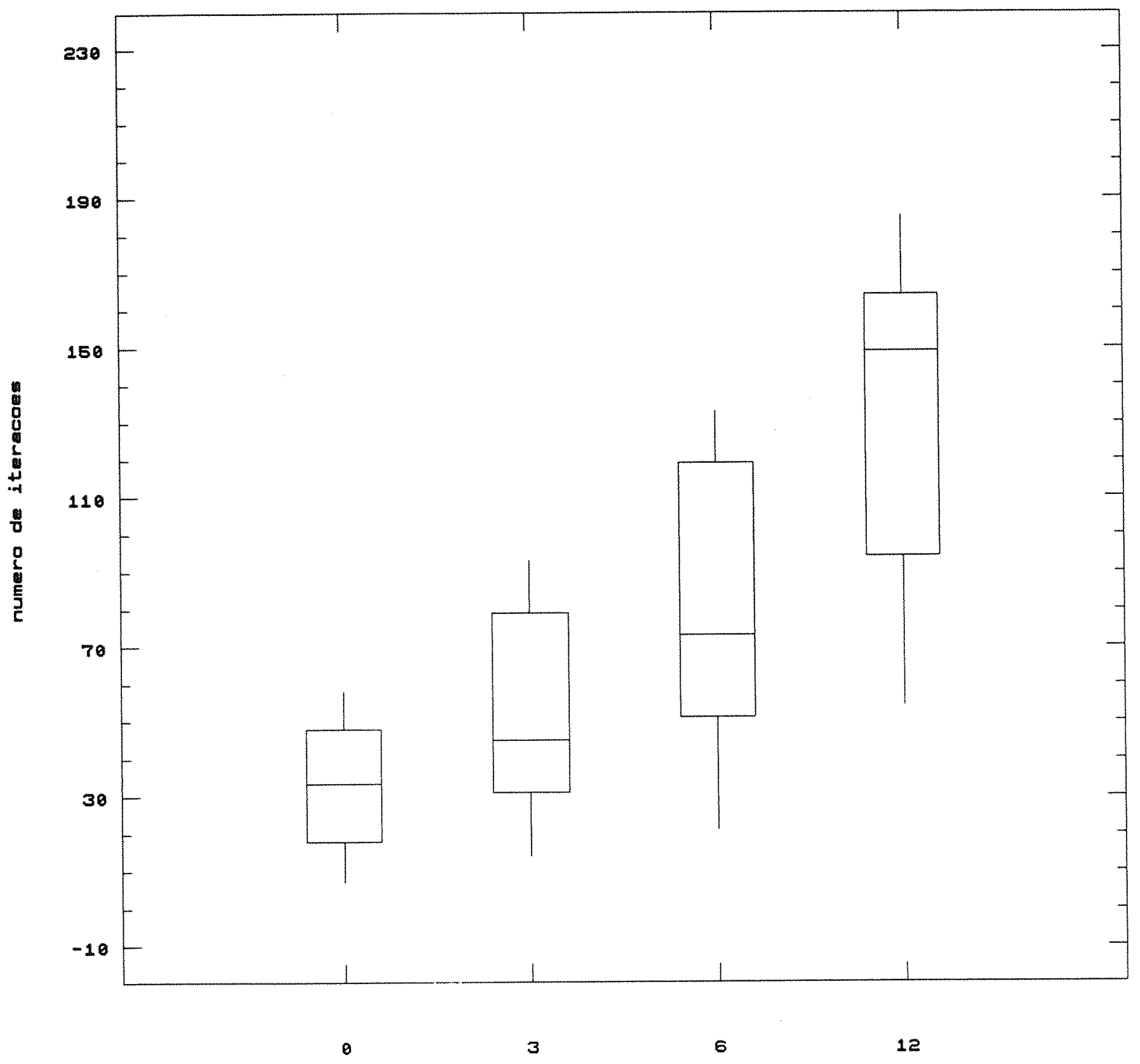

ncond 
Outro parâmetro que influenciou bastante o tempo total e iterações foi a porcentagem de restrições ativas na solução ótima. Ou seja, se a solução ótima tem poucas restrições ativas, o número de iterações e o tempo total são maiores do que quando a solução é um vértice. Além disso, a diferença foi mais acentuada quando $n$ cresce.

A tabela abaixo mostra a média dos resultados para estes valores e os gráficos a seguir mostram a distribuição dos dados.

\begin{tabular}{|c|c|c|c|c|}
\hline na $\left(x^{*}\right)$ & $n$ & 5 & 10 & 20 \\
\hline \multirow{3}{*}{$0.1 n$} & tempo total & $3.71 \mathrm{~s}$ & $39 \mathrm{~s}$ & $534 \mathrm{~s}$ \\
& iteraçōes & 54 & 105 & 228 \\
& Hessianas & 392 & 882 & 2140 \\
\hline \multirow{5}{*}{$0.5 n$} & tempo total & $2.36 \mathrm{~s}$ & $23 \mathrm{~s}$ & $193 \mathrm{~s}$ \\
& iterações & 38 & 74 & 133 \\
& Hessianas & 274 & 598 & 1170 \\
\hline \multirow{3}{*}{$0.9 n$} & tempo total & $1.72 \mathrm{~s}$ & $10 \mathrm{~s}$ & $69 \mathrm{~s}$ \\
& iterações & 31 & 43 & 62 \\
& Hessianas & 214 & 332 & 524 \\
\hline
\end{tabular}


Distribuicao de tempo segundo na ( $\left.x^{x}\right)$

Algoritmo sem eliminacoes $n=20$

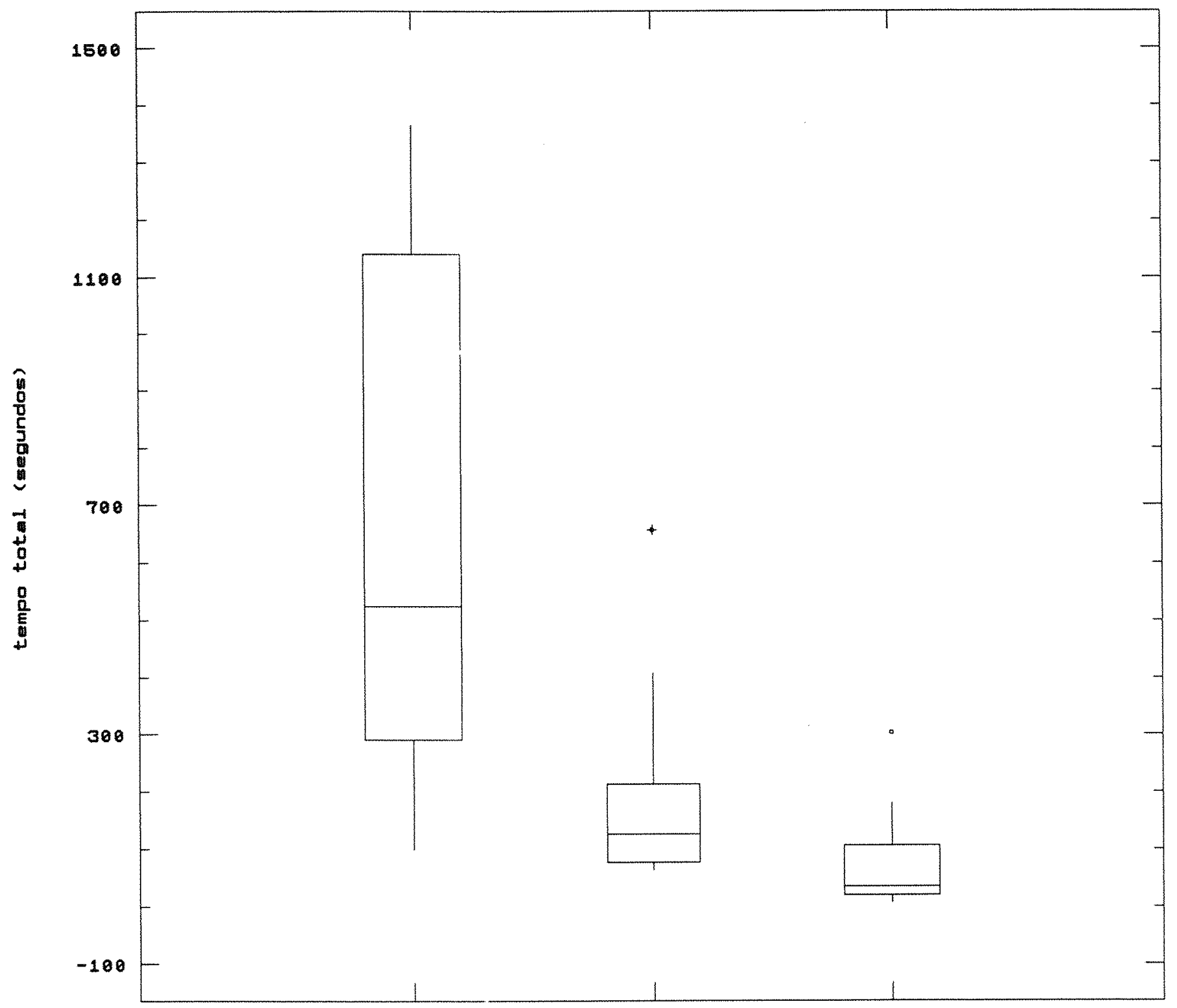

10

58

90

na $\left(x^{*}\right)$ (em \% do numero de restricoe\$) 
Distrib. de \#iteracoes segundo na $\left(x^{*}\right)$

Algoritmo sem eliminacoes $n=20$

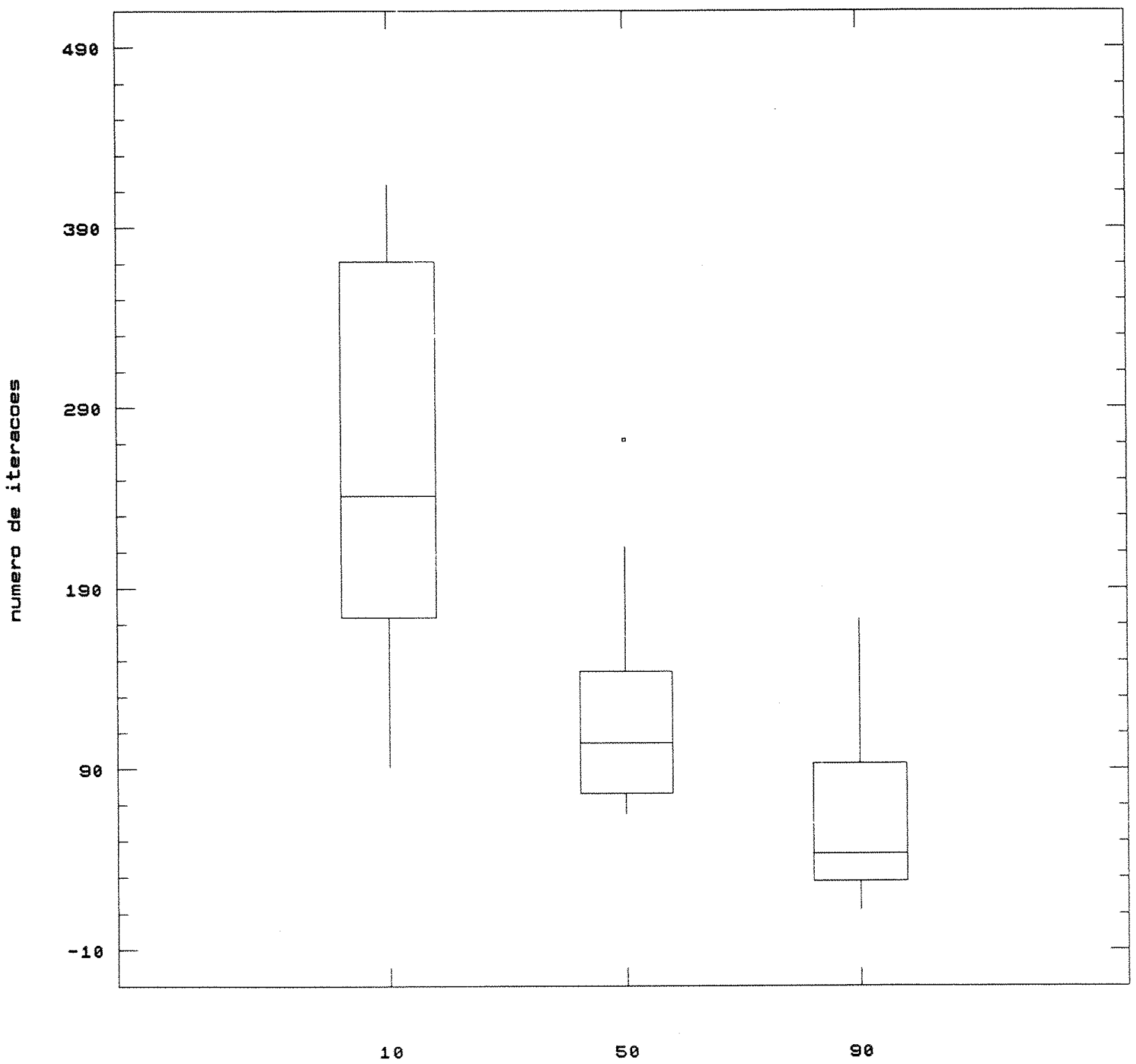

na $\left(x^{*}\right)$ (em \% do numero de restricoes) 
A quantidade de degenerescência dual (ndeg) e o número de restrições quase ativas no ponto inicial $\left(n a\left(x^{0}\right)\right)$ não influenciaram a performance do algoritmo. As tabelas abaixo mostram as médias aritméticas segundo esses parâmetros e os gráficos a seguir mostram a distribuição desses dados.

\begin{tabular}{|c|c|c|c|c|}
\hline$n d e g$ & $n$ & 5 & 10 & 20 \\
\hline & tempo total & $2.61 \mathrm{~s}$ & $24 s$ & $289 \mathrm{~s}$ \\
\hline & erações & 41 & 76 & 148 \\
\hline & Hessianas & 294 & 620 & 1348 \\
\hline \multirow{3}{*}{6} & tempo total & $2.55 \mathrm{~s}$ & $20 \mathrm{~s}$ & $273 \mathrm{~s}$ \\
\hline & iterações & 41 & 71 & 143 \\
\hline & Hessianas & 292 & 579 & 1304 \\
\hline \multirow{3}{*}{12} & tempo total & $2.60 \mathrm{~s}$ & $24 \mathrm{~s}$ & $254 \mathrm{~s}$ \\
\hline & iterações & 41 & 75 & 143 \\
\hline & Hessianas & 295 & 615 & 1291 \\
\hline
\end{tabular}

\begin{tabular}{|c|c|c|c|}
\hline$n a\left(x^{0}\right)$ & $n$ & 5 & 10 \\
\hline \multirow{3}{*}{$0.1 n$} & tempo total & $2.56 \mathrm{~s}$ & $24 \mathrm{~s}$ \\
& iterações & 41 & 74 \\
& Hessianas & 289 & 602 \\
\hline \multirow{3}{*}{$0.5 n$} & tempo total & $2.56 \mathrm{~s}$ & $24 \mathrm{~s}$ \\
& iterações & 41 & 73 \\
& Hessianas & 295 & 599 \\
\hline \multirow{3}{*}{$0.9 n$} & tempo total & $2.60 \mathrm{~s}$ & $24 \mathrm{~s}$ \\
& iterações & 41 & 75 \\
& Hessianas & 296 & 611 \\
\hline
\end{tabular}


Distribuicao de tempo megundo ndeg

Algoritmo sem liminacoes $n=10$

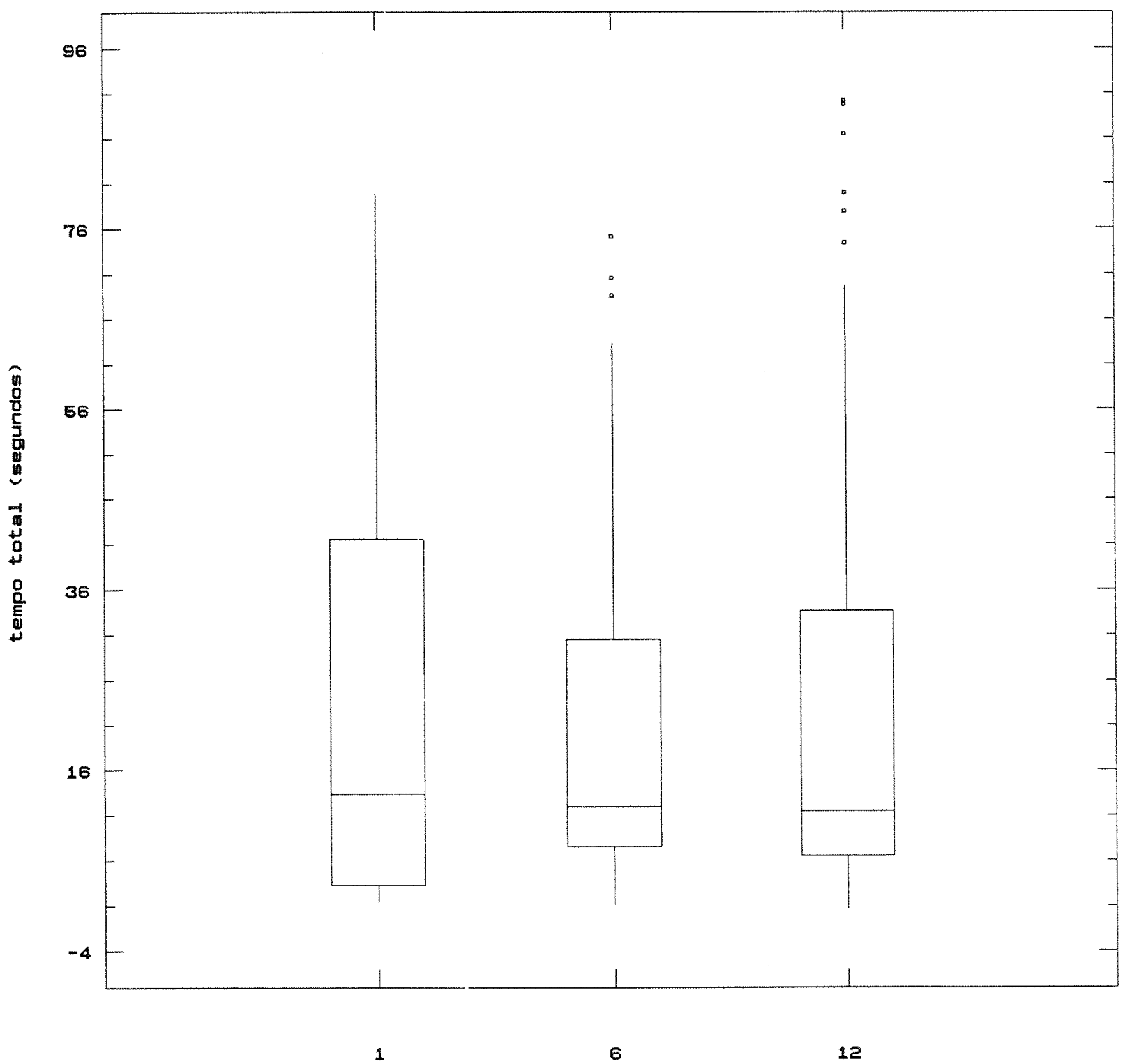

ndeg 


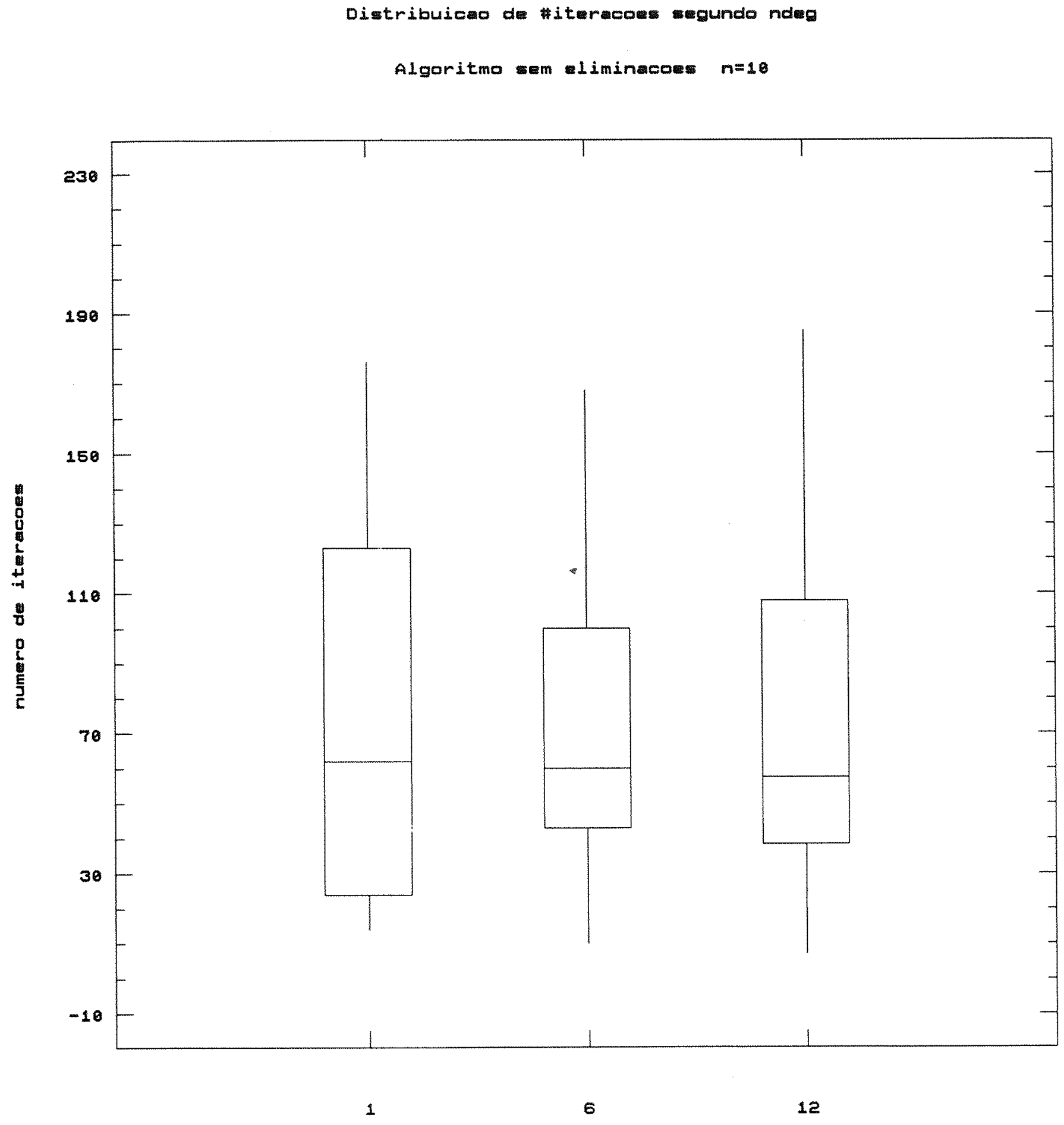

ndeg 
Distribuicao de tempo segundo na $(x 6)$

Algoritmo sem eliminacoem $n=10$

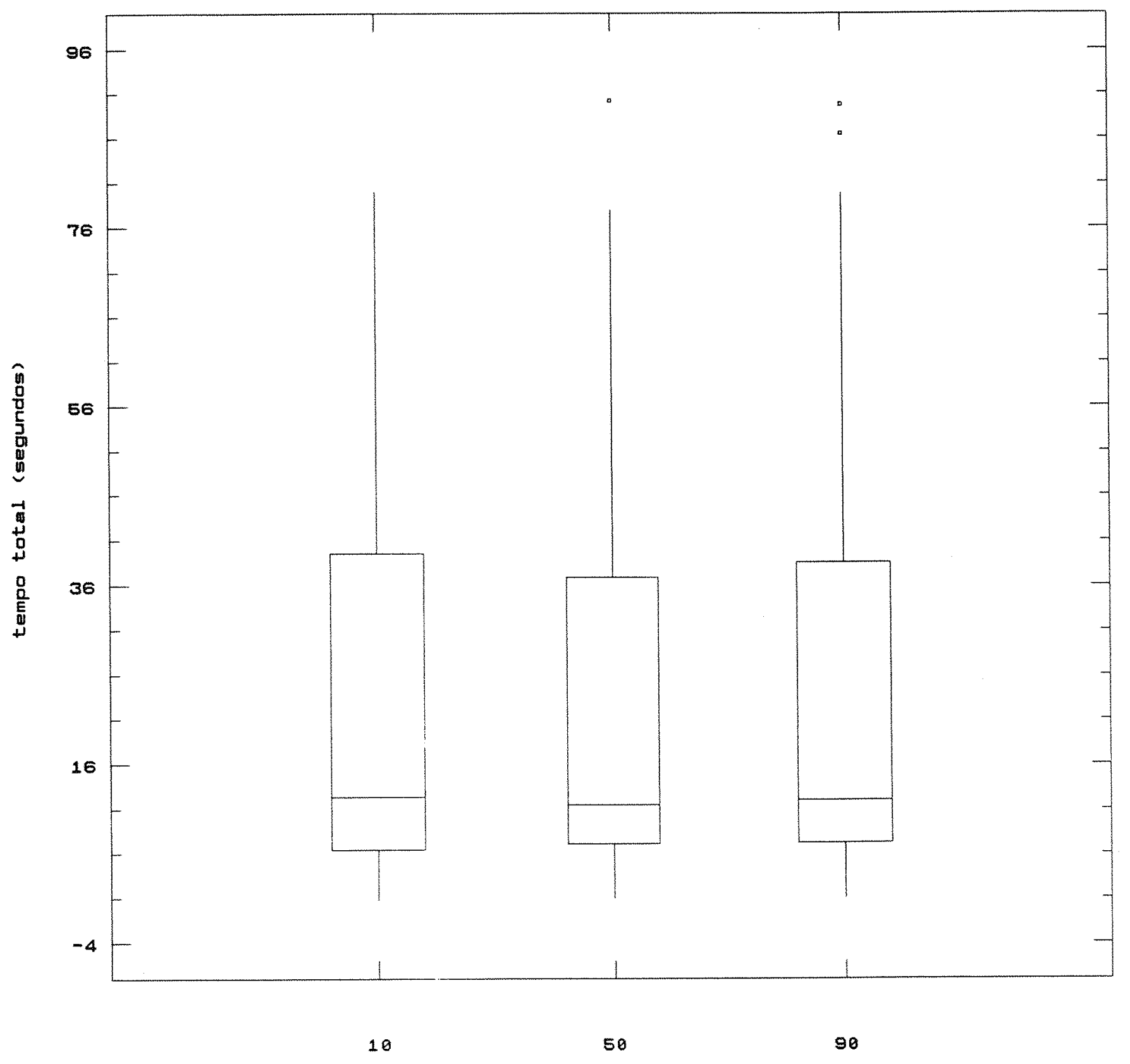

na $(x \theta)$ (em \% do numero de restricoes) 
Distrib. de \#iteracoes moundo na(xi)

Algoritmo sem eliminacoes $n=10$

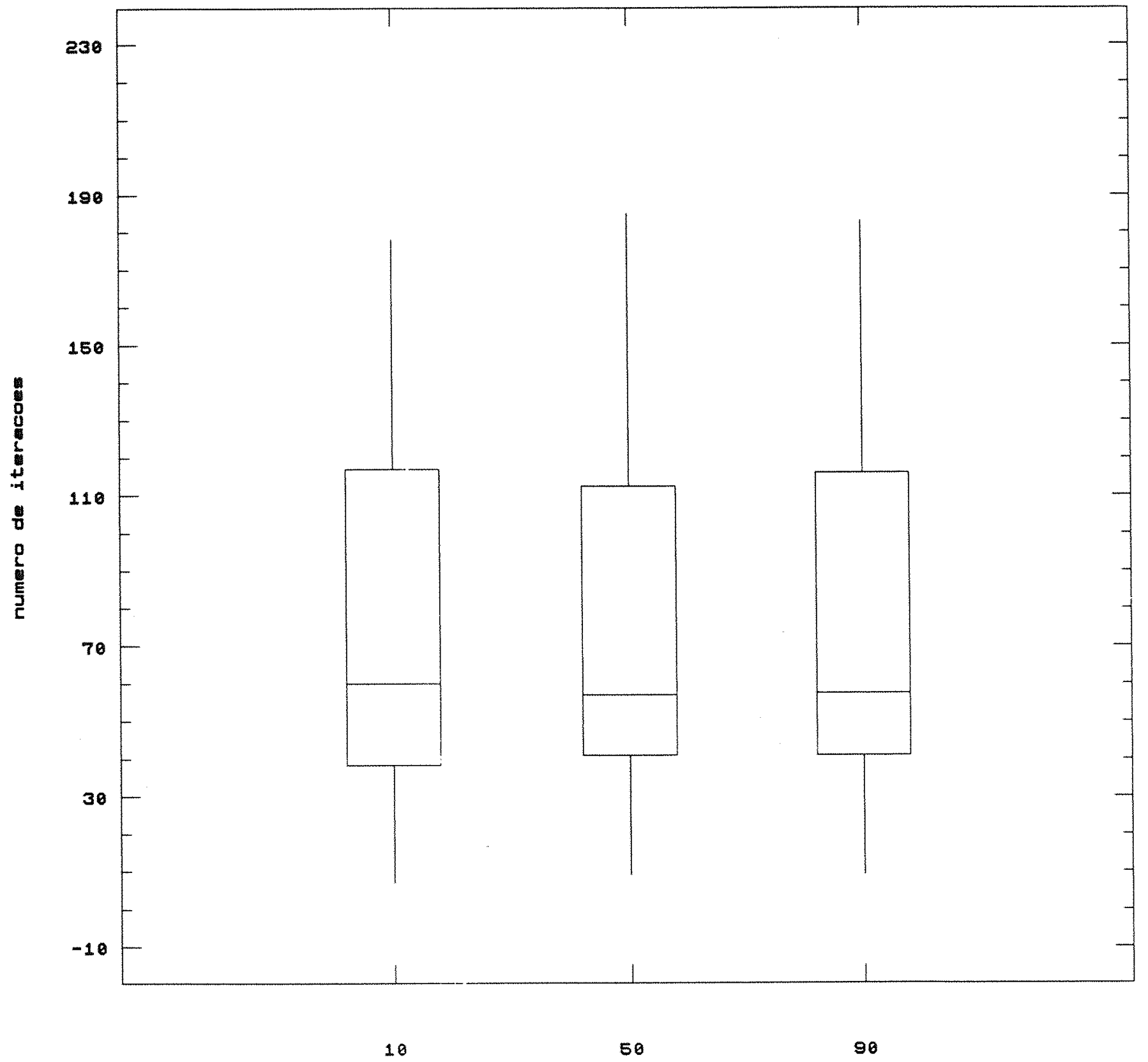

na $(x \theta)$ (em $\%$ do numero de restricoes) 


\subsection{Efeito de eliminação de restrições sobre o algoritmo}

Para cada uma das quatro estratégias escolhidas, testamos 12 variações de modo a analisar a influência do número de restrições mantidas, do passo de eliminação e do tamanho do separador. Variamos estes três parâmetros da seguinte maneira:

Número de restrições mantidas: $n+1,2 n$

Passo de eliminação: $\quad n, 2 n, 4 n$

Tamanho do separador: $\quad d, \frac{d}{n}$

onde $d=\operatorname{dist}\left(\left(x^{k}, y^{k}\right), L\left(x^{k}, y^{k}\right)\right)$

\begin{tabular}{|c|c|c|}
\hline $\begin{array}{c}\text { Critério de escolha de } \\
\text { restriçóes mantidas }\end{array}$ & Método $A$ & Método $A B^{+}$ \\
\hline $\begin{array}{c}\text { Ultimas } \\
\text { restrições }\end{array}$ & Eliminação 1 & Eliminação 3 \\
\hline $\begin{array}{c}\text { Menor valor } \\
\text { de } \varphi(\cdot)\end{array}$ & Eliminação 2 & Eliminação 4 \\
\hline
\end{tabular}


A cada $\mathrm{P}$ iterações eliminaremos restrições em uma iteração $k$ segundo os métodos descritos abaixo:

Método $A$ :

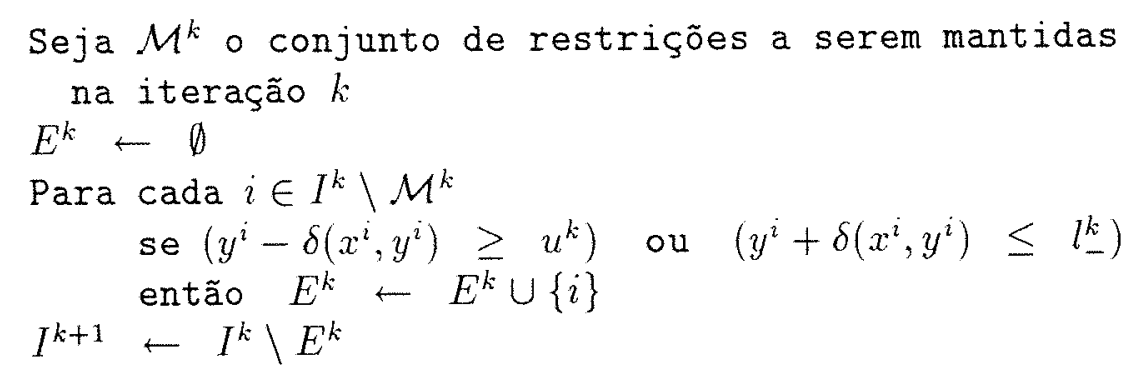

Método $A B^{+}$:

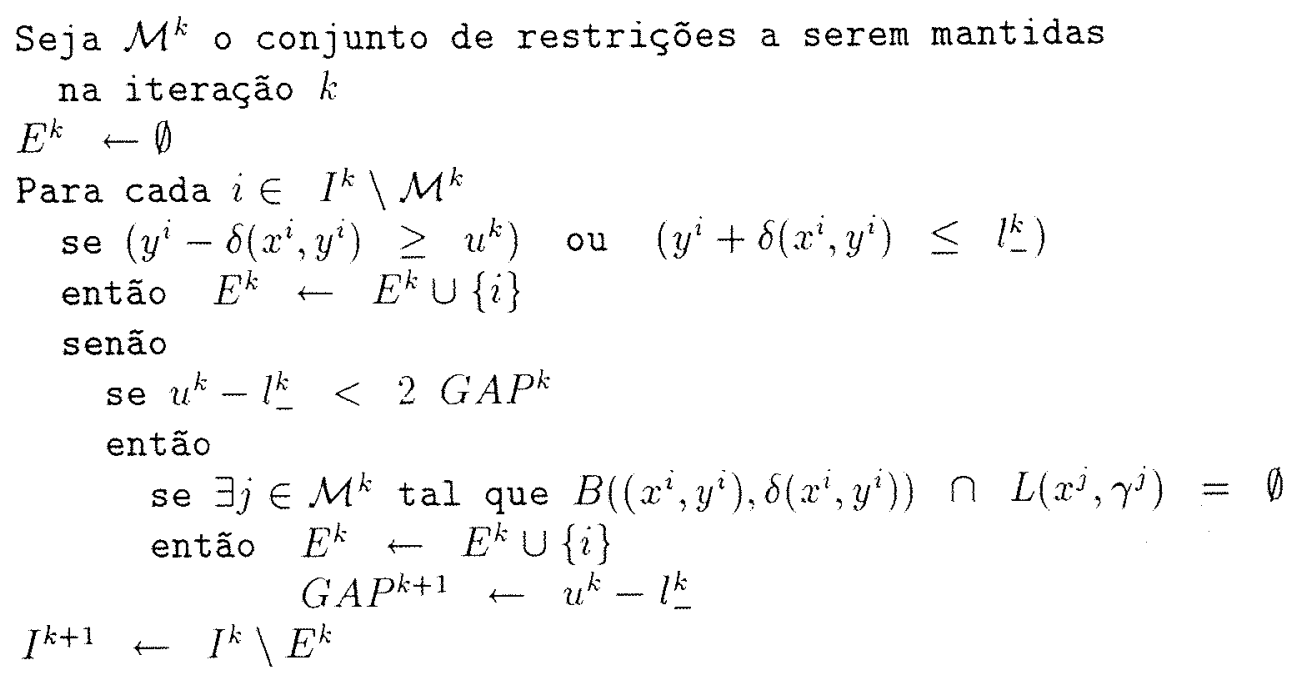


Testamos as variações de cada eliminação para a mesma massa de testes descrita anteriormente. Comparamos as estratégias de eliminação com o algoritmo sem eliminações. As tabelas que apresentaremos mostram a economia média obtida para cada uma das variações. Chamamos de economia média à economia em relação ao tempo médio, ou seja:

$$
\text { Economia média }=\frac{S-E}{S}
$$

onde $S=$ média para uma certa grandeza (tempo, iterações) de todos os dados para o algoritmo sem eliminações

$E=$ média para a mesma grandeza de todos os dados para o algoritmo com a eliminação em questâo

Aqui convém ressaltar que esta medida tende a ser mais afetada por economias em problemas que gastam mais tempo, o que nos pareceu mais interessante do que a média das economias para cada problema.

Os gráficos sob o título "Distribuição de tempo por suas variações" mostram a distribuição dos tempos totais para as variaçôes de uma mesma eliminação, numeradas de 1 até 12 , e o box de número 0 é a distribuição dos tempos para o algoritmo sem eliminações. Esses dados permitem uma visualização global da economia obtida, o que não seria possível apenas com a economia média.

Exibimos também um outro tipo de gráfico que mostra o número da iteração nas abcissas e a quantidade de restrições nas ordenadas. Ele permite visualizar o crescimento do número de restrições ao longo do algoritmo. Selecionamos algumas variaçôes para exibirmos esses gráficos.

\subsubsection{Estratégia de eliminação 1}

As tabelas abaixo mostram as doze variações deste tipo de eliminação para $n=5,10$ e 20, exibindo a economia média de tempo, número de iterações e número total de cálculo de Hessianas. É interessante notar que em alguns casos não houve economia em número de iteraçòes e Hessianas, porém em quase todos houve uma economia de tempo. Os melhores resultados estão marcados com ' $\star$ '. 
$n=5$

\begin{tabular}{|c|c|c|c|c|c|c|c|c|c|}
\hline \multirow{3}{*}{ passo } & \multirow{3}{*}{$\begin{array}{l}\text { tam. } \\
\text { do } \\
\text { sep. }\end{array}$} & \multicolumn{8}{|c|}{ Quantidade de restriçôes mantidas } \\
\hline & & \multicolumn{4}{|c|}{$n+1$} & \multicolumn{4}{|c|}{$2 n$} \\
\hline & & $\#$ & tempo & iter. & Total Hess & $\#$ & tempo & iter. & Total Hess \\
\hline $\mathrm{n}$ & $\bar{d}$ & 1 & $-5 \%$ & $-52 \%$ & $-52 \%$ & 7 & $30 \%$ & $-4 \%$ & $-1 \%$ \\
\hline $\mathrm{n}$ & $\mathrm{d} / \mathrm{n}$ & 2 & $3 \%$ & $-37 \%$ & $-37 \%$ & 8 & $29 \%$ & $0 \%$ & $2 \%$ \\
\hline $2 n$ & $d$ & 3 & $20 \%$ & $-18 \%$ & $-15 \%$ & 9 & $31 \%$ & $0 \%$ & $3 \%$ \\
\hline $2 n$ & $d / n$ & 4 & $24 \%$ & $-10 \%$ & $-7 \%$ & 10 & $28 \%$ & $1 \%$ & $4 \%$ \\
\hline $4 n$ & $d$ & 5 & $24 \%$ & $-7 \%$ & $-4 \%$ & 11 & $27 \%$ & $1 \%$ & $4 \%$ \\
\hline $4 \mathrm{n}$ & $\mathrm{d} / \mathrm{n}$ & 6 & $23 \%$ & $-4 \%$ & $-1 \%$ & 12 & $23 \%$ & $1 \%$ & $4 \%$ \\
\hline
\end{tabular}

$$
n=10
$$

\begin{tabular}{|c|c|c|c|c|c|c|c|c|c|}
\hline \multirow{3}{*}{ passo } & tam. & \multicolumn{6}{|c|}{ Quantidade de restricooes mantidas } \\
\cline { 2 - 9 } & do & \multicolumn{6}{|c|}{$\mathrm{n}+1$} & \multicolumn{3}{|c|}{$2 \mathrm{n}$} \\
\cline { 3 - 9 } & sep. & $\#$ & tempo & iter. & Total Hess & $\#$ & tempo & iter. & Total Hess \\
\hline $\mathrm{n}$ & $\mathrm{d}$ & 1 & $28 \%$ & $-29 \%$ & $-25 \%$ & 7 & $\star 47 \%$ & $7 \%$ & $12 \%$ \\
$\mathrm{n}$ & $\mathrm{d} / \mathrm{n}$ & 2 & $32 \%$ & $-21 \%$ & $-16 \%$ & 8 & $\star 46 \%$ & $7 \%$ & $12 \%$ \\
$2 \mathrm{n}$ & $\mathrm{d}$ & 3 & $\star 45 \%$ & $0 \%$ & $4 \%$ & 9 & $43 \%$ & $6 \%$ & $10 \%$ \\
$2 \mathrm{n}$ & $\mathrm{d} / \mathrm{n}$ & 4 & $\star 45 \%$ & $1 \%$ & $6 \%$ & 10 & $42 \%$ & $6 \%$ & $10 \%$ \\
$4 \mathrm{n}$ & $\mathrm{d}$ & 5 & $39 \%$ & $1 \%$ & $5 \%$ & 11 & $36 \%$ & $5 \%$ & $8 \%$ \\
$4 \mathrm{n}$ & $\mathrm{d} / \mathrm{n}$ & 6 & $39 \%$ & $2 \%$ & $6 \%$ & 12 & $36 \%$ & $5 \%$ & $8 \%$ \\
\hline
\end{tabular}

$$
n=20
$$

\begin{tabular}{|c|c|c|c|c|c|c|c|c|c|}
\hline \multirow{3}{*}{ passo } & \multirow{3}{*}{$\begin{array}{l}\text { tam. } \\
\text { do } \\
\text { sep. }\end{array}$} & \multicolumn{8}{|c|}{ Quantidade de restriçôes mantidas } \\
\hline & & \multicolumn{4}{|c|}{$n+1$} & \multicolumn{4}{|c|}{$2 n$} \\
\hline & & $\#$ & tempo & iter. & Total Hess & $\#$ & tempo & iter. & Total Hess \\
\hline $\mathrm{n}$ & $\mathrm{d}$ & 1 & $37 \%$ & $-13 \%$ & $-8 \%$ & 7 & $\star 46 \%$ & $8 \%$ & $13 \%$ \\
\hline $\mathrm{n}$ & $\mathrm{d} / \mathrm{n}$ & 2 & $42 \%$ & $-3 \%$ & $1 \%$ & 8 & $\star 45 \%$ & $8 \%$ & $13 \%$ \\
\hline $2 \mathrm{n}$ & $\mathrm{d}$ & 3 & $\star 46 \%$ & $3 \%$ & $8 \%$ & 9 & $41 \%$ & $7 \%$ & $11 \%$ \\
\hline $2 n$ & $\mathrm{~d} / \mathrm{n}$ & 4 & $\star 45 \%$ & $4 \%$ & $9 \%$ & 10 & $41 \%$ & $7 \%$ & $10 \%$ \\
\hline $4 n$ & d & 5 & $40 \%$ & $5 \%$ & $9 \%$ & 11 & $36 \%$ & $5 \%$ & $9 \%$ \\
\hline $4 n$ & $\mathrm{~d} / \mathrm{n}$ & 6 & $40 \%$ & $5 \%$ & $9 \%$ & 12 & $35 \%$ & $5 \%$ & $8 \%$ \\
\hline
\end{tabular}


Podemos notar que manter $2 n$ restrições e utilizar passo $n$ foi um pouco melhor que manter $n+1$ restrições utilizando passo $2 n$. Os piores resultados foram obtidos mantendo $n+1$ restrições e utilizando passo $n$. O tamanho do separador não afetou muito os resultados. A seguir exibimos os gráficos da distribuição para as diferentes variaçôes. $O$ box de número 0 mostra $o$ algoritmo sem eliminações. Os boxes relativos às variações 1 e 2 mostram que foram as piores para os três valores de $n$.

A seguir podemos ver os gráficos da distribuiçâo do número de restrições por iteração. Para a melhor variação obtida (manter $2 n$ restrições com passo $n$ ), em todos os testes com $n=10$ e 20 obtivemos eliminação máxima, ou seja, exatamente $2 n$ restrições foram mantidas. Isto quer dizer que os demais pontos satisfizeram o critério de eliminação. A pior variação (mantendo $n+1$ restrições com passo $n$ ) teve um crescimento menos regular do número de restrições e, apesar de exigir manutenção mínima de de apenas $n+1$ restrições medianamente manteve aproximadamente $2 n$, e os maiores valores chegaram a $4 n$ restriçôes. Além disso, os exemplos mais longos gastaram aproximadamente o dobro em iterações.

A conclusão que podemos tirar sobre este efeito é que eliminar restrições demais fez com que o algoritmo perdesse a aproximação da epígrafe que havia obtido, fazendo com que o critério de eliminaçào nào fosse obedecido pelas outras restriçôes em iteraçòes subseqüentes e, além disso, demorando mais a convergir. 
Eliminacao $1 \quad(n=10)$

Distribuican de tempo por suas variacoes

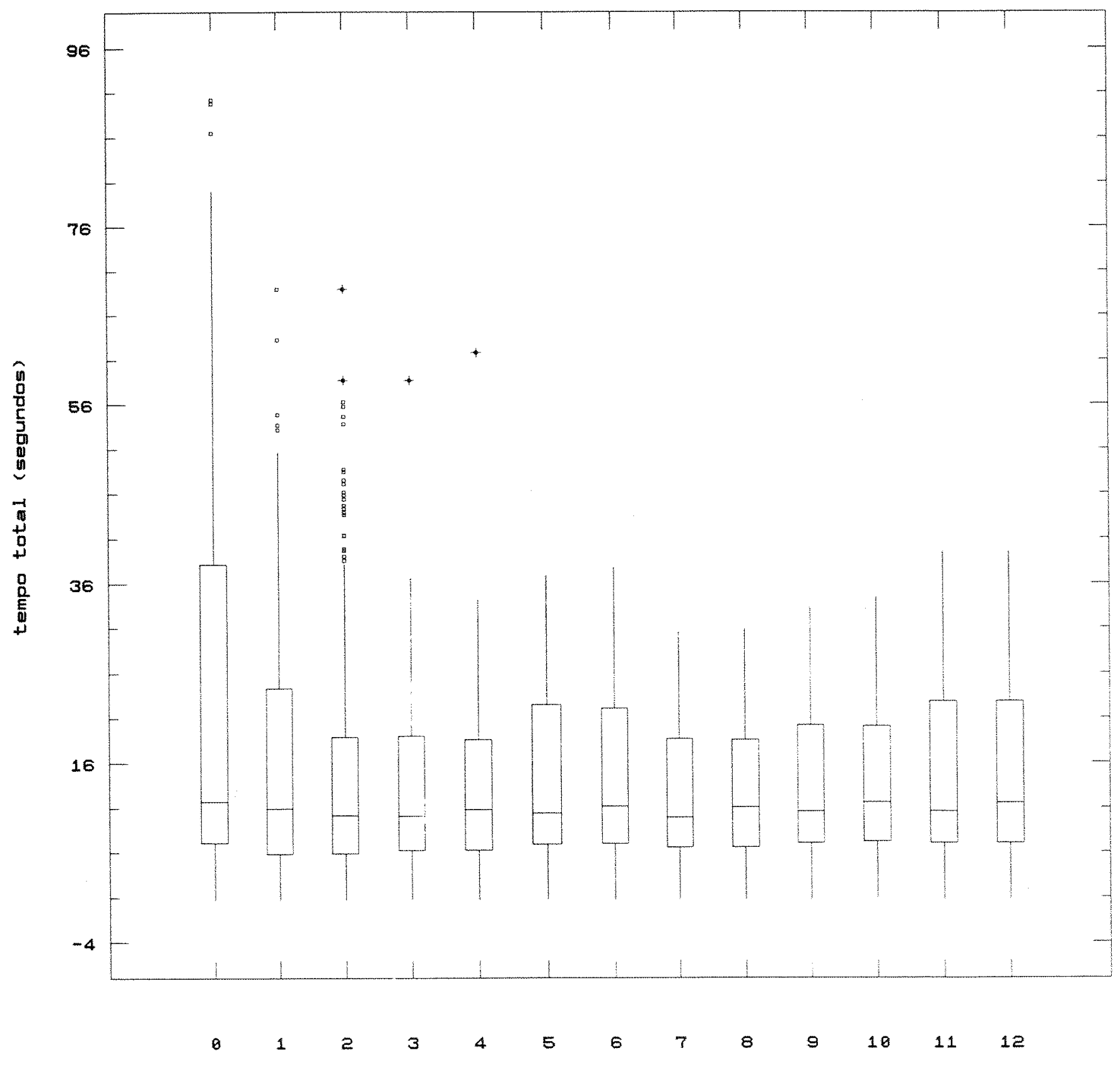

variacoes 
Eliminacac $1 \quad(n=10)$

Mantem $2 n$ restricom, pis

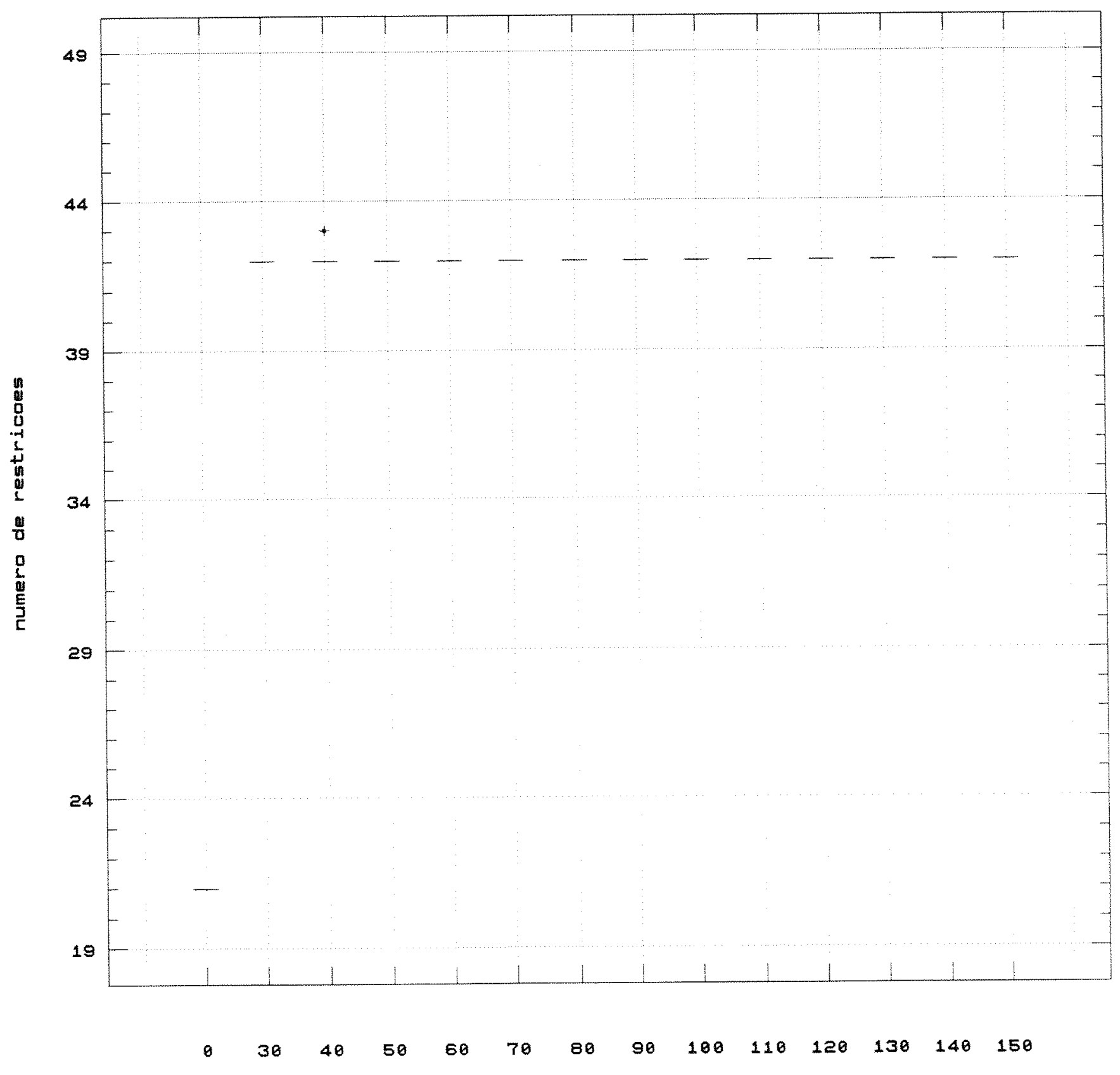

iteracam 
Eliminacao 1 ( $(n=10)$

Mantem $n+1$ restricow, pases $n$

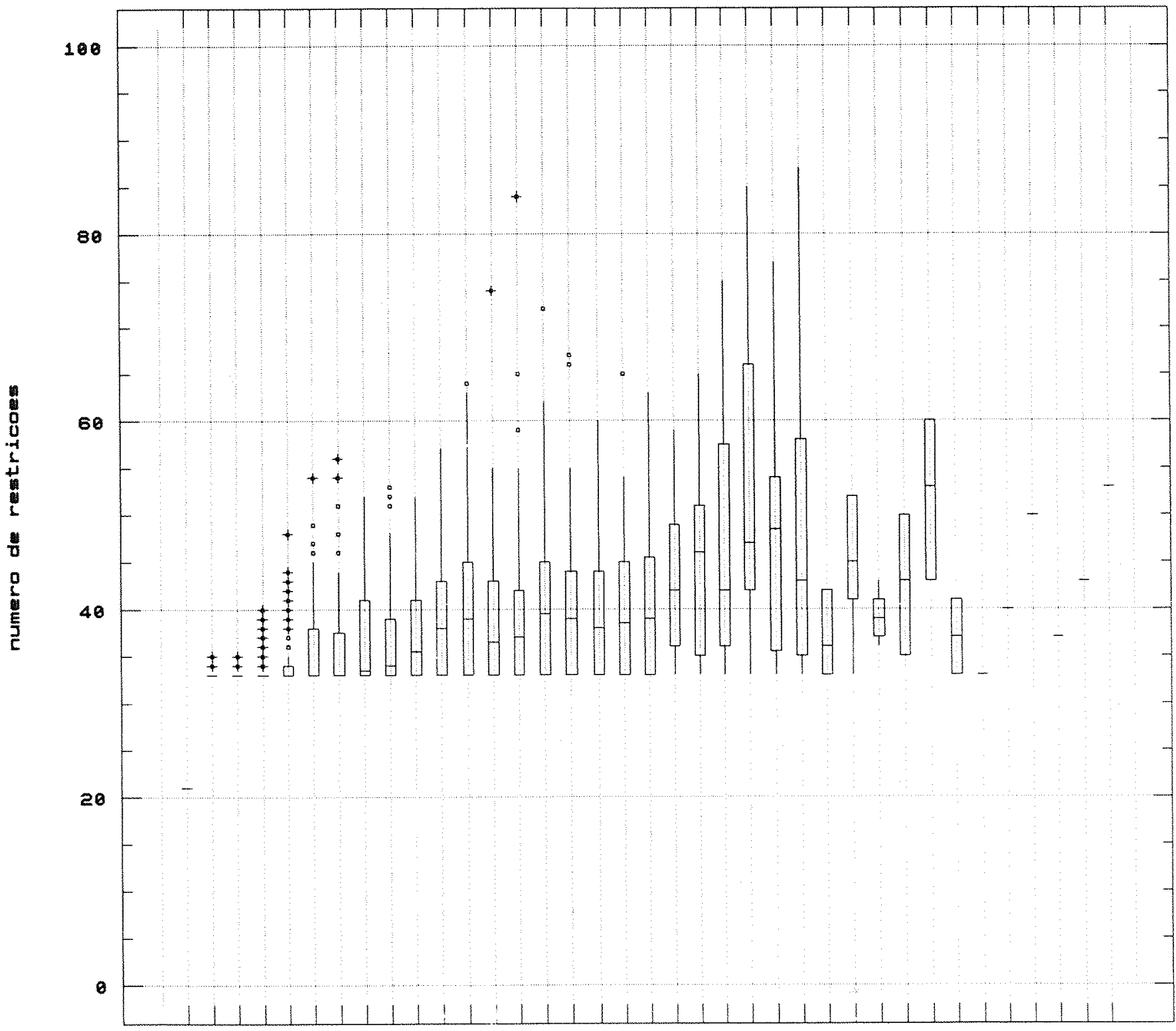

$\begin{array}{llllllllllllllllll}30 & 50 & 70 & 90 & 110 & 130 & 150 & 170 & 190 & 210 & 230 & 250 & 270 & 290 & 310 & 330 & 350 & 370\end{array}$

$20 \quad 40 \quad 60 \quad 80 \quad 100 \quad 120 \quad 140 \quad 160 \quad 180 \quad 200 \quad 220 \quad 240 \quad 260 \quad 280 \quad 300 \quad 320 \quad 340 \quad 360$ 


\subsubsection{Estratégia de eliminação 2}

A seguir temos as tabelas de economia de tempo para cada valor de $n$.

$$
n=5
$$

\begin{tabular}{|c|c|c|c|c|c|c|c|c|c|}
\hline \multirow{3}{*}{ passo } & tam. & \multicolumn{6}{|c|}{ Quantidade de restriçōes mantidas } \\
\cline { 3 - 9 } & do & \multicolumn{6}{|c|}{$\mathrm{n}+1$} & \multicolumn{4}{|c|}{$2 \mathrm{n}$} \\
\cline { 3 - 9 } & sep. & $\#$ & tempo & iter. & Total Hess & $\#$ & tempo & iter. & Total Hess \\
\hline $\mathrm{n}$ & $\mathrm{d}$ & 1 & $\star 40 \%$ & $0 \%$ & $4 \%$ & 7 & $\star 39 \%$ & $4 \%$ & $8 \%$ \\
$\mathrm{n}$ & $\mathrm{d} / \mathrm{n}$ & 2 & $\star 36 \%$ & $0 \%$ & $4 \%$ & 8 & $\star 33 \%$ & $4 \%$ & $7 \%$ \\
$2 \mathrm{n}$ & $\mathrm{d}$ & 3 & $39 \%$ & $1 \%$ & $6 \%$ & 9 & $34 \%$ & $4 \%$ & $7 \%$ \\
$2 \mathrm{n}$ & $\mathrm{d} / \mathrm{n}$ & 4 & $34 \%$ & $2 \%$ & $6 \%$ & 10 & $30 \%$ & $3 \%$ & $6 \%$ \\
$4 \mathrm{n}$ & $\mathrm{d}$ & 5 & $32 \%$ & $2 \%$ & $5 \%$ & 11 & $28 \%$ & $3 \%$ & $6 \%$ \\
$4 \mathrm{n}$ & $\mathrm{d} / \mathrm{n}$ & 6 & $28 \%$ & $2 \%$ & $4 \%$ & 12 & $24 \%$ & $2 \%$ & $5 \%$ \\
\hline
\end{tabular}

$$
n=10
$$

\begin{tabular}{|c|c|c|c|c|c|c|c|c|c|}
\hline \multirow{3}{*}{ passo } & \multirow{3}{*}{$\begin{array}{c}\text { tam. } \\
\text { do } \\
\text { sep. }\end{array}$} & \multicolumn{8}{|c|}{ Quantidade de restriçòes mantidas } \\
\hline & & \multicolumn{4}{|c|}{$\mathrm{n}+1$} & \multicolumn{4}{|c|}{$2 n$} \\
\hline & & \# & tempo & iter. & Total Hess & \# & tempo & iter. & Total Hess \\
\hline $\mathrm{n}$ & $\mathrm{d}$ & 1 & $\star 53 \%$ & $5 \%$ & $10 \%$ & 7 & $\star 47 \%$ & $8 \%$ & $12 \%$ \\
\hline $\mathrm{n}$ & $\mathrm{d} / \mathrm{n}$ & 2 & $\star 52 \%$ & $6 \%$ & $11 \%$ & 8 & $\star 47 \%$ & $8 \%$ & $12 \%$ \\
\hline $2 \mathrm{n}$ & $\mathrm{d}$ & 3 & $50 \%$ & $6 \%$ & $12 \%$ & 9 & $43 \%$ & $7 \%$ & $10 \%$ \\
\hline $2 \mathrm{n}$ & $\mathrm{d} / \mathrm{n}$ & 4 & $49 \%$ & $7 \%$ & $12 \%$ & 10 & $42 \%$ & $6 \%$ & $10 \%$ \\
\hline $4 \mathrm{n}$ & d & 5 & $42 \%$ & $5 \%$ & $9 \%$ & 11 & $37 \%$ & $5 \%$ & $8 \%$ \\
\hline $4 \mathrm{n}$ & $\mathrm{d} / \mathrm{n}$ & 6 & $42 \%$ & $6 \%$ & $10 \%$ & 12 & $36 \%$ & $5 \%$ & $8 \%$ \\
\hline
\end{tabular}


$n=20$

\begin{tabular}{|c|c|c|c|c|c|c|c|c|c|}
\hline \multirow{3}{*}{ passo } & tam. & \multicolumn{6}{|c|}{ Quantidade de restriçóes mantidas } \\
\cline { 2 - 9 } & do & \multicolumn{3}{|c|}{$\mathrm{n}+1$} & \multicolumn{4}{|c|}{$2 \mathrm{n}$} \\
\cline { 3 - 9 } & sep. & $\#$ & tempo & iter. & Total Hess & $\#$ & tempo & iter. & Total Hess \\
\hline $\mathrm{n}$ & $\mathrm{d}$ & 1 & $\star 49 \%$ & $4 \%$ & $10 \%$ & 7 & $\star 45 \%$ & $8 \%$ & $13 \%$ \\
$\mathrm{n}$ & $\mathrm{d} / \mathrm{n}$ & 2 & $\star 49 \%$ & $6 \%$ & $11 \%$ & 8 & $\star 45 \%$ & $8 \%$ & $13 \%$ \\
$2 \mathrm{n}$ & $\mathrm{d}$ & 3 & $48 \%$ & $6 \%$ & $12 \%$ & 9 & $41 \%$ & $7 \%$ & $11 \%$ \\
$2 \mathrm{n}$ & $\mathrm{d} / \mathrm{n}$ & 4 & $47 \%$ & $7 \%$ & $12 \%$ & 10 & $41 \%$ & $7 \%$ & $11 \%$ \\
$4 \mathrm{n}$ & $\mathrm{d}$ & 5 & $41 \%$ & $6 \%$ & $10 \%$ & 11 & $36 \%$ & $6 \%$ & $9 \%$ \\
$4 \mathrm{n}$ & $\mathrm{d} / \mathrm{n}$ & 6 & $40 \%$ & $6 \%$ & $10 \%$ & 12 & $36 \%$ & $6 \%$ & $9 \%$ \\
\hline
\end{tabular}

Podemos notar que o menor passo de eliminação $(n)$ com a manutenção mínima de $n+1$ restrições foi a variaçâo que deu melhores resultados (variação 1). Além disso, esta variação foi melhor em termos de economia de tempo que todas as variaçôes para a estratégia de eliminação 1 . Podemos então concluir que as restriçòes associadas a pontos com menor valor de $\varphi(\cdot)$ foram as que melhor definiram o poliedro de restrições.

Selecionamos as duas melhores variaçòes ( 1 e 7 ) para exibirmos os gráficos de restrições por iteração. Aqui também ocorreu que, quando mantemos $n+1$ restriçôes no mínimo, há um efeito de oscilação maior no número de restrições do que quando mantemos $2 n$ restrições, onde a eliminação atinge a máxima permitida para todas as iterações. Porém, foi mais vantajosa a manutenção de $n+1$ restrições, pois a oscilaçào de restrições esteve quase sempre abaixo de $2 n$. O número de iteraçōes máximo foi comparável. 
Eliminacao $2(n=20)$

Distribuicao de tempo por suas variacoes

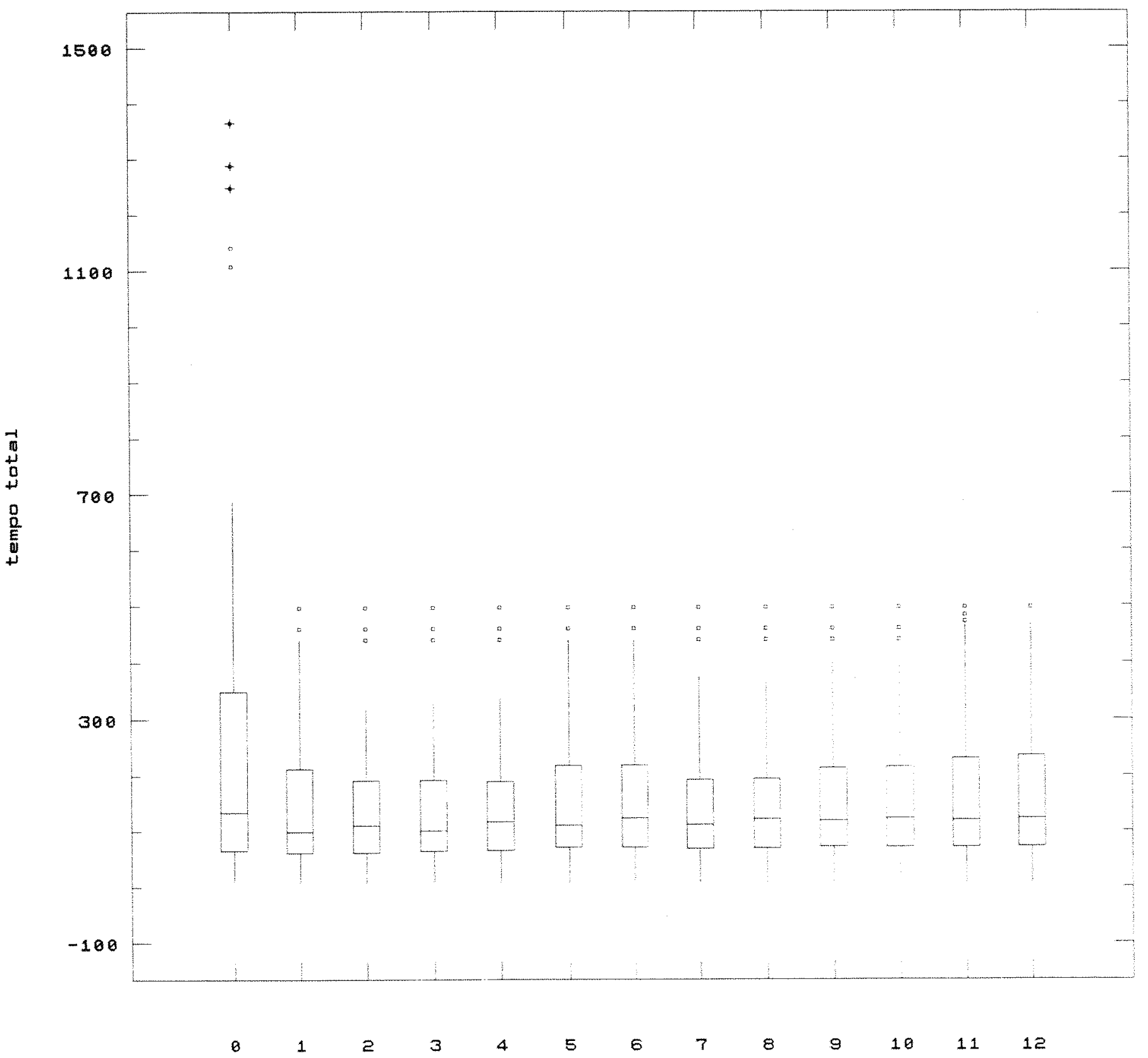

variacoes 
Eliminacao $2 \quad(n=28)$

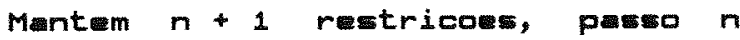

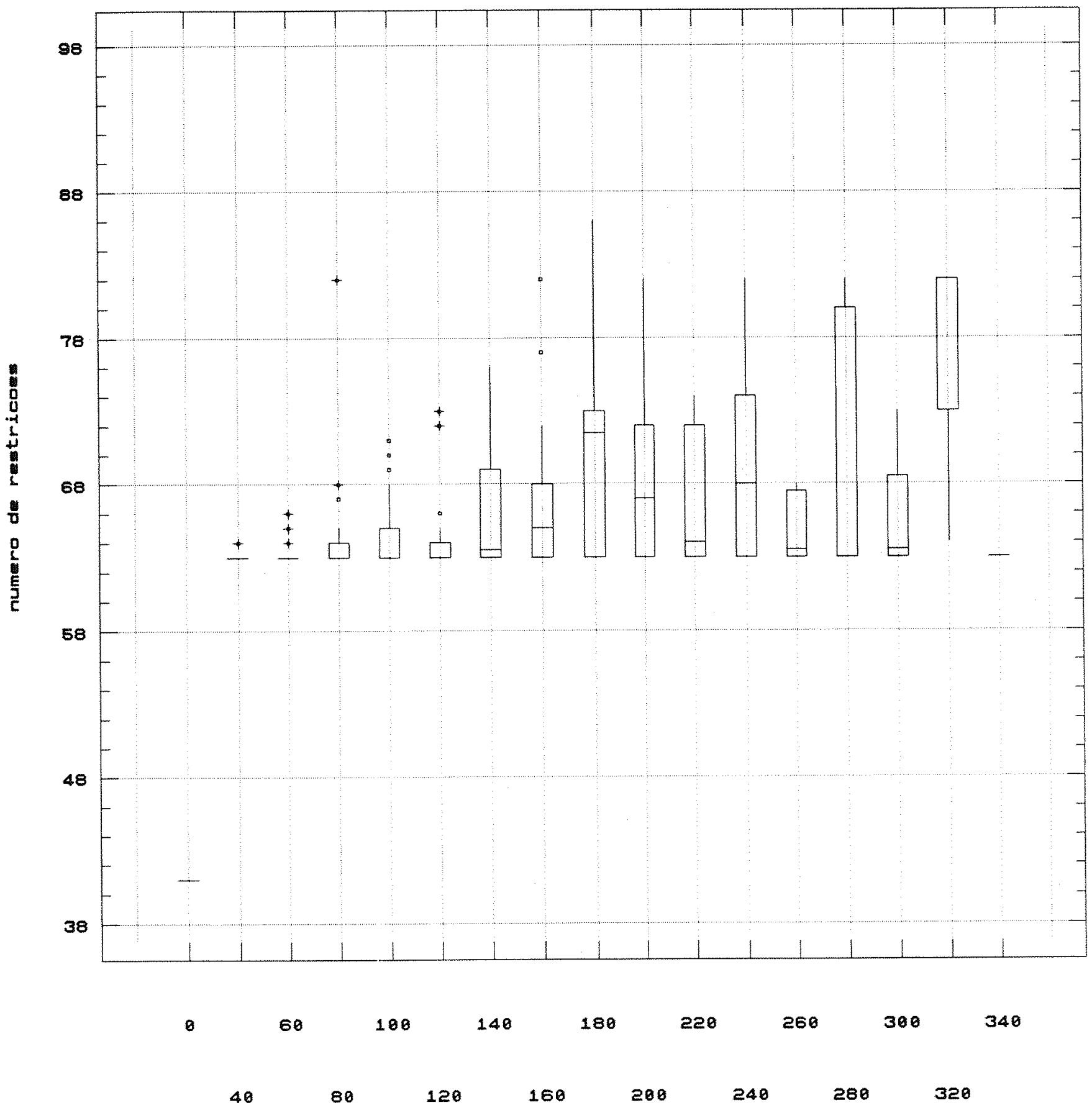

iteracoes 
Eliminacao $2 \quad(n=28)$

Mantem $2 n$ restricoes, passo $n$

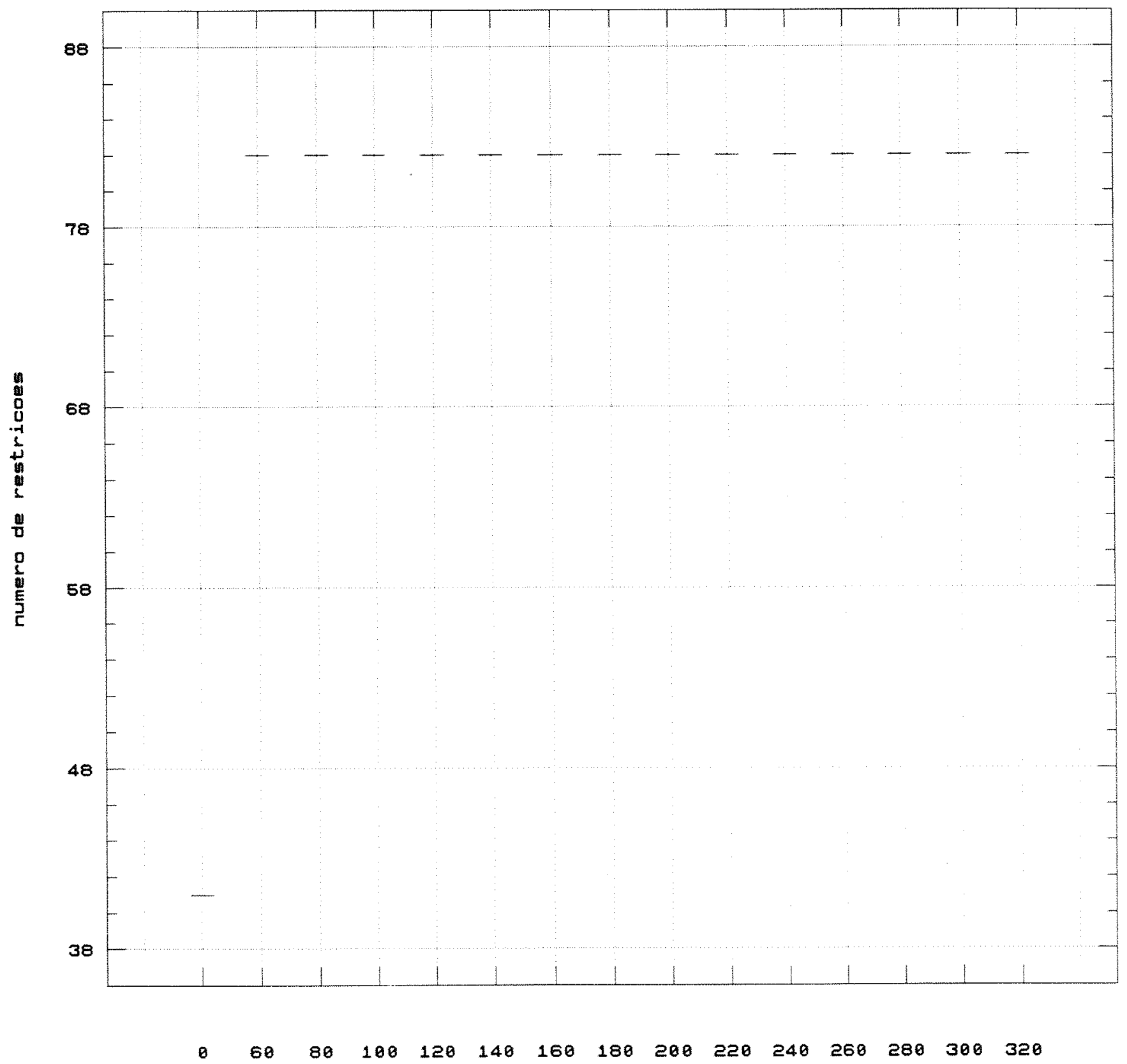

iteracoes 


\subsubsection{Estratégia de eliminação 3}

A diferença desta estratégia para a eliminação 1 é que permitimos mais eliminações toda vez que há um decréscimo suficiente de $\left(u^{k}-l_{-}^{k}\right)$. Pela observação das tabelas de economia, vemos que os melhores resultados foram obtidos com $n=10$. Outro ponto notável é que para os três valores de $n$ apareceram aumentos consideráveis de tempo em relação ao caso sem eliminação, nos casos que mantêm $n+1$ restrições e passo $n$. Esses aumentos de tempo estão associados a um aumento no número de iterações para chegar na convergência. Também podemos observar que manter $2 n$ restrições foi sensivelmente melhor que manter $n+1$.

Os melhores resultados obtidos são comparáveis à estratégia de eliminação 1. Com base nestas considerações, podemos dizer eliminação 1 mostrou-se mais eficiente.

$$
n=5
$$

\begin{tabular}{|c|c|c|c|c|c|c|c|c|c|}
\hline \multirow{3}{*}{ passo } & tam. & \multicolumn{6}{|c|}{ Quantidade de restriçóes mantidas } \\
\cline { 3 - 9 } & sep. & \# & tempo & iter. & Total Hess & $\#$ & tempo & iter. & Total Hess \\
\cline { 3 - 9 } & $\mathrm{d}$ & 1 & $-530 \%$ & $-866 \%$ & $-976 \%$ & 7 & $-49 \%$ & $-118 \%$ & $-123 \%$ \\
$\mathrm{n}$ & $\mathrm{d} / \mathrm{n}$ & 2 & $-530 \%$ & $-845 \%$ & $-956 \%$ & 8 & $-50 \%$ & $-107 \%$ & $-113 \%$ \\
$\mathrm{n}$ & $\mathrm{d}$ & 3 & $-116 \%$ & $-223 \%$ & $-239 \%$ & 9 & $-37 \%$ & $-87 \%$ & $-93 \%$ \\
$2 \mathrm{n}$ & $\mathrm{d} / \mathrm{n}$ & 4 & $-120 \%$ & $-217 \%$ & $-234 \%$ & 10 & $-41 \%$ & $-85 \%$ & $-91 \%$ \\
$4 \mathrm{n}$ & $\mathrm{d}$ & 5 & $25 \%$ & $-9 \%$ & $-6 \%$ & 11 & $\star 25 \%$ & $0 \%$ & $0 \%$ \\
$4 \mathrm{n}$ & $\mathrm{d} / \mathrm{n}$ & 6 & $\star 26 \%$ & $-8 \%$ & $-5 \%$ & 12 & $24 \%$ & $-1 \%$ & $0 \%$ \\
\hline
\end{tabular}




$$
n=10
$$

\begin{tabular}{|c|c|c|c|c|c|c|c|c|c|}
\hline \multirow{3}{*}{ passo } & \multirow{3}{*}{$\begin{array}{c}\text { tam. } \\
\text { do } \\
\text { sep. }\end{array}$} & \multicolumn{8}{|c|}{ Quantidade de restrições mantidas } \\
\hline & & \multicolumn{4}{|c|}{$n+1$} & \multicolumn{4}{|c|}{$2 n$} \\
\hline & & $\#$ & tempo & iter. & Total Hess & $\#$ & tempo & iter. & Total Hess \\
\hline $\mathrm{n}$ & $\mathrm{d}$ & 1 & $-211 \%$ & $-446 \%$ & $-489 \%$ & 7 & $\star 47 \%$ & $8 \%$ & $12 \%$ \\
\hline $\mathrm{n}$ & $\mathrm{d} / \mathrm{n}$ & 2 & $-209 \%$ & $-440 \%$ & $-484 \%$ & 8 & $\star 47 \%$ & $8 \%$ & $12 \%$ \\
\hline $2 n$ & $\mathrm{~d}$ & 3 & $31 \%$ & $-23 \%$ & $-20 \%$ & 9 & $43 \%$ & $7 \%$ & $11 \%$ \\
\hline $2 n$ & $\mathrm{~d} / \mathrm{n}$ & 4 & $31 \%$ & $-23 \%$ & $-20 \%$ & 10 & $43 \%$ & $7 \%$ & $11 \%$ \\
\hline $4 \mathrm{n}$ & d & 5 & $\star 39 \%$ & $0 \%$ & $4 \%$ & 11 & $37 \%$ & $5 \%$ & $8 \%$ \\
\hline $4 n$ & $\mathrm{~d} / \mathrm{n}$ & 6 & $\star 39 \%$ & $0 \%$ & $4 \%$ & 12 & $37 \%$ & $5 \%$ & $8 \%$ \\
\hline
\end{tabular}

$$
n=20
$$

\begin{tabular}{|c|c|c|c|c|c|c|c|c|c|}
\hline \multirow{3}{*}{ passo } & \multirow{3}{*}{$\begin{array}{l}\text { tam. } \\
\text { do } \\
\text { sep. }\end{array}$} & \multicolumn{8}{|c|}{ Quantidade de restriçôes mantidas } \\
\hline & & \multicolumn{4}{|c|}{$n+1$} & \multicolumn{4}{|c|}{$2 n$} \\
\hline & & $\#$ & tempo & iter. & Total Hess & $\#$ & tempo & iter. & Total Hess \\
\hline $\mathrm{n}$ & $\mathrm{d}$ & 1 & $-59 \%$ & $-171 \%$ & $-176 \%$ & 7 & $\star 43 \%$ & $8 \%$ & $12 \%$ \\
\hline $\mathrm{n}$ & $\mathrm{d} / \mathrm{n}$ & 2 & $-59 \%$ & $-171 \%$ & $-176 \%$ & 8 & $\star 43 \%$ & $8 \%$ & $12 \%$ \\
\hline $2 n$ & d & 3 & $\star 44 \%$ & $3 \%$ & $8 \%$ & 9 & $37 \%$ & $6 \%$ & $10 \%$ \\
\hline $2 \mathrm{n}$ & $\mathrm{d} / \mathrm{n}$ & 4 & $\star 44 \%$ & $3 \%$ & $8 \%$ & 10 & $37 \%$ & $6 \%$ & $10 \%$ \\
\hline $4 n$ & d & 5 & $37 \%$ & $3 \%$ & $7 \%$ & 11 & $32 \%$ & $5 \%$ & $8 \%$ \\
\hline $4 n$ & $\mathrm{~d} / \mathrm{n}$ & 6 & $37 \%$ & $4 \%$ & $7 \%$ & 12 & $32 \%$ & $5 \%$ & $8 \%$ \\
\hline
\end{tabular}




$$
\text { Eliminacan } 3 \quad(n=5)
$$

Distribuican de tempo por suas variacoes

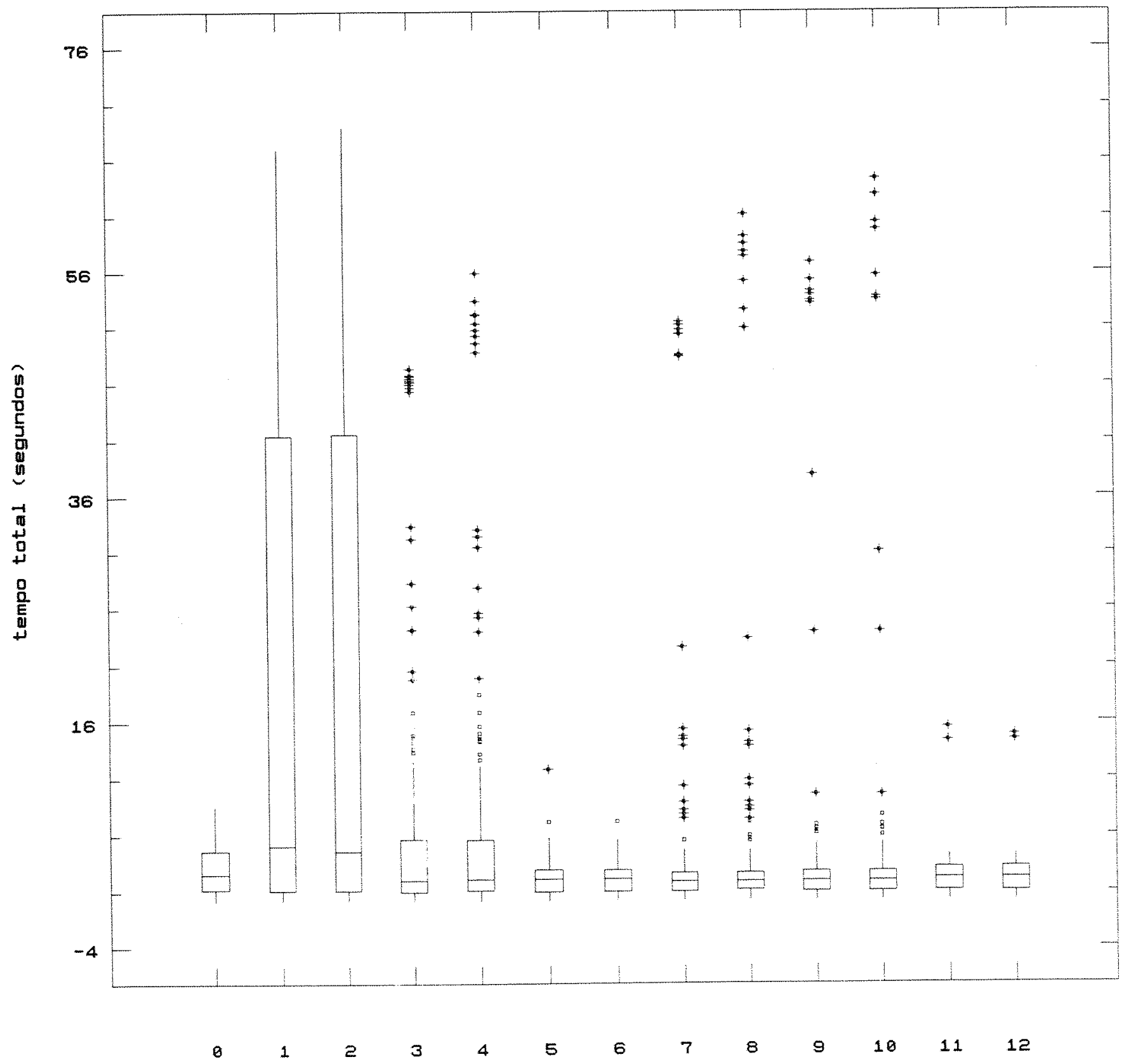

uariacoes 
Eliminacao $3 \quad(n=10)$

Distribuicao de tempo por suas variacoes

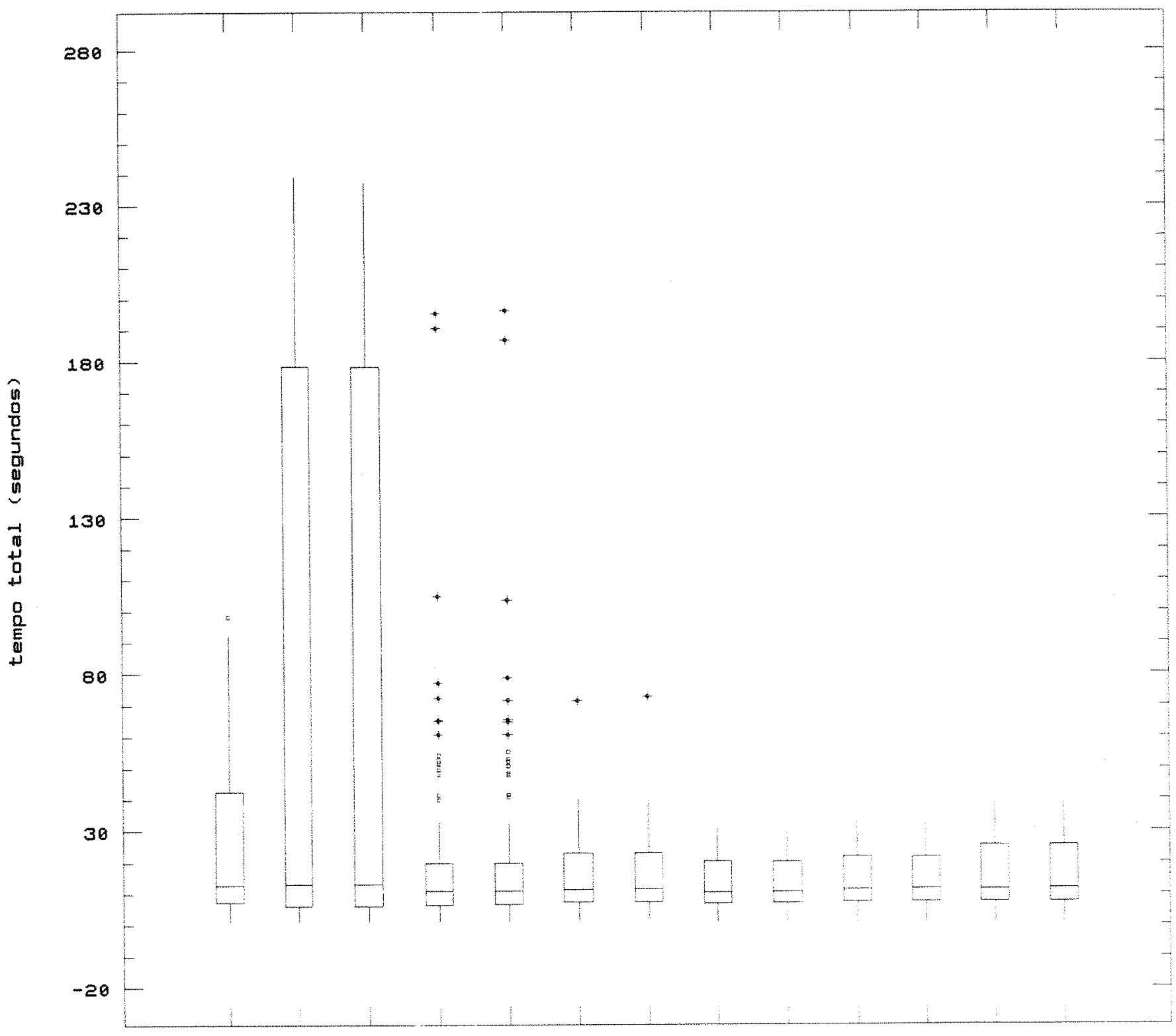

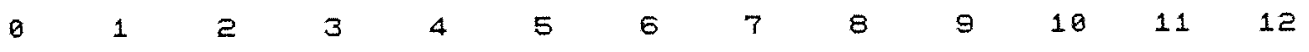


Eliminacao $3(n=20)$

Distribuicao de tempo por wus uariacoes

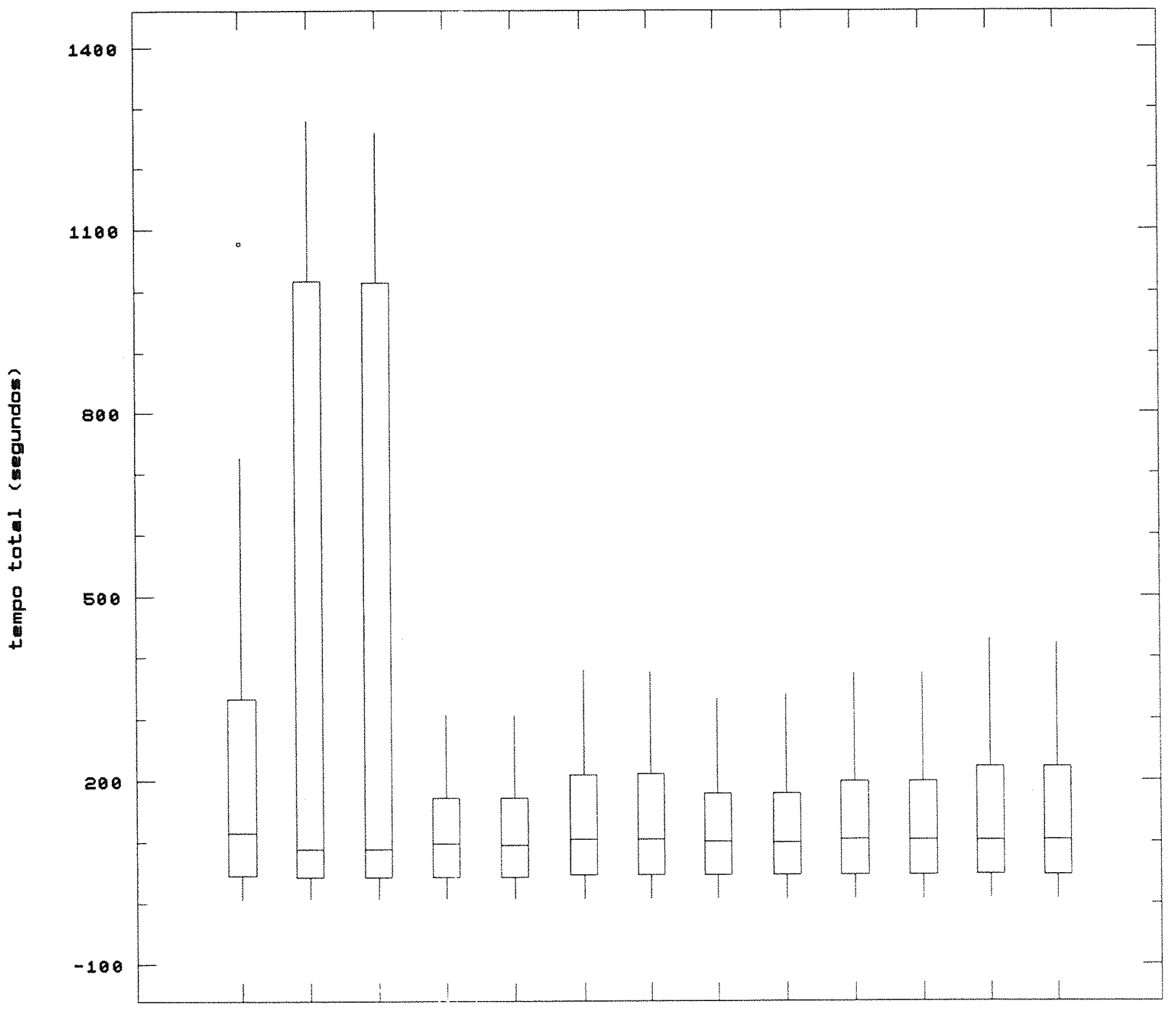

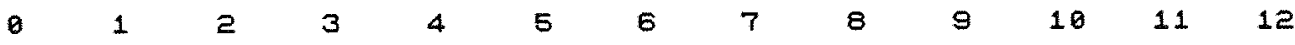




\subsubsection{Estratégia de eliminação 4}

A diferença desta estratégia para a eliminação 2 é que permitimos mais eliminações toda vez que há um decréscimo suficiente de $\left(u^{k}-l_{-}^{k}\right)$. Observando as tabelas de economia, vemos que mantendo $2 n$ cortes obtivemos resultados comparáveis aos da eliminação 2 . Destacamos as variações 1 e 2 para $n=5$, que pioraram muito o tempo em relação ao algoritmo sem eliminação.

Comparando com a eliminação 3 , que dá preferência às últimas restrições, vemos que obtivemos melhores resultados que naquele caso, mostrando que dar preferência a restrições associadas a pontos com valores menores de função objetivo é aconselhável para este tipo de problema.

$$
n=5
$$

\begin{tabular}{|c|c|c|c|c|c|c|c|c|c|}
\hline \multirow{3}{*}{ passo } & tam. & \multicolumn{6}{|c|}{ Quantidade de restriçōes mantidas } \\
\cline { 3 - 9 } & do & \multicolumn{3}{|c|}{$\mathrm{n}+1$} & \multicolumn{3}{|c|}{$2 \mathrm{n}$} \\
\cline { 3 - 9 } & sep & tempo & iter. & Total Hess & $\#$ & tempo & iter. & Total Hess \\
\hline $\mathrm{n}$ & $\mathrm{d}$ & 1 & $-219 \%$ & $-421 \%$ & $-442 \%$ & 7 & $31 \%$ & $-5 \%$ & $-3 \%$ \\
$\mathrm{n}$ & $\mathrm{d} / \mathrm{n}$ & 2 & $-224 \%$ & $-421 \%$ & $-443 \%$ & 8 & $31 \%$ & $-5 \%$ & $-3 \%$ \\
$2 \mathrm{n}$ & $\mathrm{d}$ & 3 & $11 \%$ & $-42 \%$ & $-41 \%$ & 9 & $\star 36 \%$ & $4 \%$ & $8 \%$ \\
$2 \mathrm{n}$ & $\mathrm{d} / \mathrm{n}$ & 4 & $2 \%$ & $-52 \%$ & $-53 \%$ & 10 & $\star 36 \%$ & $4 \%$ & $8 \%$ \\
$4 \mathrm{n}$ & $\mathrm{d}$ & 5 & $\star 34 \%$ & $2 \%$ & $5 \%$ & 11 & $30 \%$ & $3 \%$ & $6 \%$ \\
$4 \mathrm{n}$ & $\mathrm{d} / \mathrm{n}$ & 6 & $\star 34 \%$ & $2 \%$ & $5 \%$ & 12 & $29 \%$ & $3 \%$ & $6 \%$ \\
\hline
\end{tabular}

$$
n=10
$$

\begin{tabular}{|c|c|c|c|c|c|c|c|c|c|}
\hline \multirow{3}{*}{ passo } & tam. & \multicolumn{6}{|c|}{ Quantidade de restriçôes mantidas } \\
\cline { 3 - 9 } & do & \multicolumn{6}{|c|}{ n+1 } & \multicolumn{4}{|c|}{$2 \mathrm{n}$} \\
\cline { 3 - 9 } & sep. & $\#$ & tempo & iter. & Total Hess & $\#$ & tempo & iter. & Total Hess \\
\hline $\mathrm{n}$ & $\mathrm{d}$ & 1 & $35 \%$ & $-29 \%$ & $-24 \%$ & 7 & $\star 48 \%$ & $8 \%$ & $13 \%$ \\
$\mathrm{n}$ & $\mathrm{d} / \mathrm{n}$ & 2 & $35 \%$ & $-29 \%$ & $-24 \%$ & 8 & $\star 48 \%$ & $8 \%$ & $13 \%$ \\
$2 \mathrm{n}$ & $\mathrm{d}$ & 3 & $\star 51 \%$ & $6 \%$ & $12 \%$ & 9 & $43 \%$ & $7 \%$ & $11 \%$ \\
$2 \mathrm{n}$ & $\mathrm{d} / \mathrm{n}$ & 4 & $\star 50 \%$ & $6 \%$ & $11 \%$ & 10 & $43 \%$ & $7 \%$ & $11 \%$ \\
$4 \mathrm{n}$ & $\mathrm{d}$ & 5 & $43 \%$ & $5 \%$ & $9 \%$ & 11 & $37 \%$ & $5 \%$ & $9 \%$ \\
$4 \mathrm{n}$ & $\mathrm{d} / \mathrm{n}$ & 6 & $42 \%$ & $5 \%$ & $9 \%$ & 12 & $37 \%$ & $5 \%$ & $9 \%$ \\
\hline
\end{tabular}




$$
n=20
$$

\begin{tabular}{|c|c|c|c|c|c|c|c|c|c|}
\hline \multirow{3}{*}{ passo } & tam. & \multicolumn{6}{|c|}{ Quantidade de restrições mantidas } \\
\cline { 3 - 9 } & do & \multicolumn{3}{|c|}{$\mathrm{n}+1$} & \multicolumn{3}{|c|}{$2 \mathrm{n}$} \\
\cline { 3 - 9 } & sep. & $\#$ & tempo & iter. & Total Hess & $\#$ & tempo & iter. & Total Hess \\
\hline $\mathrm{n}$ & $\mathrm{d}$ & 1 & $41 \%$ & $-9 \%$ & $-3 \%$ & 7 & $\star 43 \%$ & $8 \%$ & $12 \%$ \\
$\mathrm{n}$ & $\mathrm{d} / \mathrm{n}$ & 2 & $41 \%$ & $-9 \%$ & $-3 \%$ & 8 & $\star 43 \%$ & $8 \%$ & $12 \%$ \\
$2 \mathrm{n}$ & $\mathrm{d}$ & 3 & $\star 45 \%$ & $5 \%$ & $10 \%$ & 9 & $37 \%$ & $6 \%$ & $10 \%$ \\
$2 \mathrm{n}$ & $\mathrm{d} / \mathrm{n}$ & 4 & $\star 45 \%$ & $5 \%$ & $10 \%$ & 10 & $37 \%$ & $6 \%$ & $10 \%$ \\
$4 \mathrm{n}$ & $\mathrm{d}$ & 5 & $37 \%$ & $4 \%$ & $8 \%$ & 11 & $31 \%$ & $4 \%$ & $7 \%$ \\
$4 \mathrm{n}$ & $\mathrm{d} / \mathrm{n}$ & 6 & $37 \%$ & $4 \%$ & $8 \%$ & 12 & $31 \%$ & $4 \%$ & $7 \%$ \\
\hline
\end{tabular}


Eliminacao $4 \quad(n=5)$

Distribuicad de tempo por suas variacoes

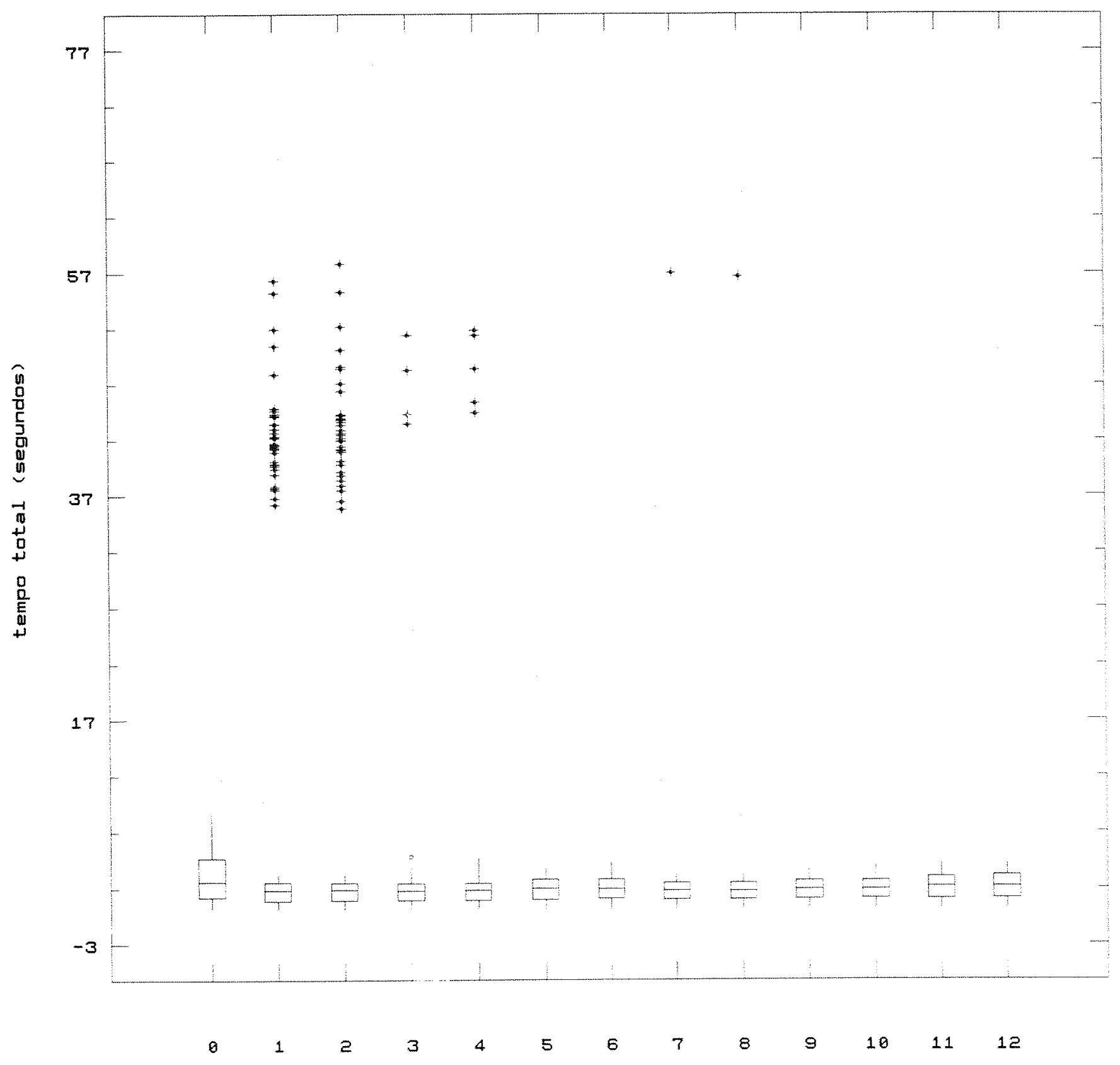

variacoes 
Eliminacer $4 \quad(n=10)$

Distribuicao de tempo por suas variacoes

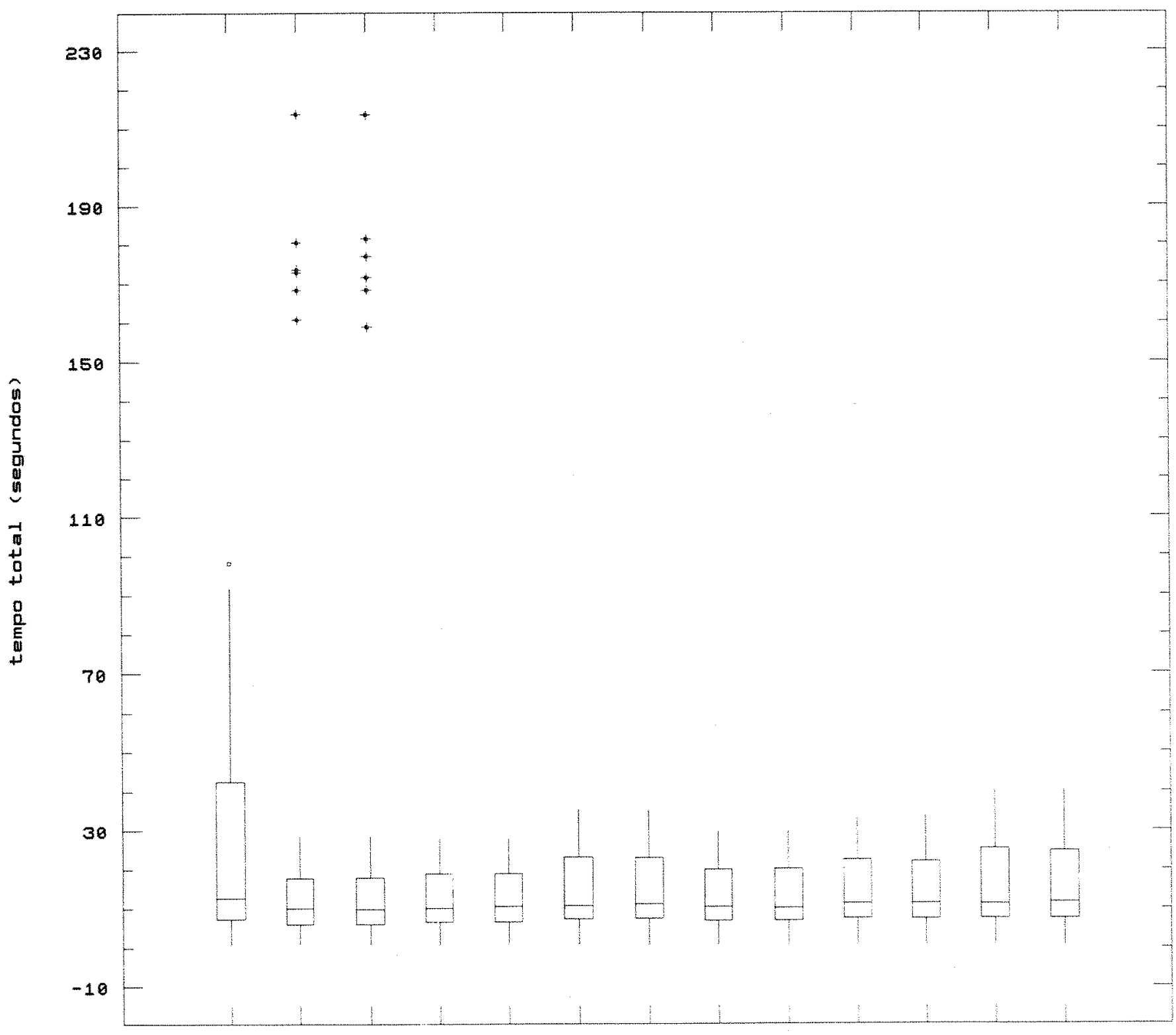

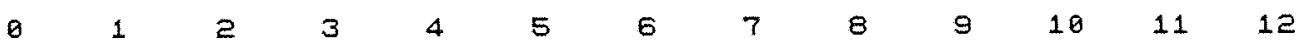

variacoes 
Eliminacao $4 \quad(n=20)$

Distribuicao de tempo por suas variacoes

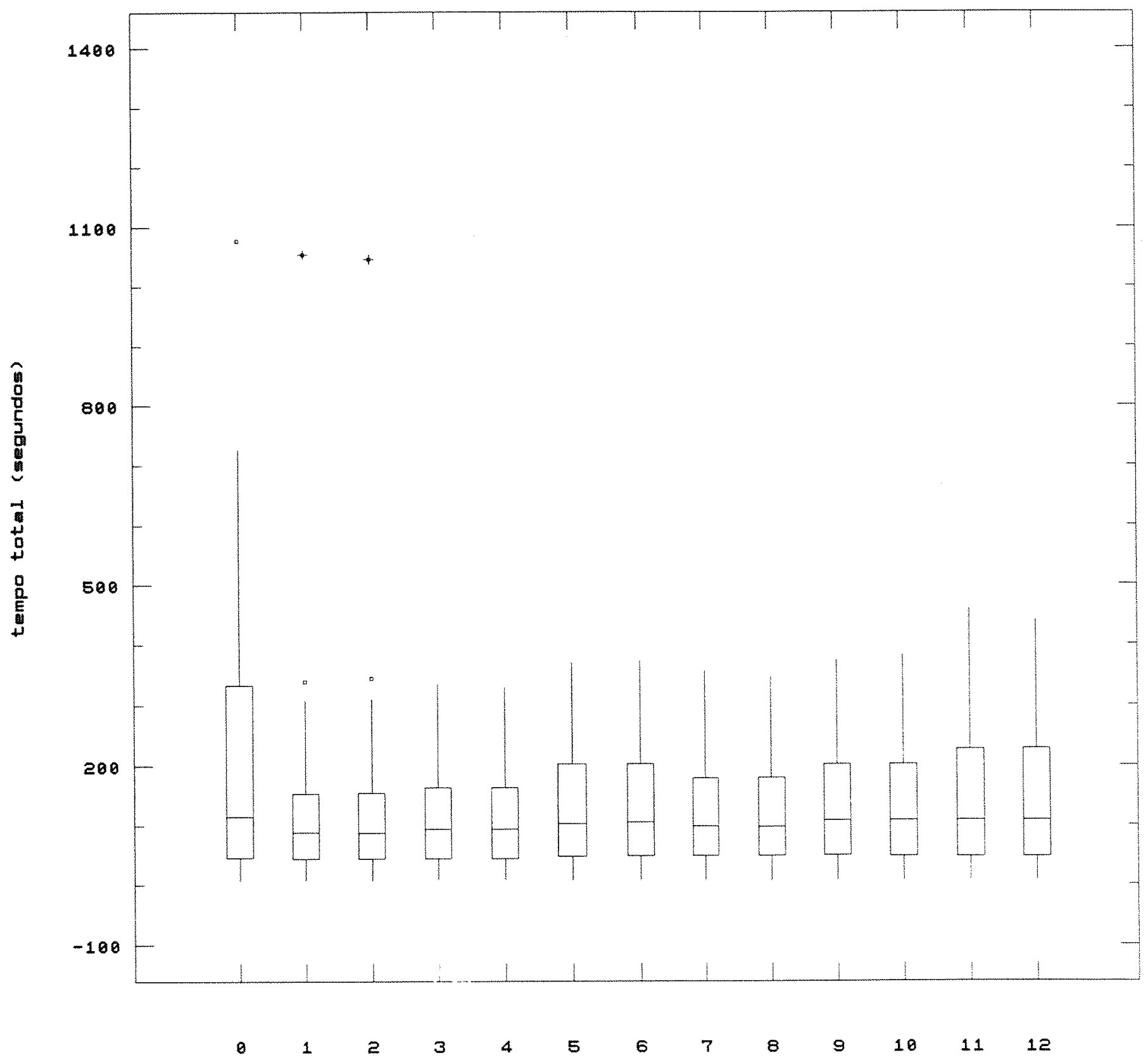

variacoes 


\subsubsection{Conclusões}

As estratégias de eliminação estudadas contribuíram com uma considerável economia de tempo para funções quadráticas sob restrições de box, sendo que as melhores variações das estratégias atingiram uma economia em torno de 40 a $50 \%$ em relação ao algoritmo sem eliminação.

O número de restrições mantidas e o tamanho do passo influenciaram a performance das estratégias, sendo que a influência não foi uniforme para as 4 estratégias.

Dar preferência a manter as restrições associadas a pontos de menor valor de função objetivo foi melhor que escolher as últimas restrições geradas. Acreditamos que esta conclusão pode ser bastante diferente para outros tipos de função .

Nem sempre eliminação de muitas restrições numa certa iteração trouxe vantagens nas iterações subseqüentes. Isto ocorreu porque eliminações de restrições em excesso em alguns casos aumentou muito o número de iterações e em outros dificultou eliminações futuras.

Em alguns casos de melhor economia de tempo, ocorreu que o número de restriçoes manteve-se praticamente constante ao longo das iterações. Isto sugere que pode ser que certas estratégias garantam a existência de um limite máximo de restrições por iteraçào. Este é um campo de investigação teórica que poderia ser explorado, e a comprovação desta conjectura garantiria que a complexidade por iteração dependeria apenas da dimensão do problema. 
Apêndice A

Gráficos sobre os parâmetros das quadráticas 
Distribuicao de tempo egundo noond

Algoritmo sem liminacone $n=5$

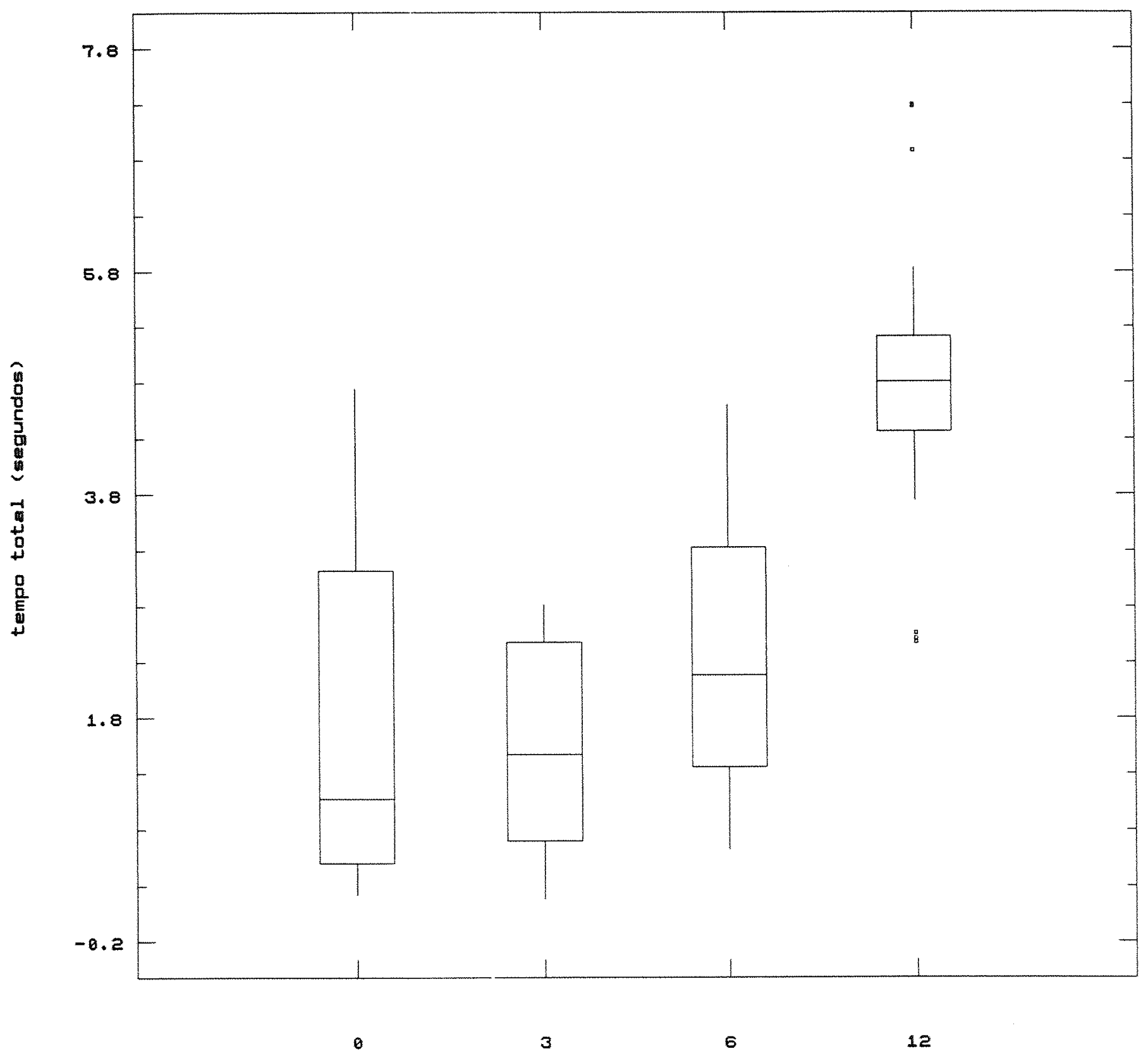

neond 
Distribuicao de \#teracom mequndo ncond

Algoritmo sem eliminaco $n=5$

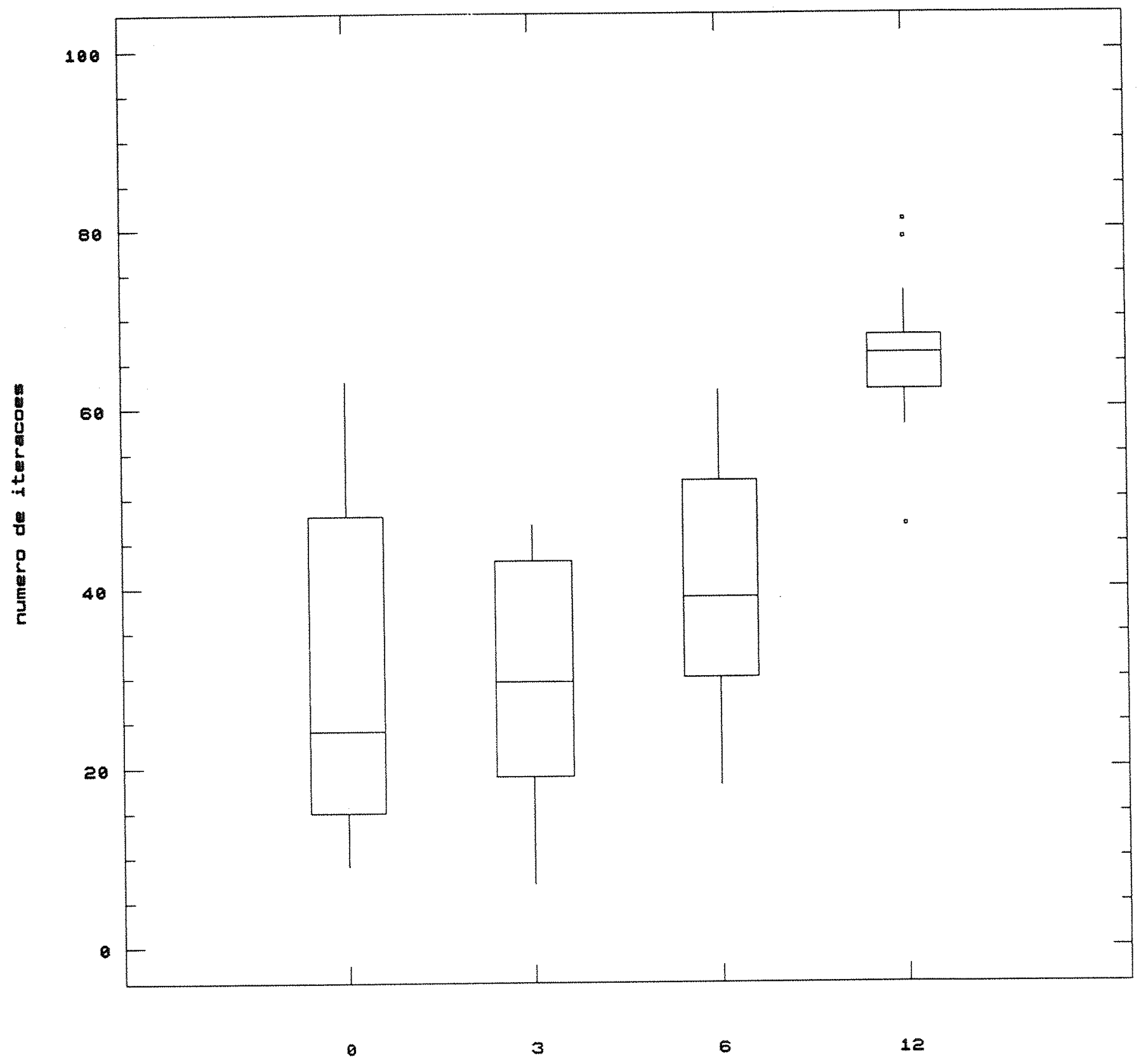

neond 
uistribuicao de tempo megunda neond

Algoritmo sem eliminacoes $n=20$

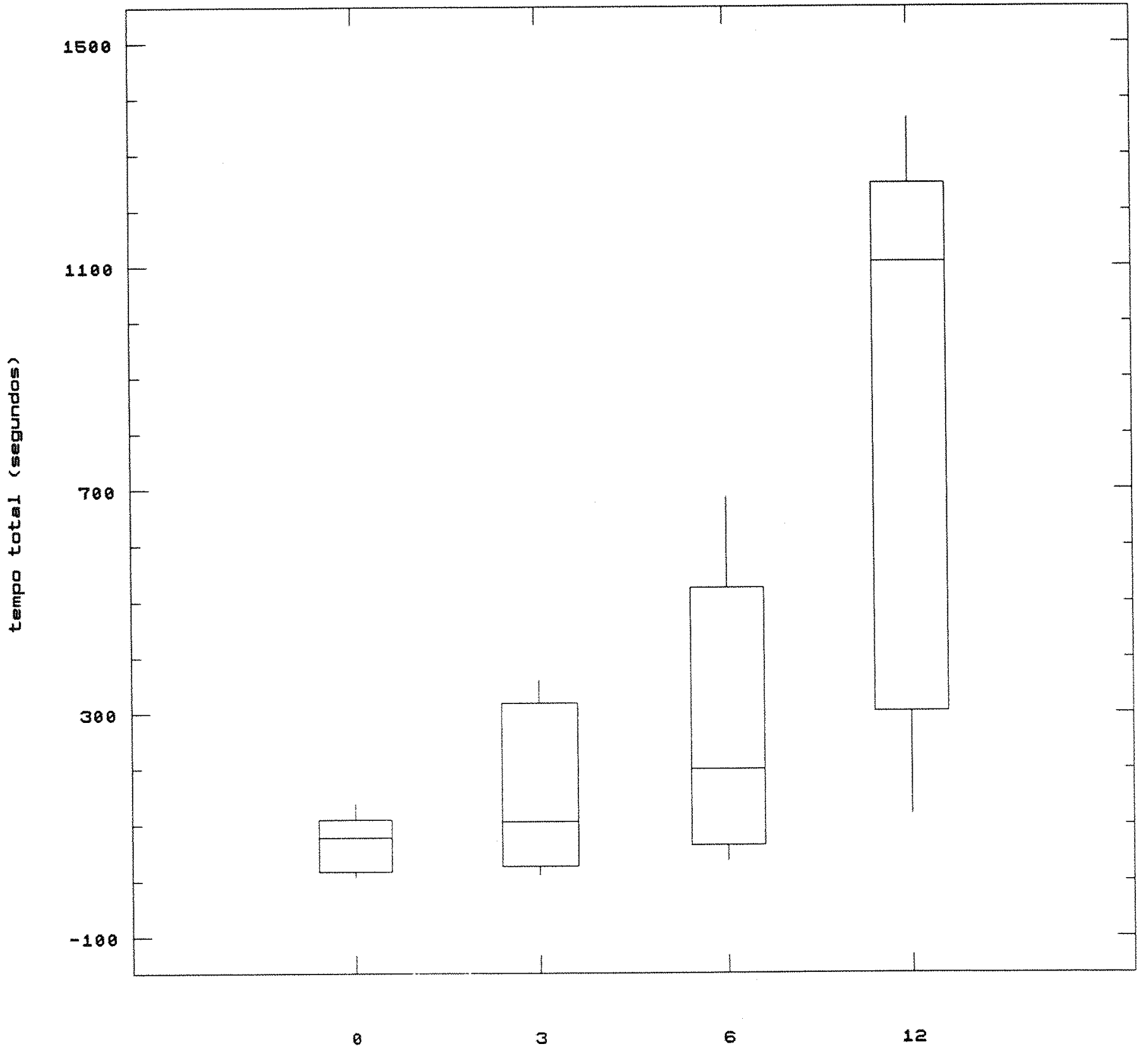

neond 
Distribuicao de \#iteracoes wegundo neond

Algoritmo Eem eliminacoes $n=26$

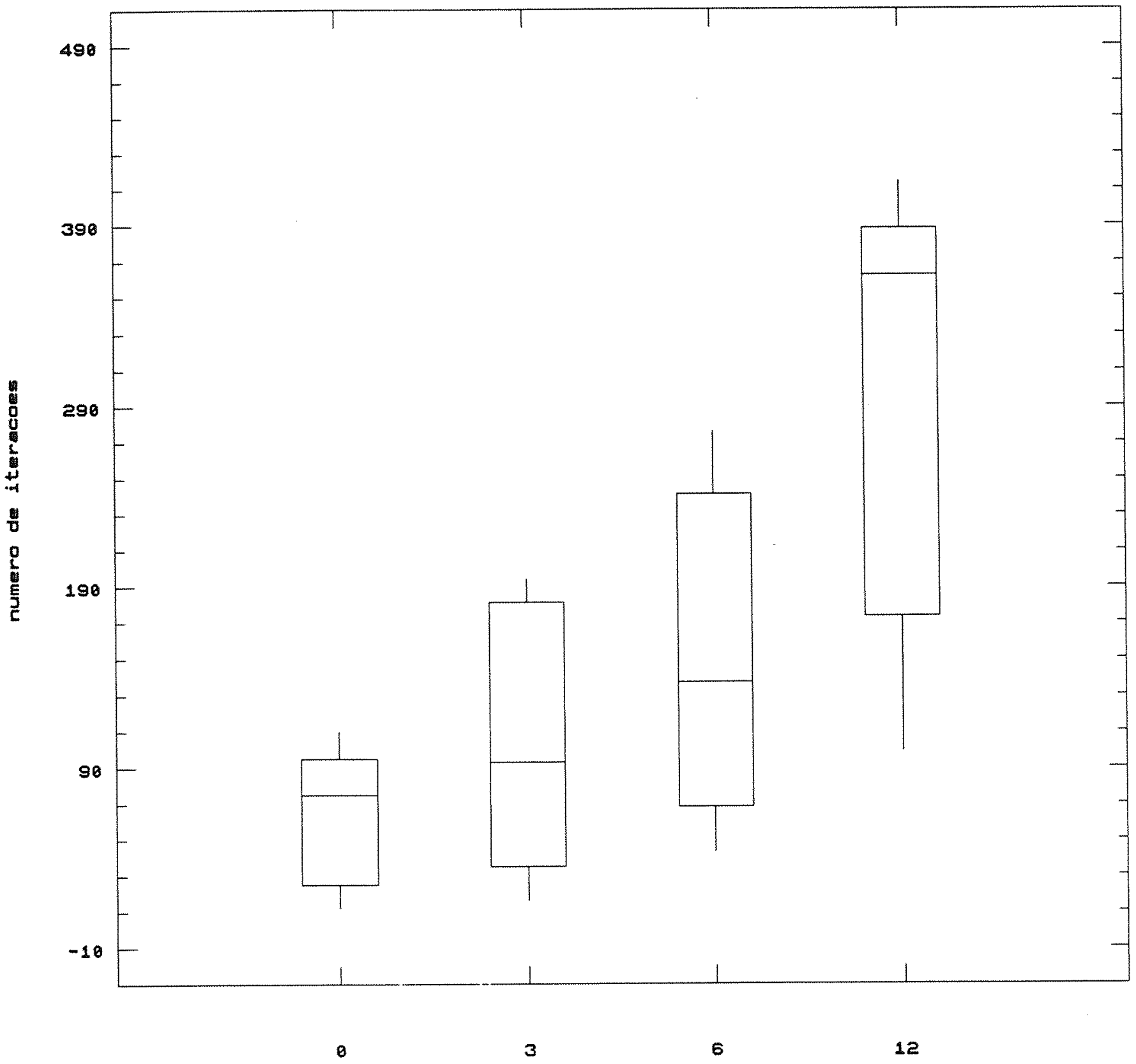

ncond 
Distribuicao de tempo segundo na $\left(x^{*}\right)$

Algoritmo sem eliminacoes $n=5$

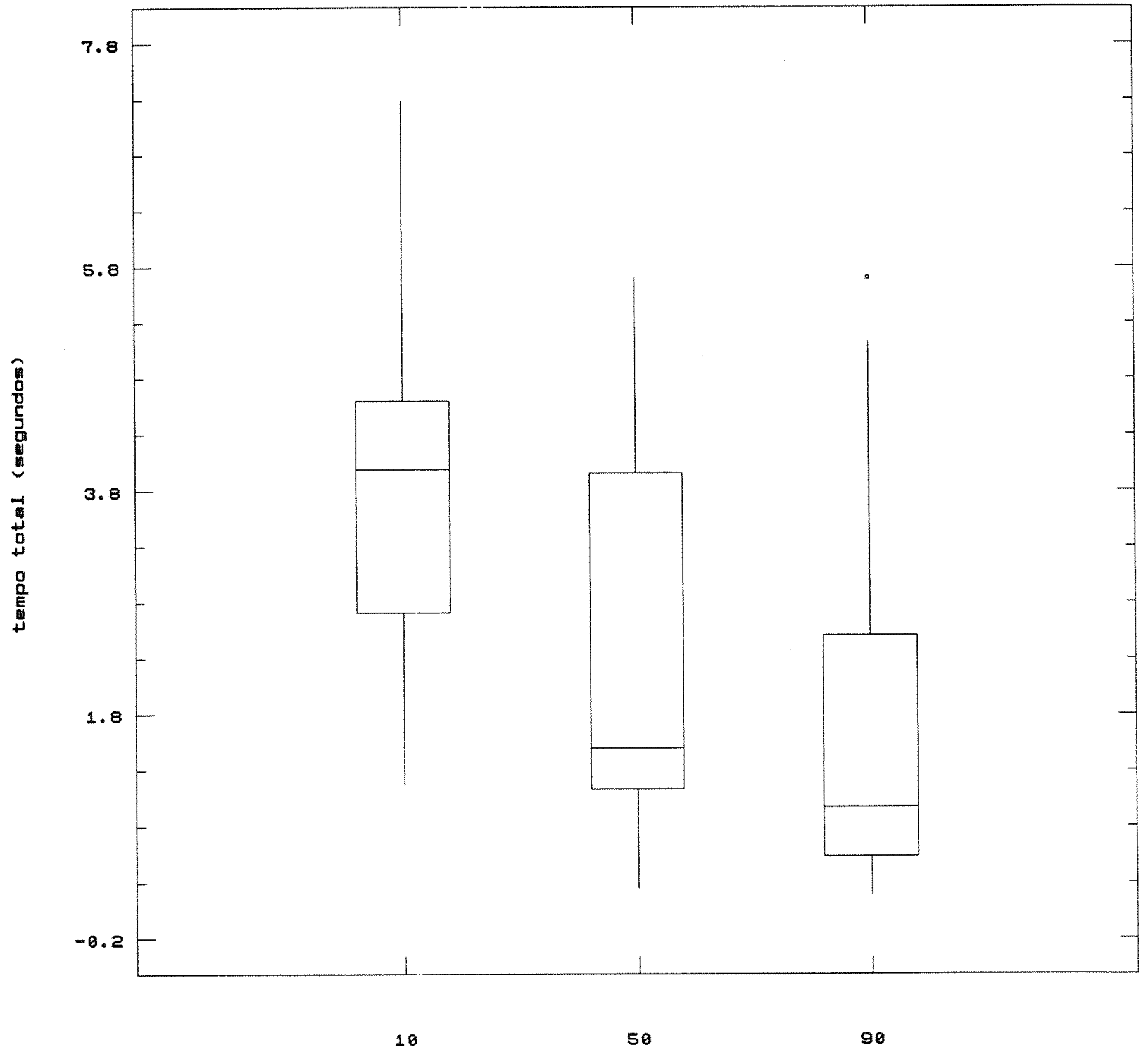

na $\left(x^{*}\right)$ (em \% do numero de restricoes) 
Distrib. de \#iteracoes segundo na $\left(x^{*}\right)$

Algoritmo sem eliminacoes $n=6$

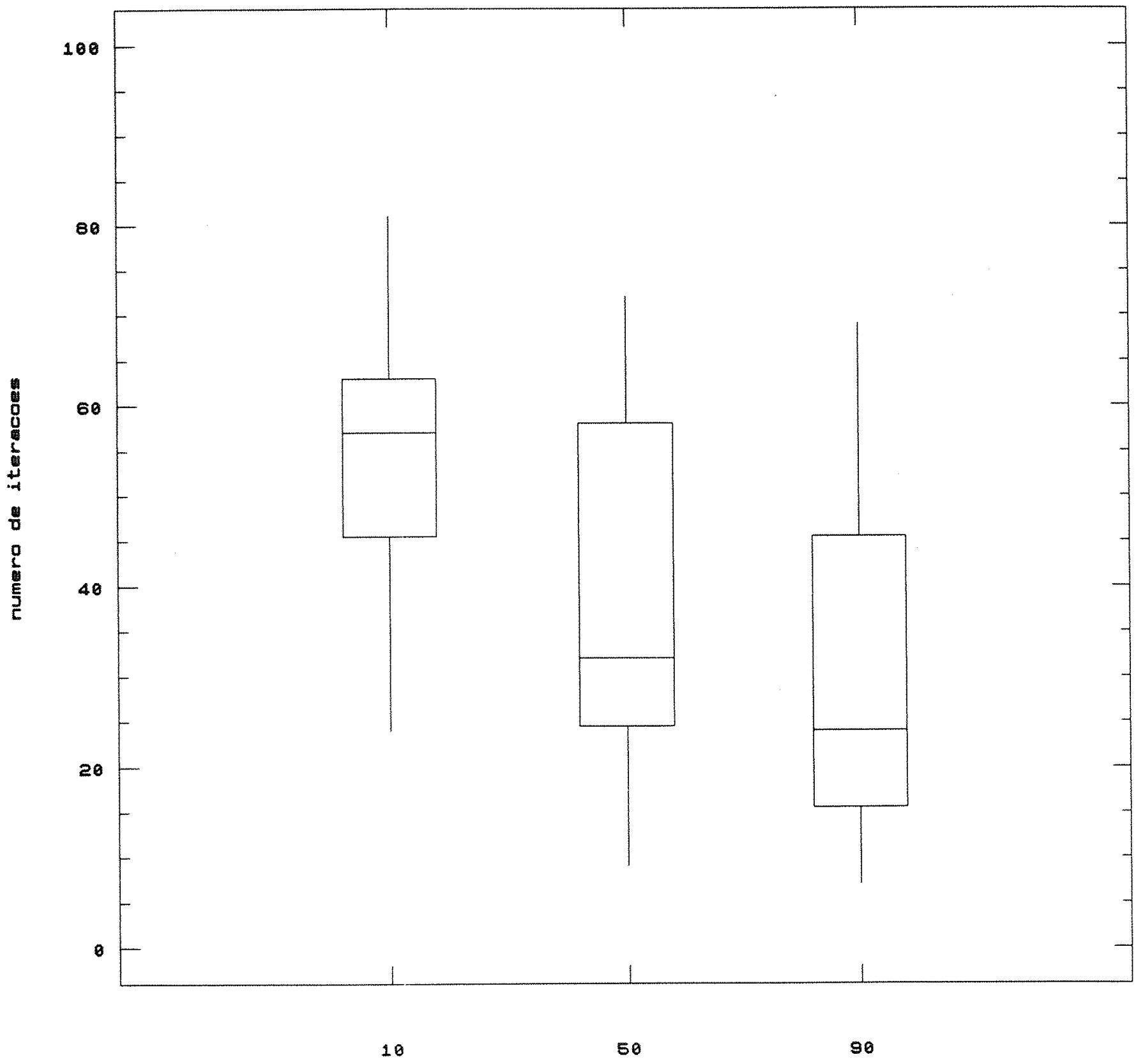

na $\left(x^{*}\right)$ (em \% do numero de restricoes) 
Distribuicao de tempo segundo na $\left(x^{*}\right)$

Algoritmo mem eliminacom $n=10$

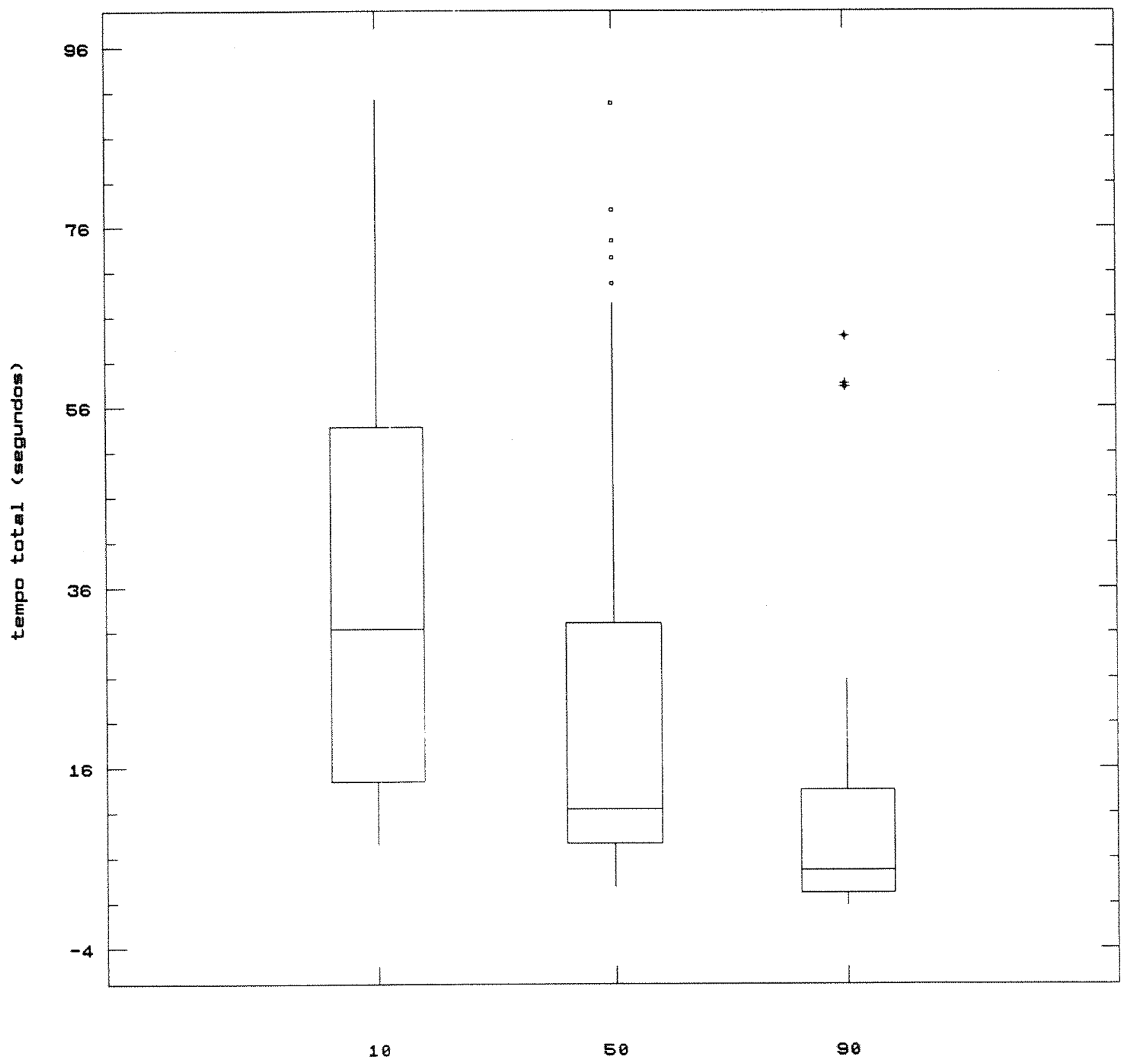

na $\left(x^{\star}\right)$ (em $\%$ do numero de restricoes) 
Distrib. de Ateracoes segundo $n$ (x*)

Algoritmo em eliminacoes $n=10$

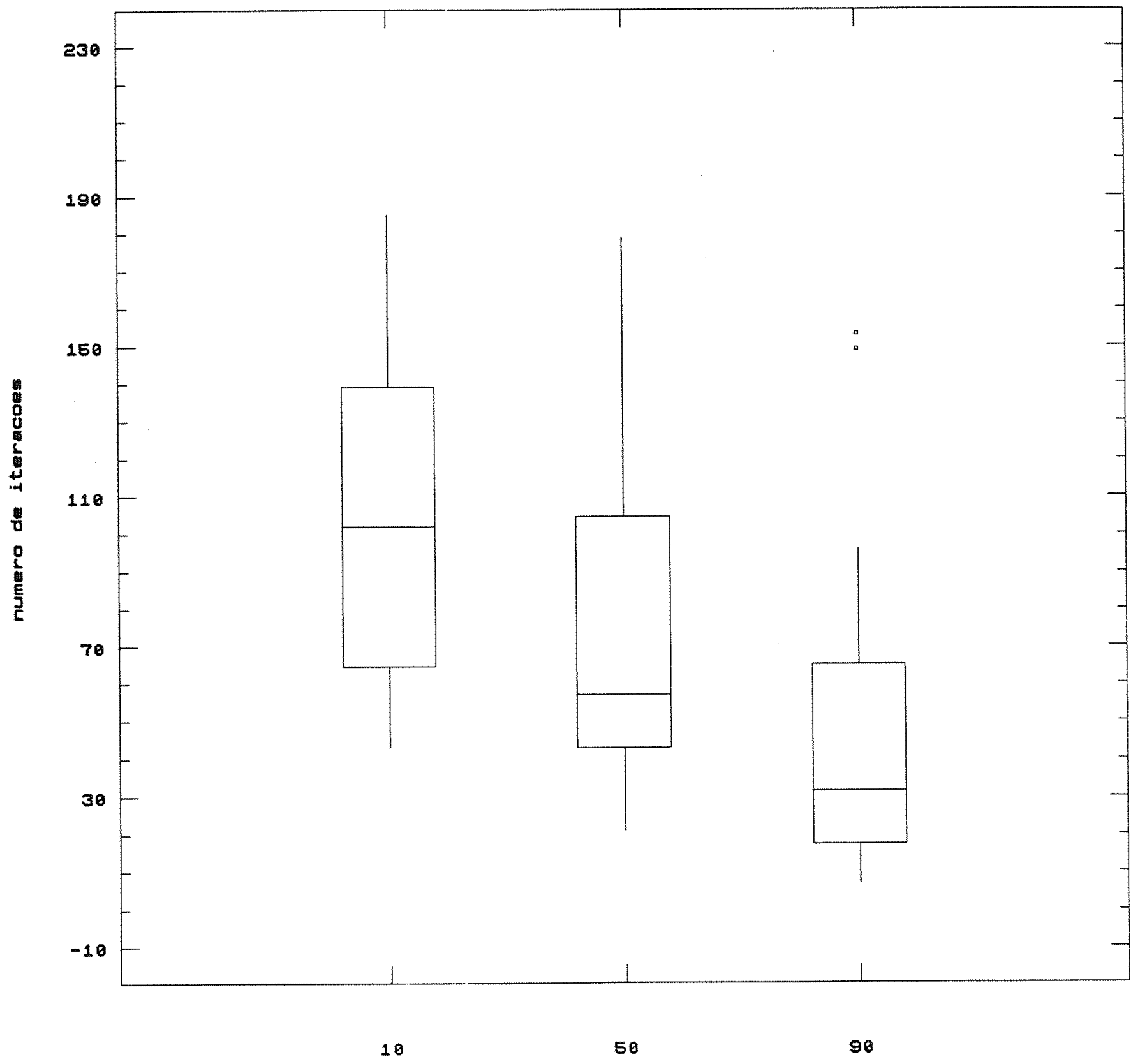

$n a\left(x^{*}\right)$ (em \% do numero de restricoes) 
Distribuicao de tempo megundo ndeg

Algoritmo sem eliminacoes $n=5$

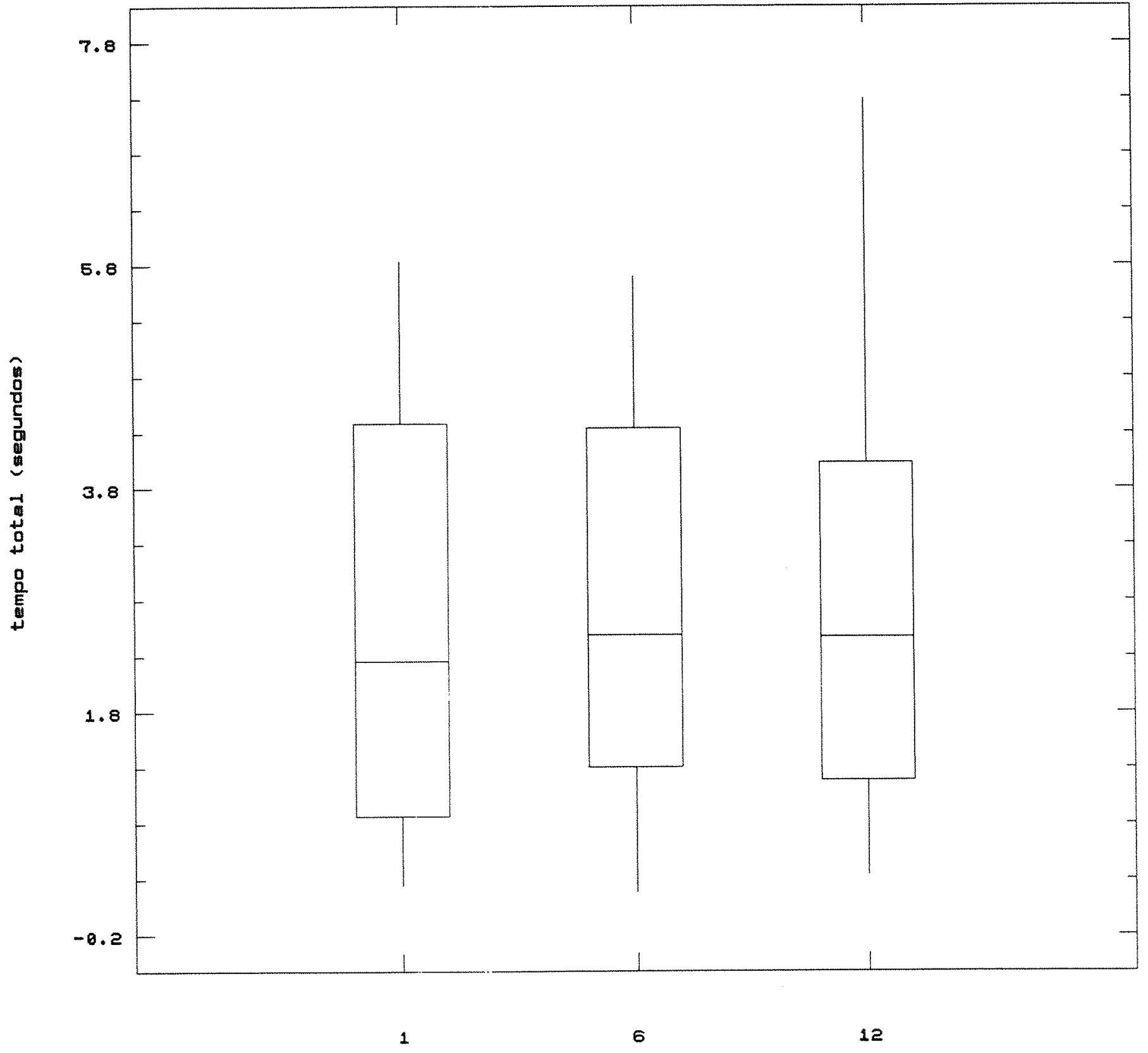

ndeg 
Distribuicao de iteracoes segundo ndeg

Algoritmo sem eliminacom $n=5$

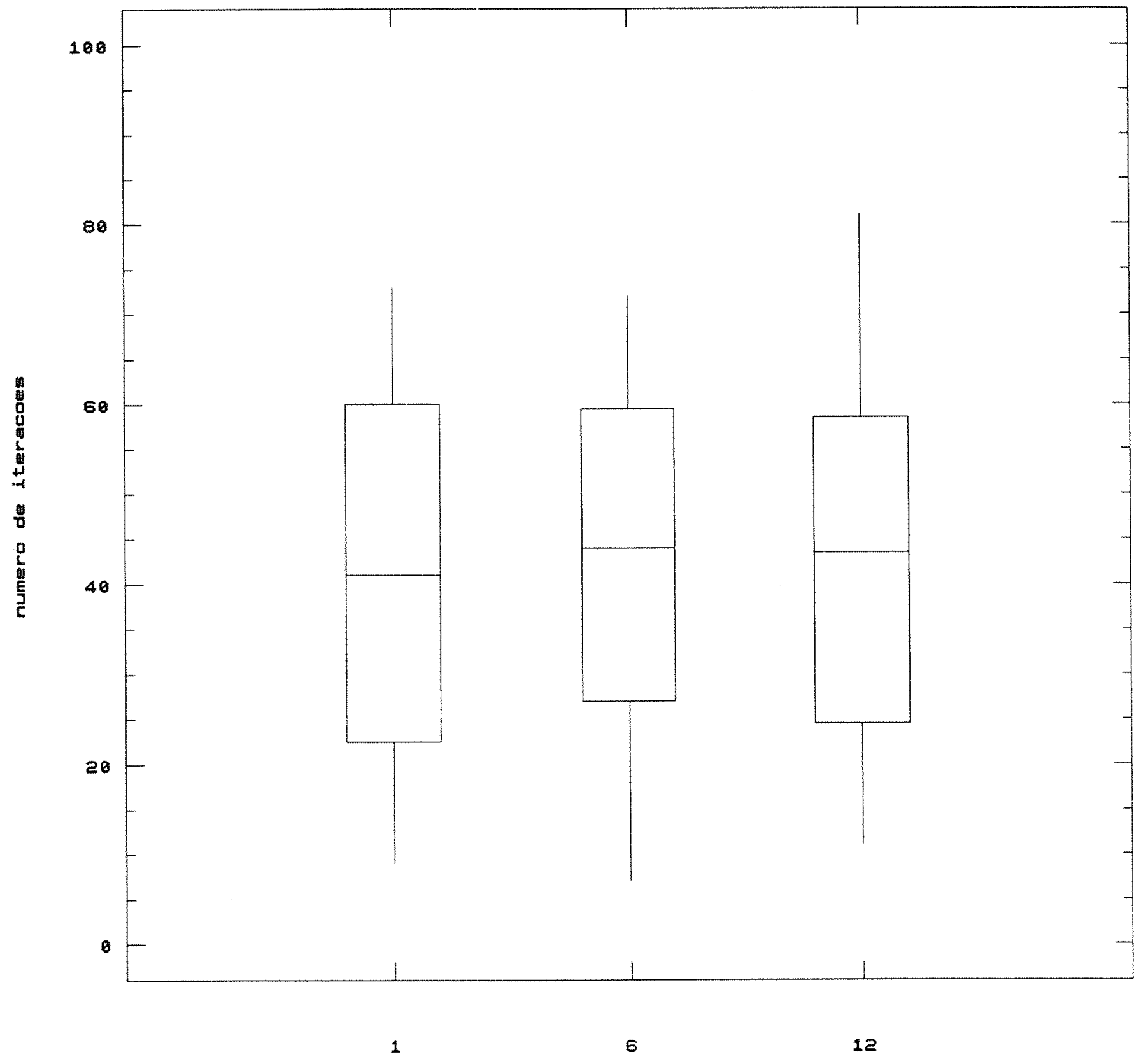

ndeg 
oistribuicao de tempo segundo ndeg

Algoritmo sem liminacoes $n=26$

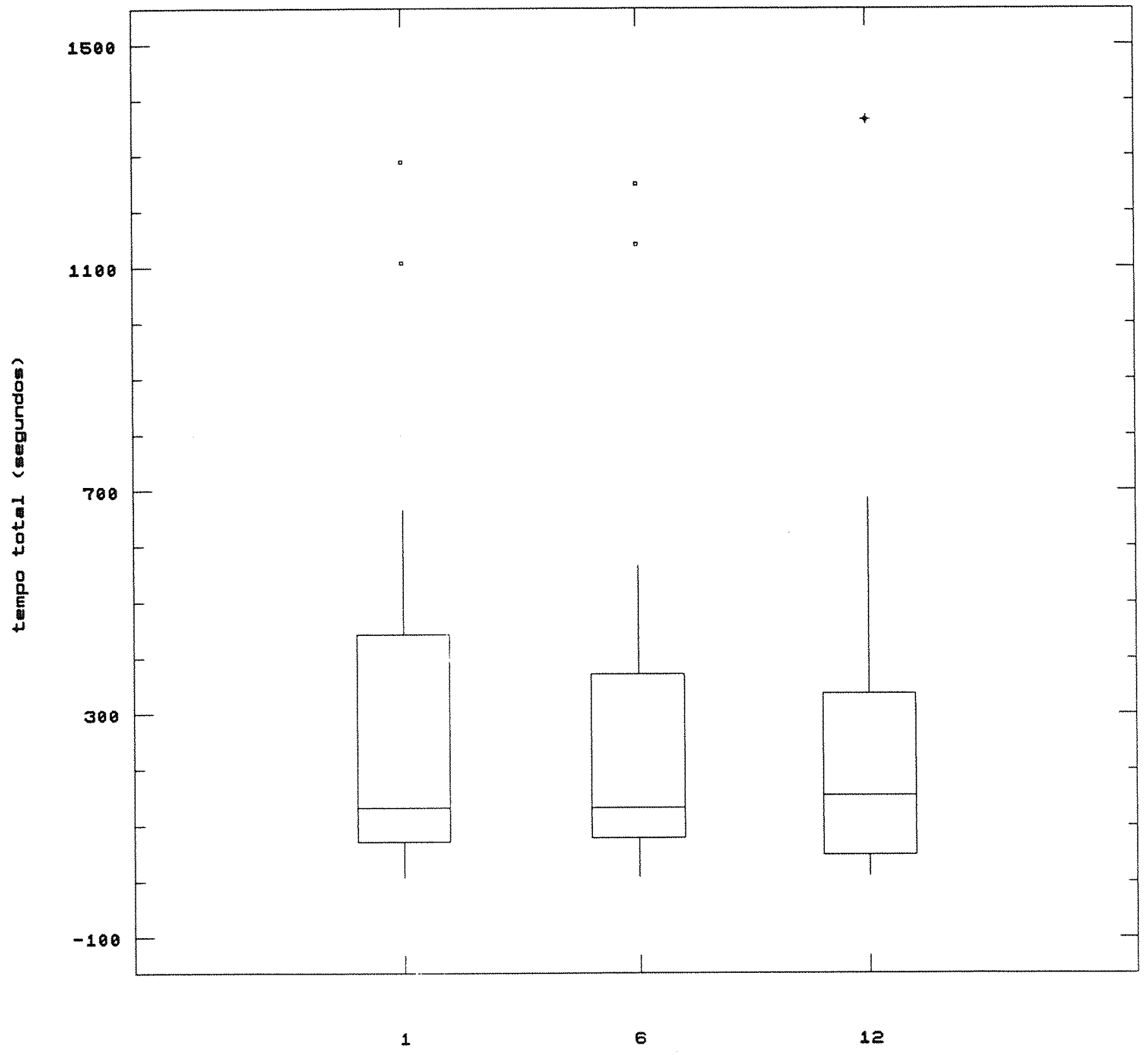

ndeg 
Distribuicao de \#iteracos gundo ndeg

Algoritmo oem eliminacoes $n=20$

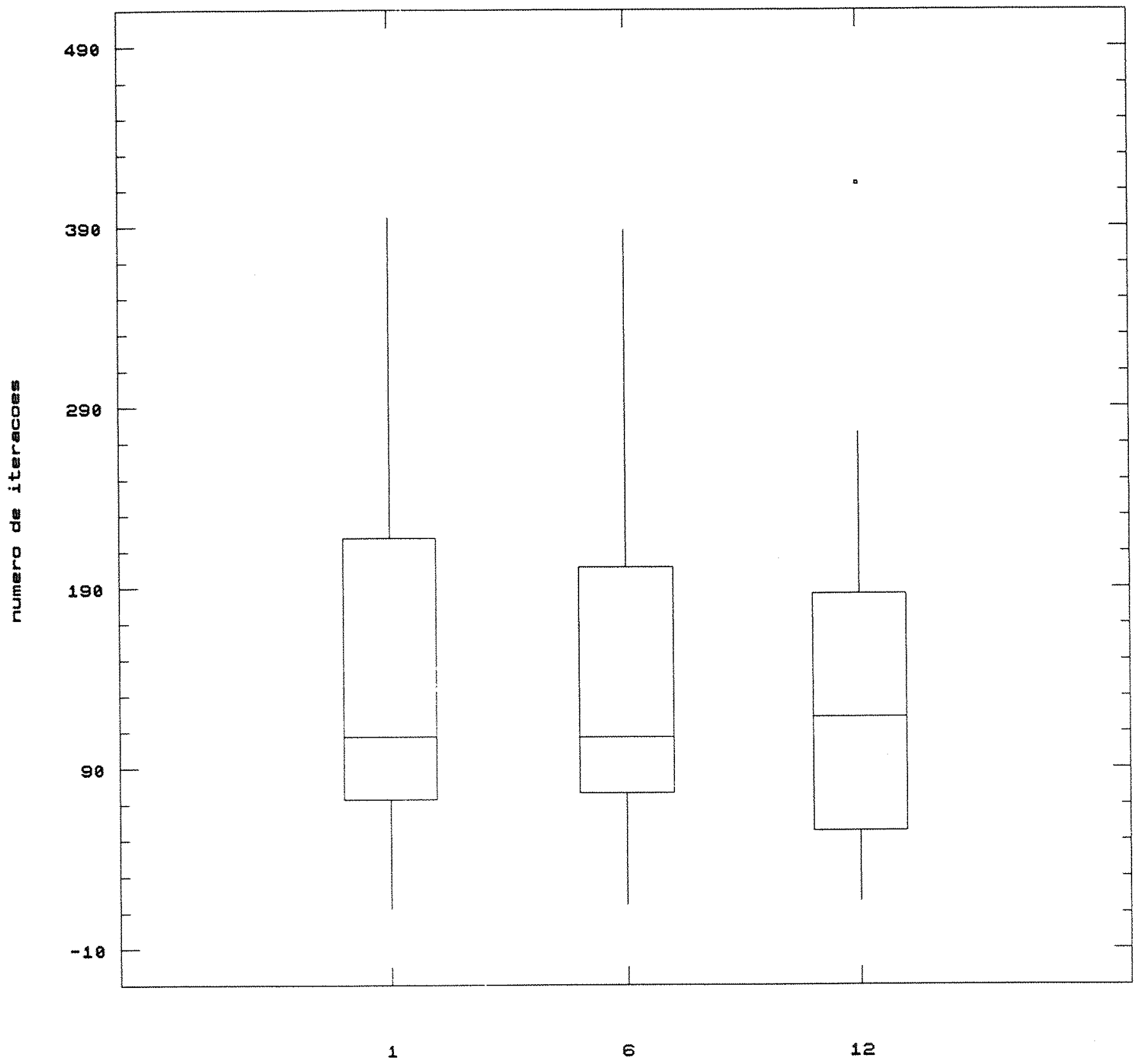

ndeg 
Dietribuicao de tempo equndo na $(x \theta)$

Algoritmo sem eliminacoes $n=5$

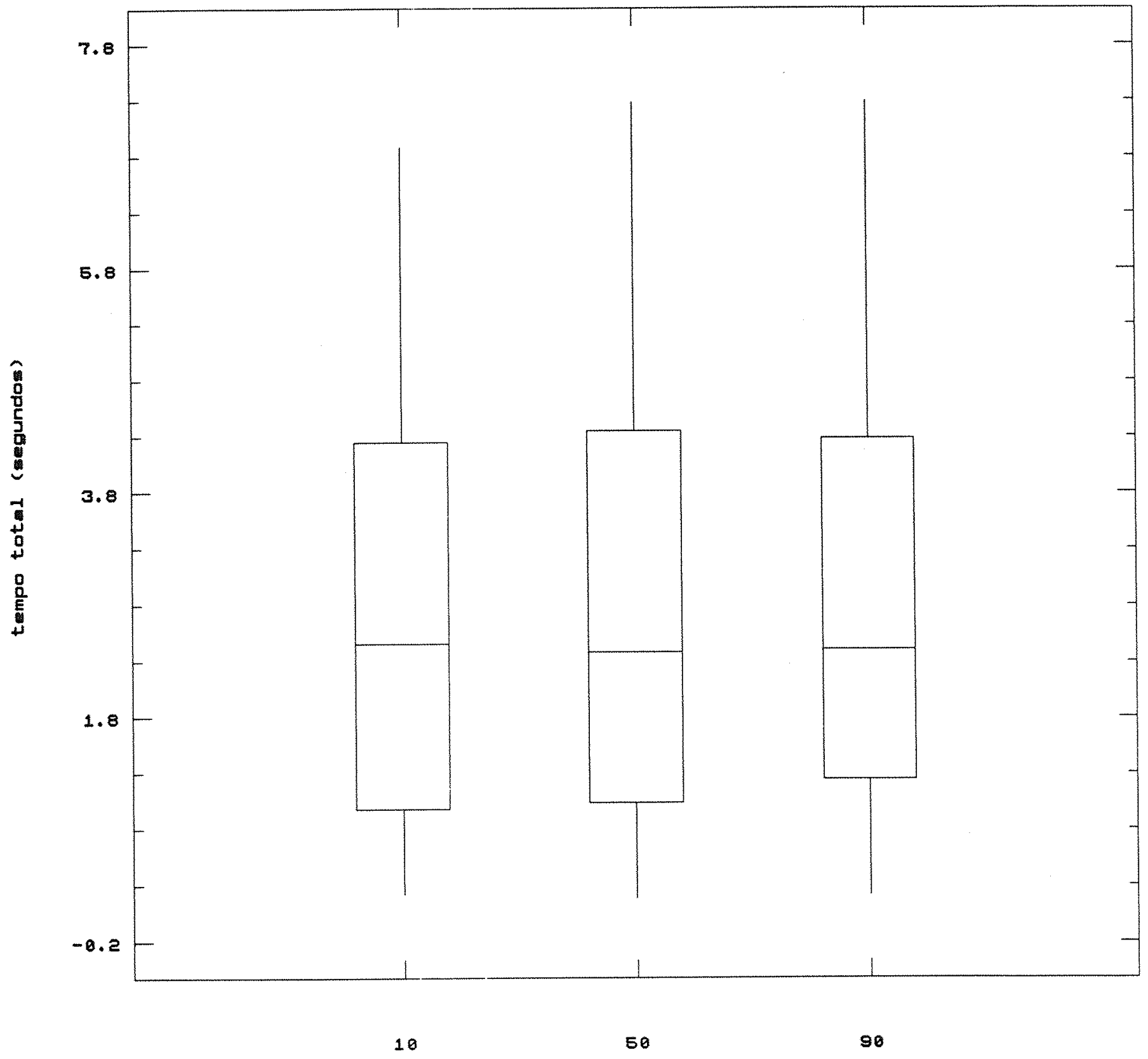

na $(x \theta)$ (em $\%$ do numero de restricoes) 
Distrib. de \#iteracoes segundo na $(x \theta)$

Algoritmo Em eliminacoes $n=5$

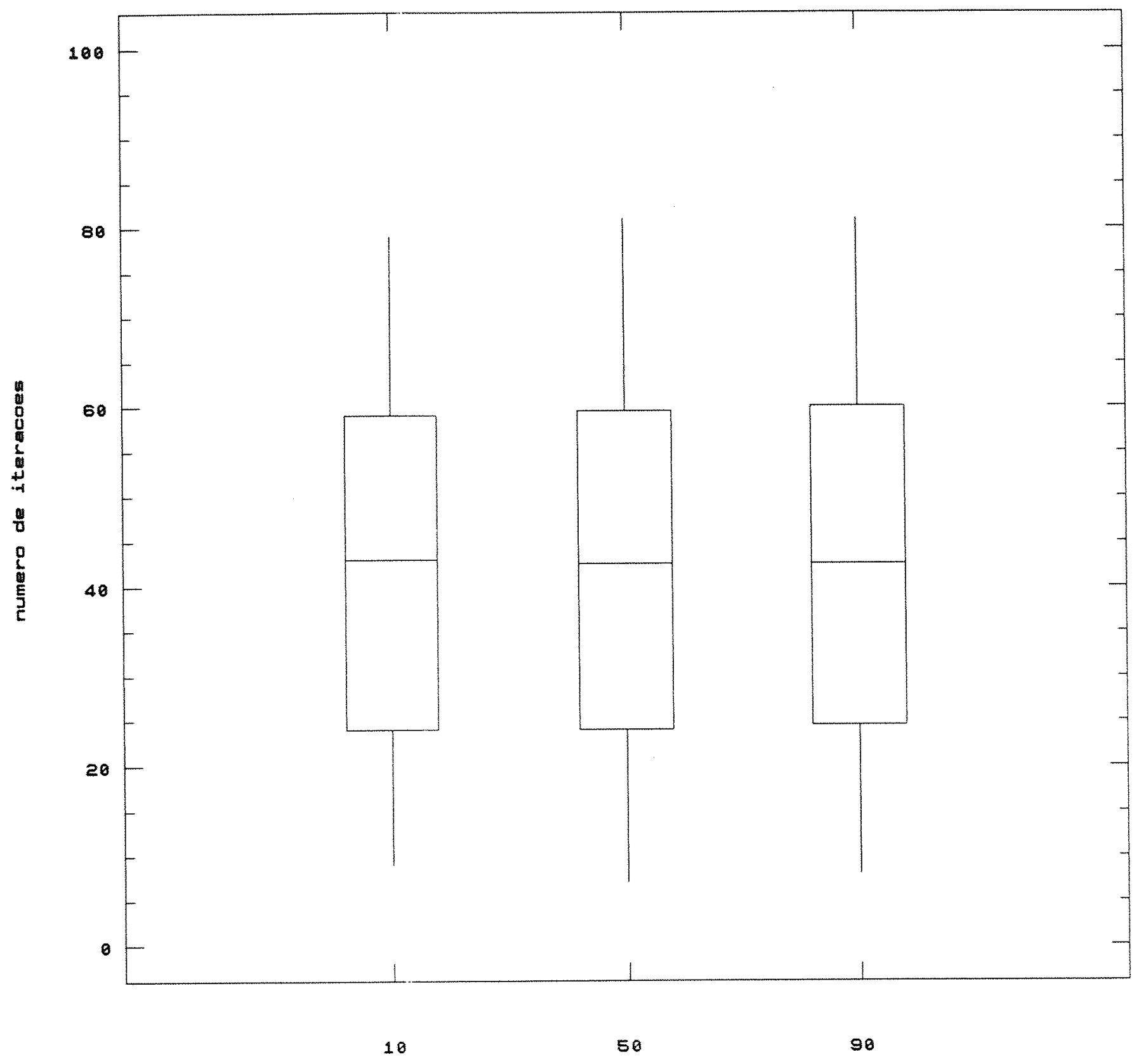

na( $x \theta$ ) (em \% do numero de restricoes) 


\section{Apêndice B}

Gráficos para eliminações 1 e 2 
Eliminacao $1 \quad(n=5)$

Distribuicao de tempo por suas variacoes

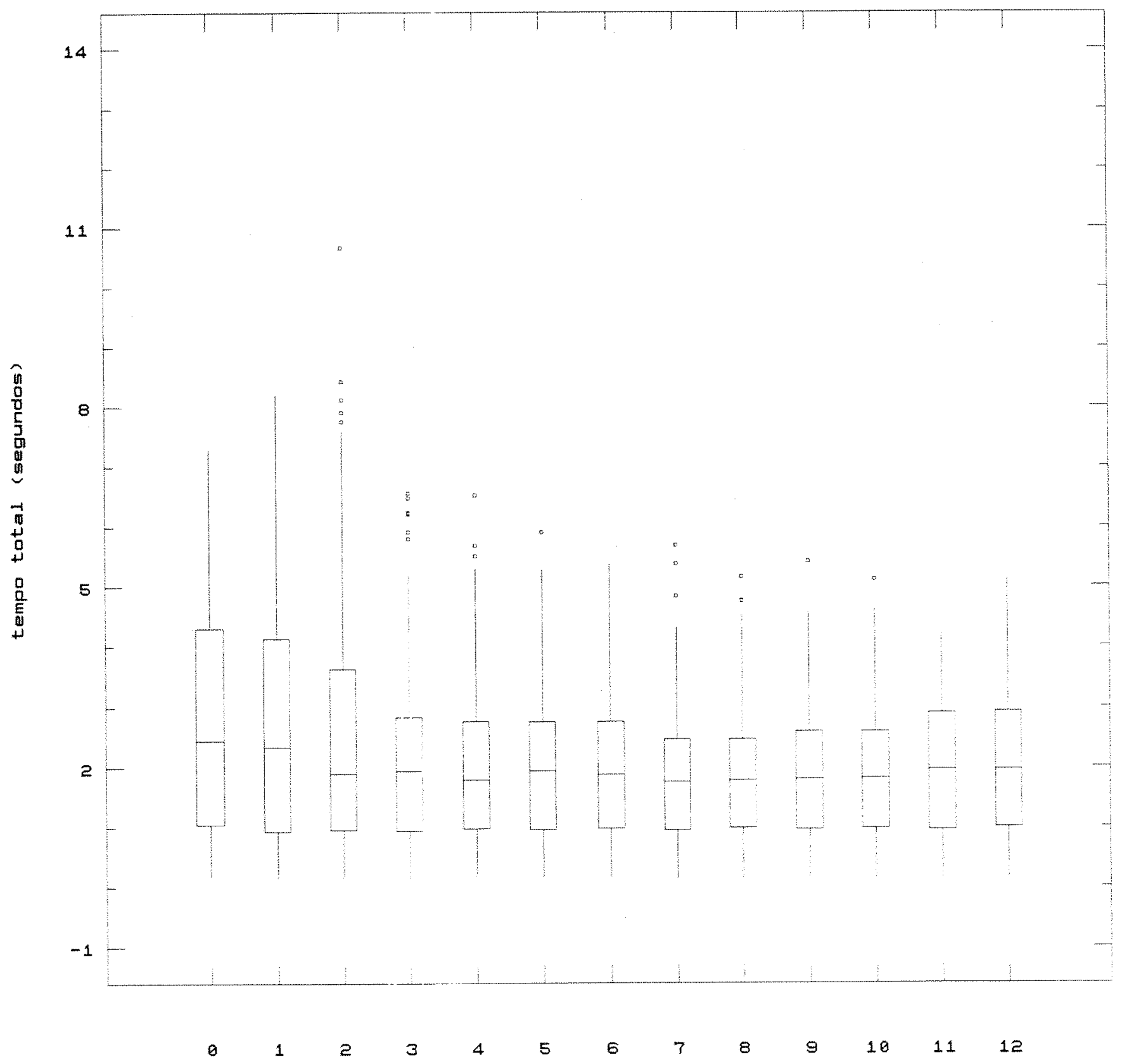

uariacoes 
Eliminacao $1(n=28)$

Distribuicao de tempo por suas variacoes

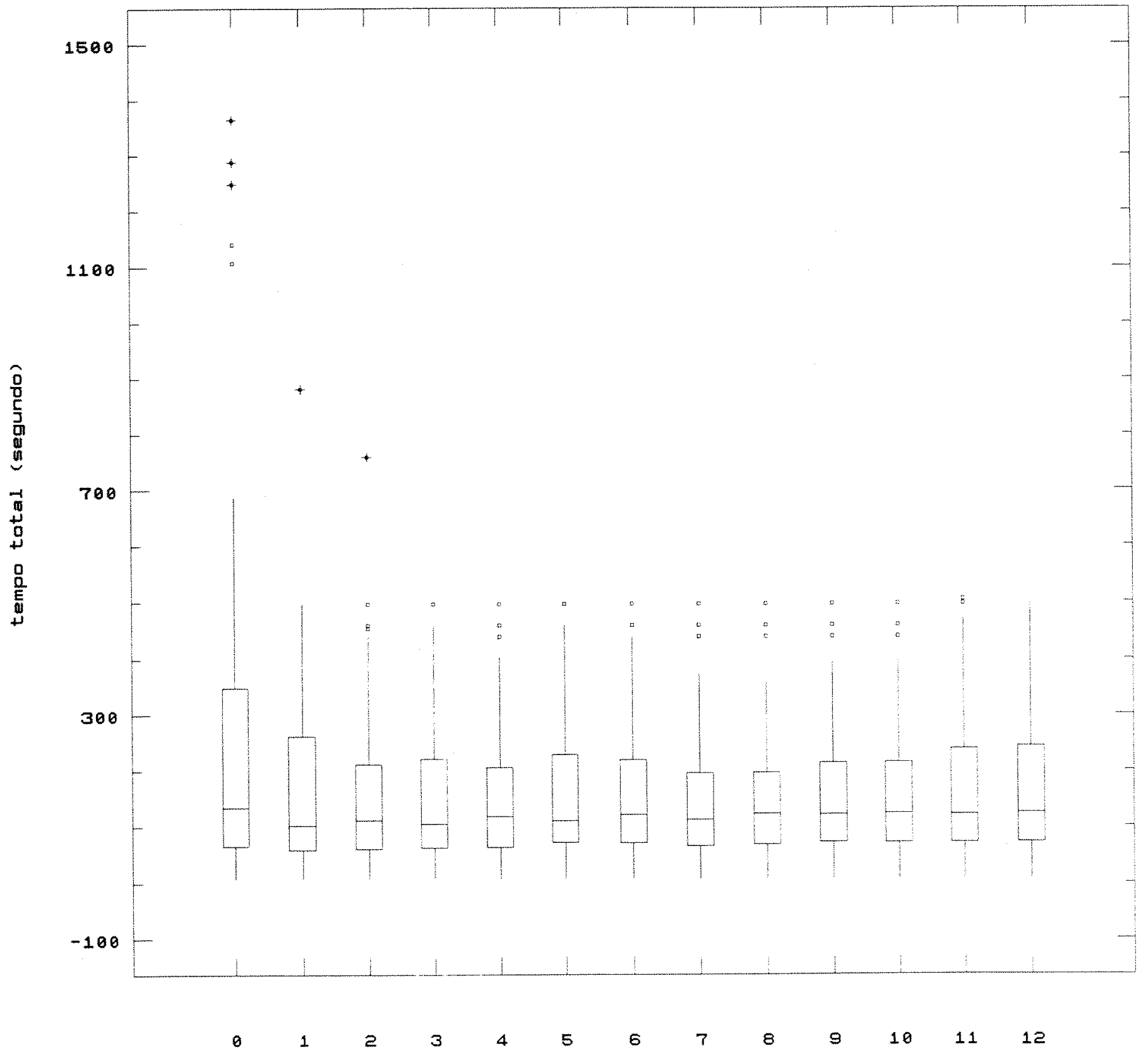


Eliminacao $1 \quad(n=5)$

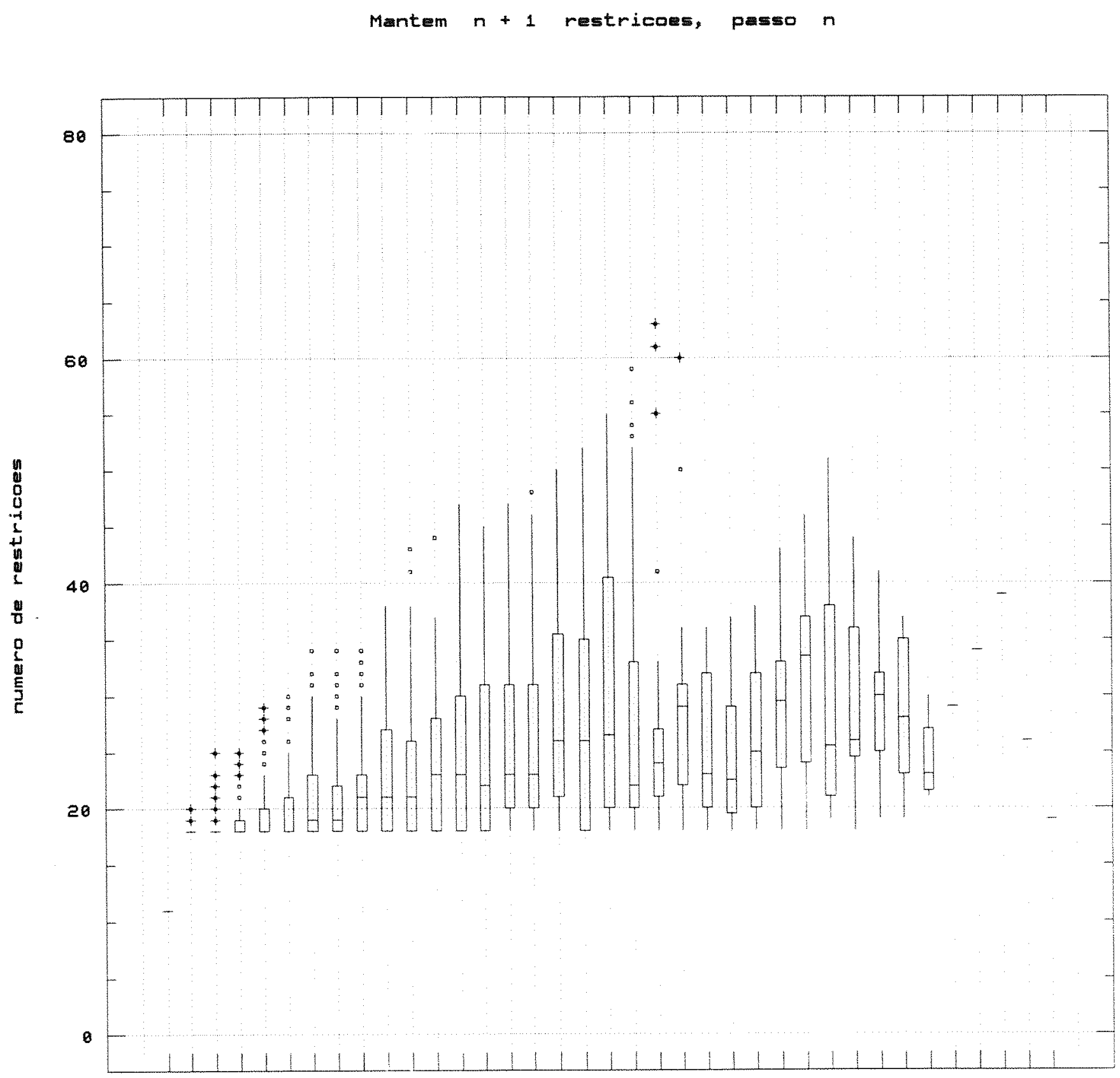

$\begin{array}{lllllllllllllllllllllll}0 & 15 & 25 & 35 & 45 & 55 & 65 & 75 & 85 & 95 & 105 & 115 & 125 & 135 & 145 & 155 & 165 & 175 & 185\end{array}$

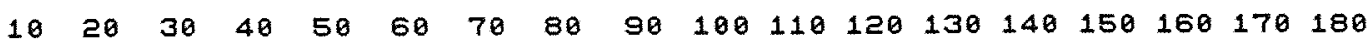


Eliminsedo $1 \quad(n=5)$

Mantm $2 n$ restricoss, passon $n$

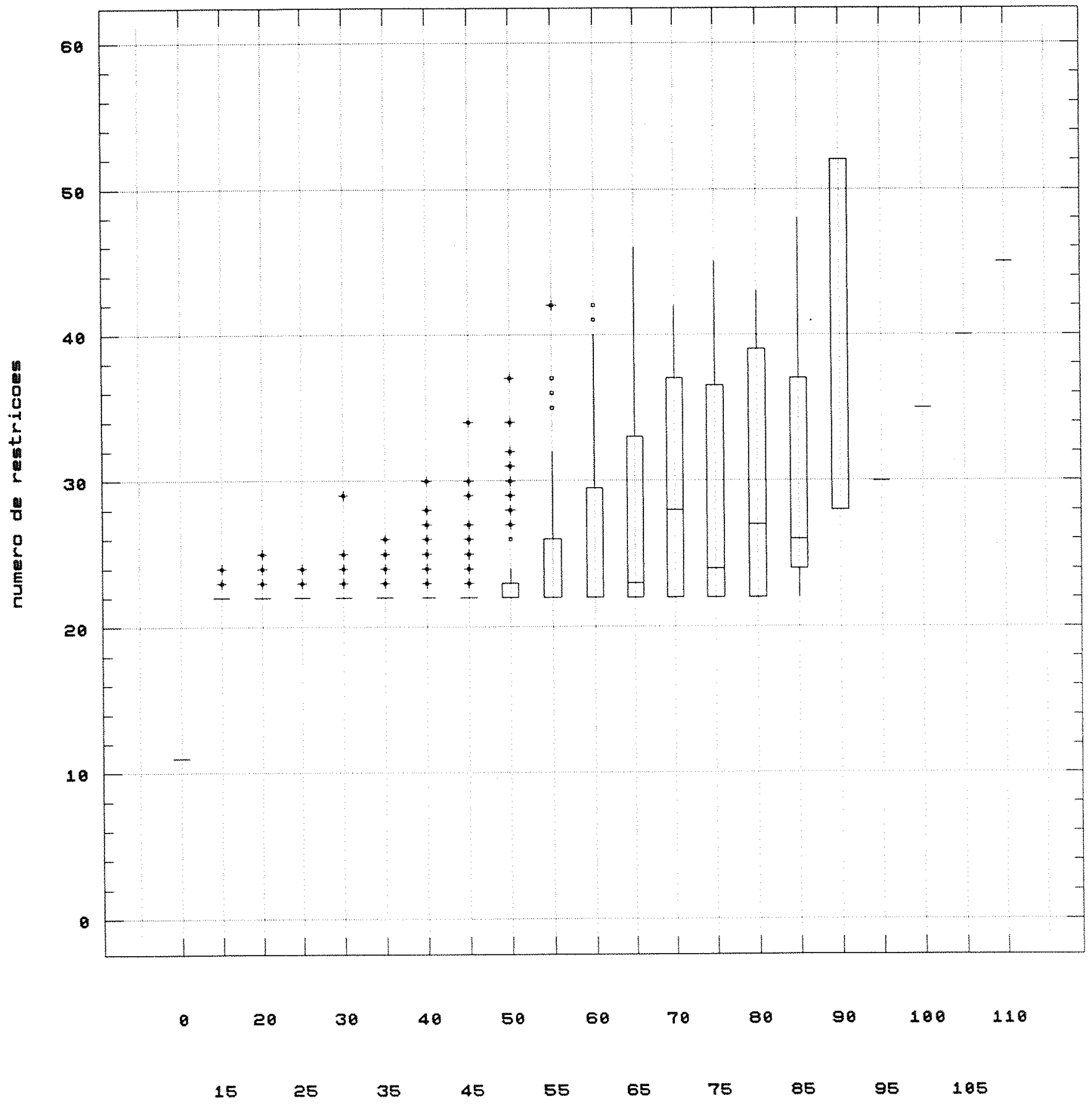




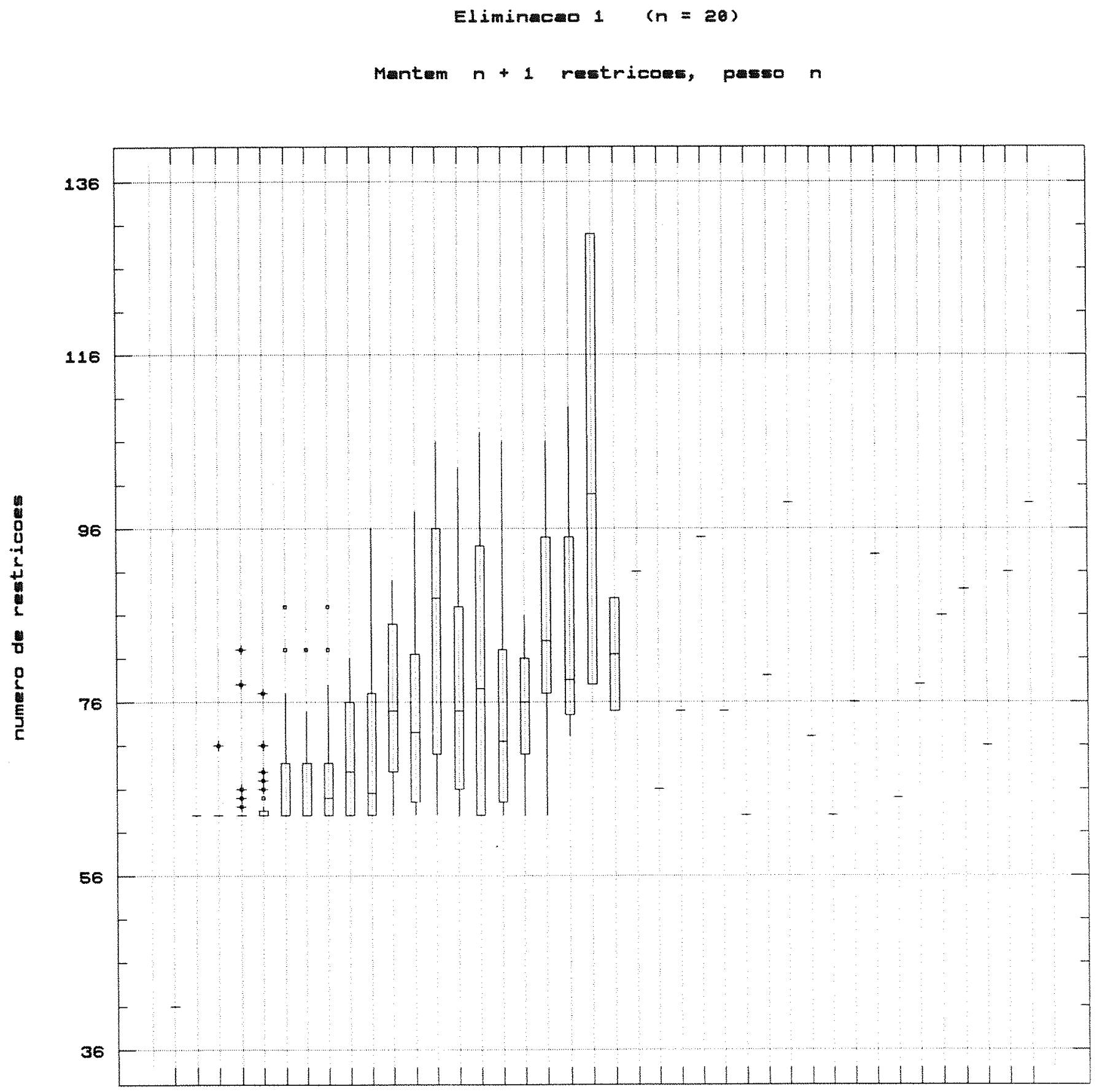

- 60100140180220260300340380420460500540580620660700740780 4080120160200240280320360400440480520560600640680720760800 
Eliminacao $1 \quad(n=20)$

Mantem $2 n$ rewtricosis, pass $n$

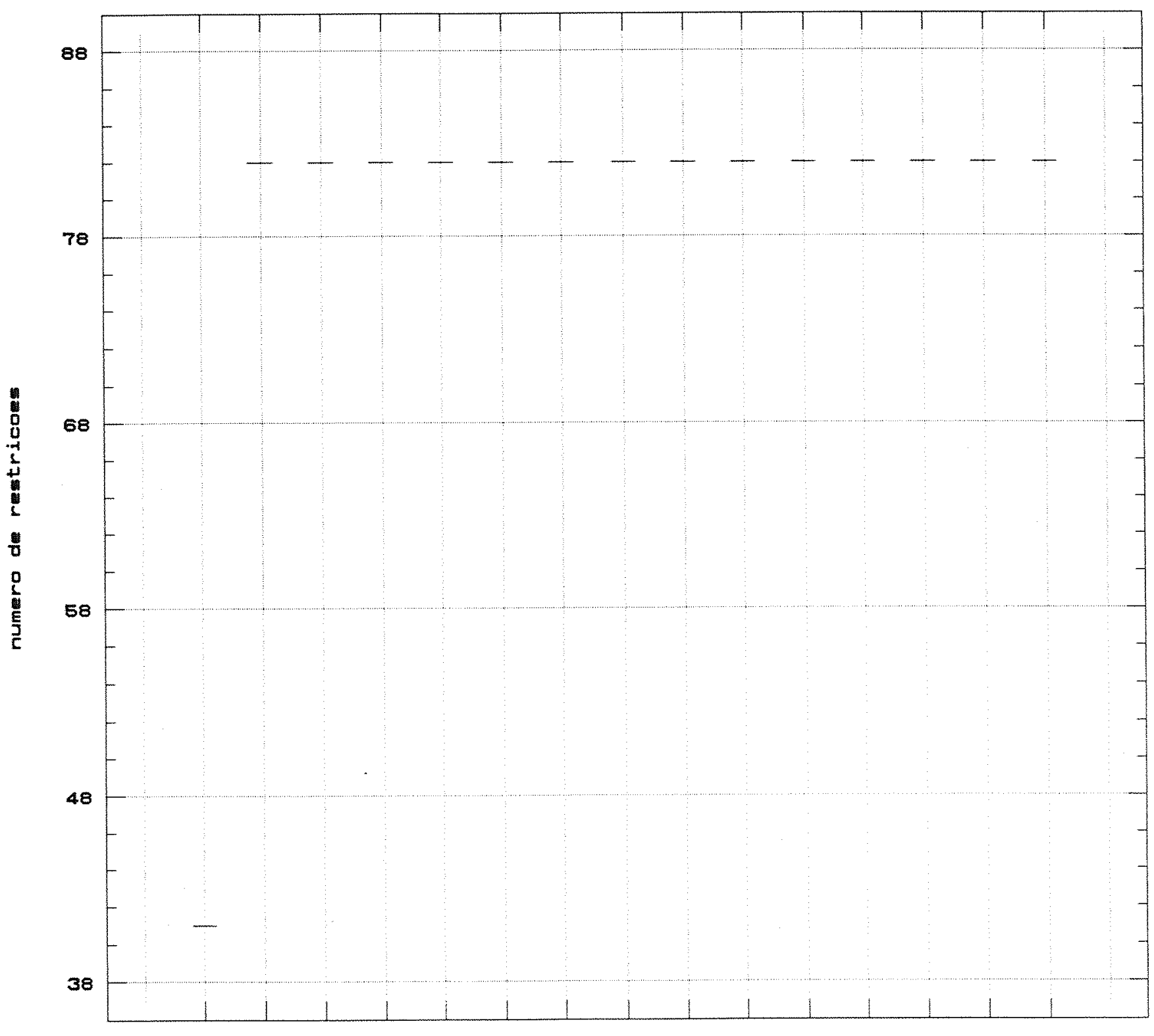

$\begin{array}{llllllllllllll}60 & 80 & 180 & 120 & 140 & 160 & 186 & 200 & 220 & 240 & 260 & 280 & 300 & 320\end{array}$

iteracoes 
Eliminacao $2 \quad(n=5)$

Distribuicao de tempo por suas variacoes

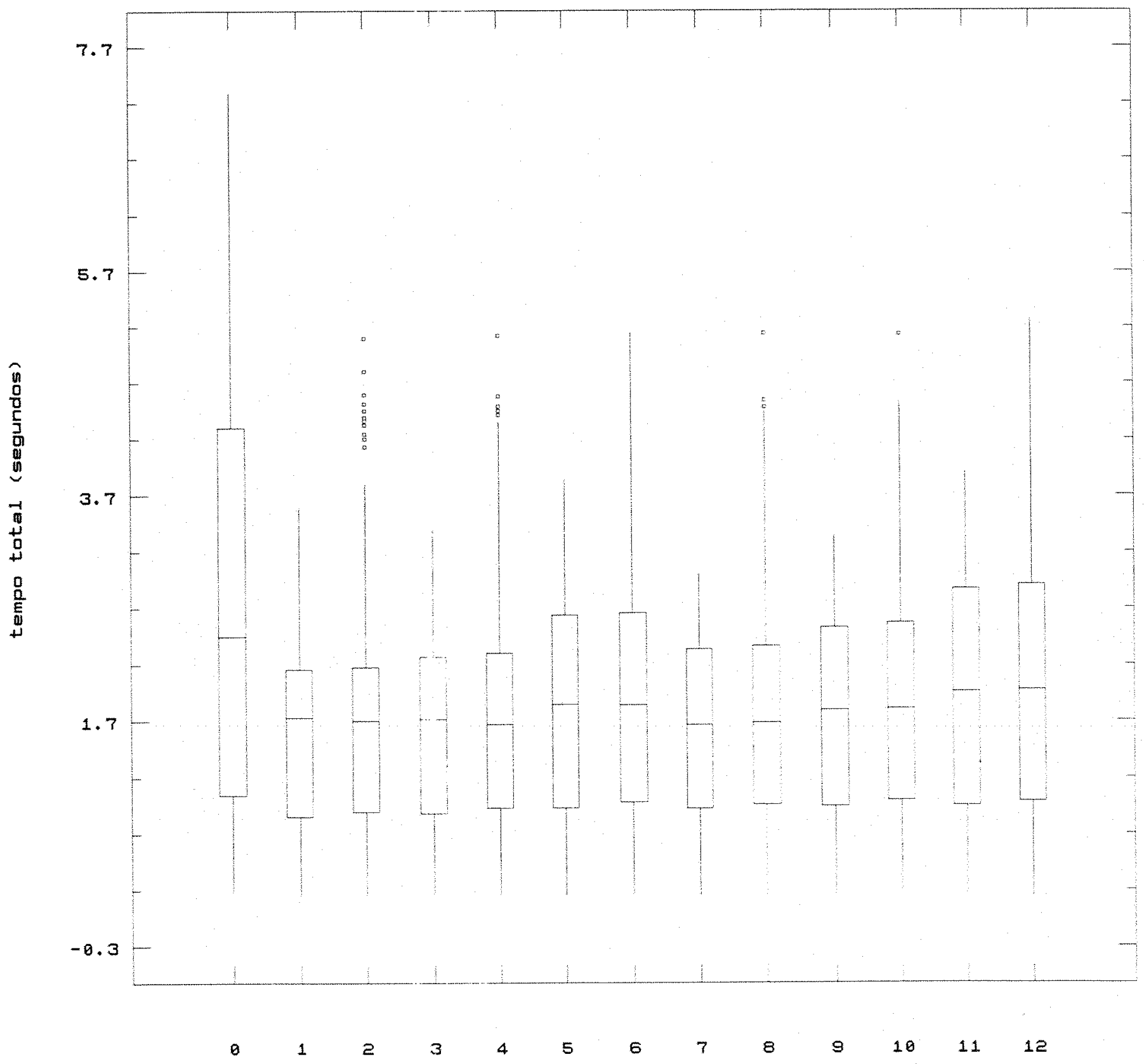

variacoes 
Eliminacao $2(n=10)$

Distribuicao de tempo por suas variacoes

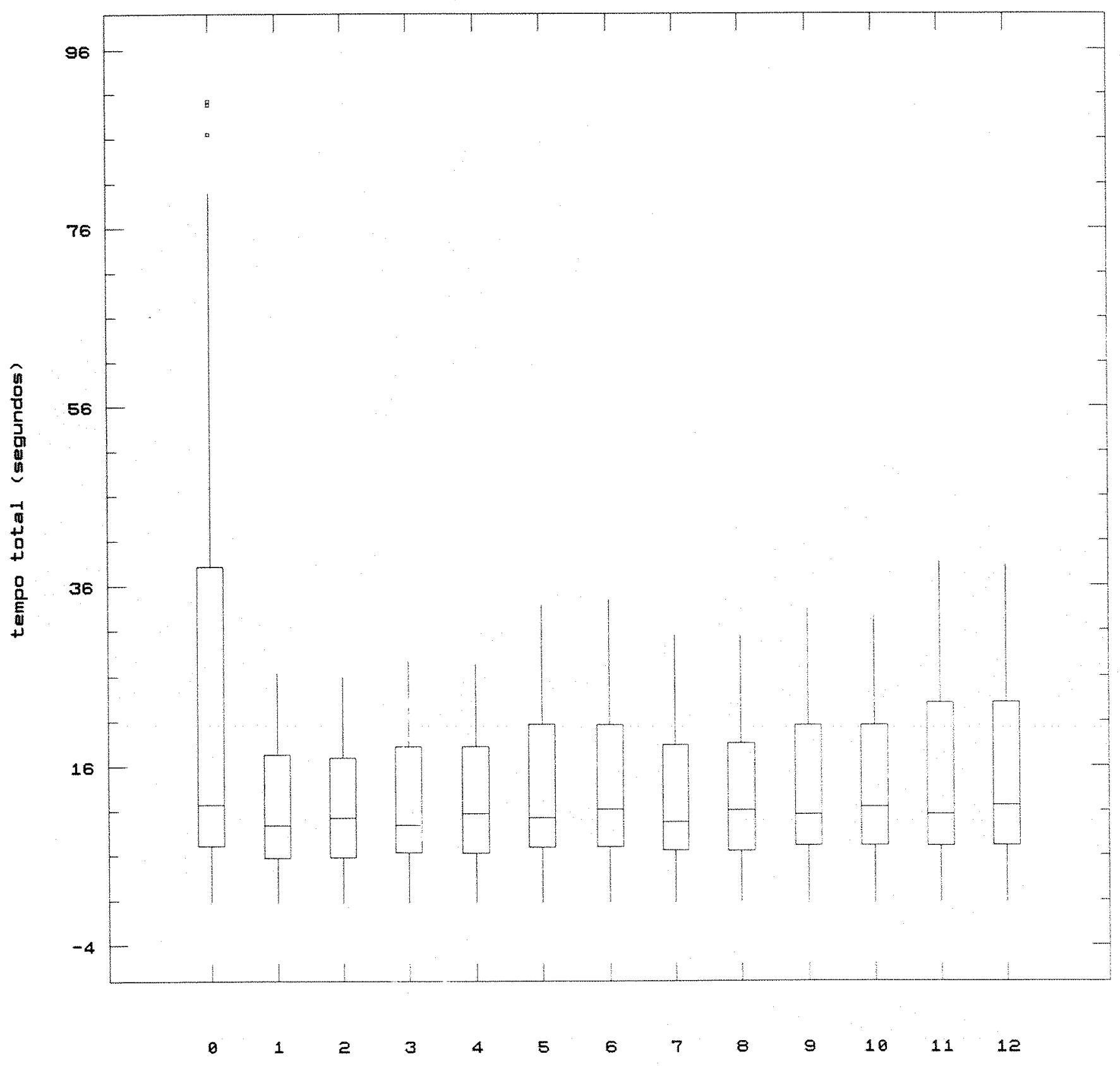

variacoes 
Eliminacao $2 \quad(n=10)$

Mantem $n+1$ restricom, pam $n$

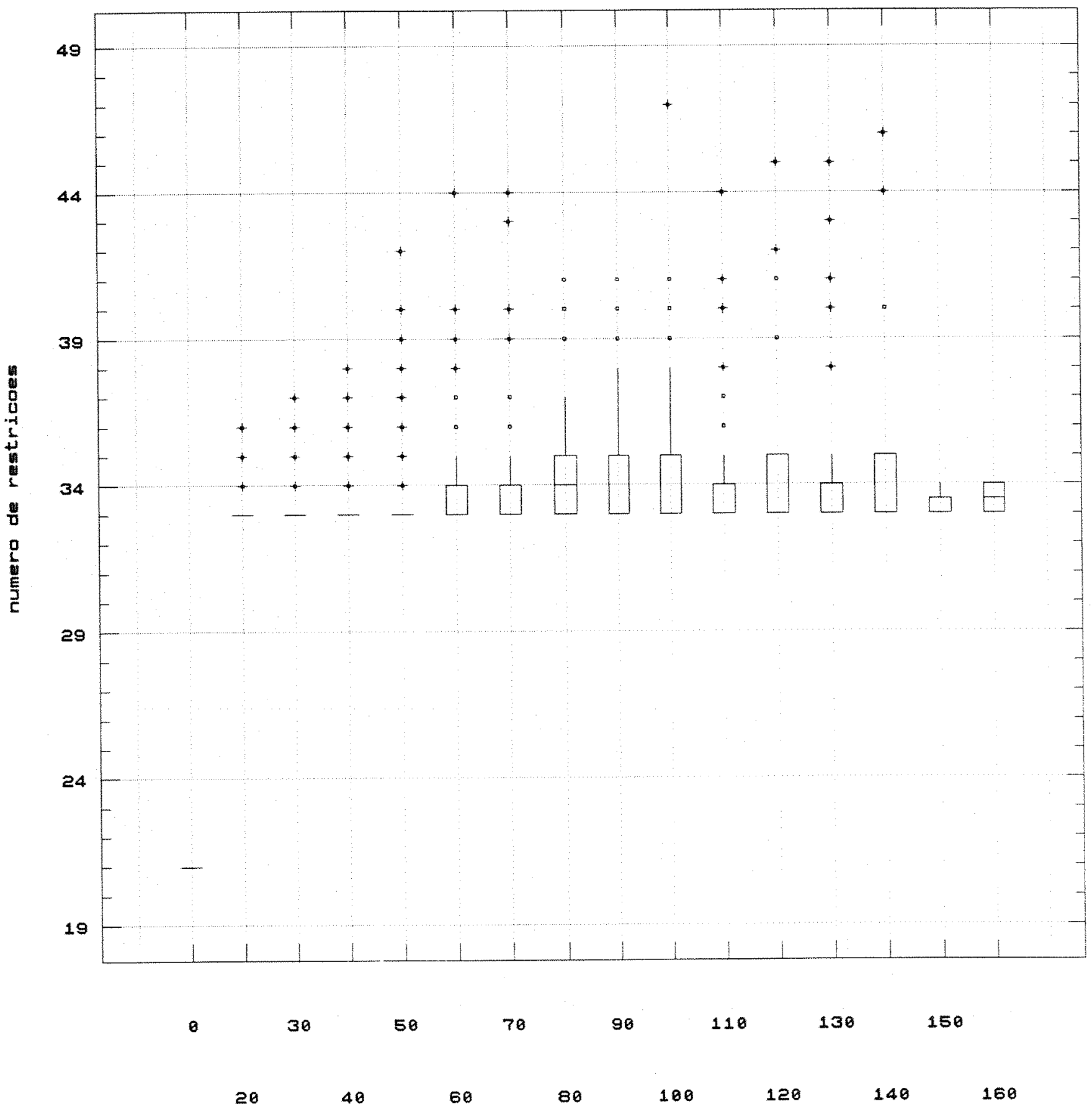




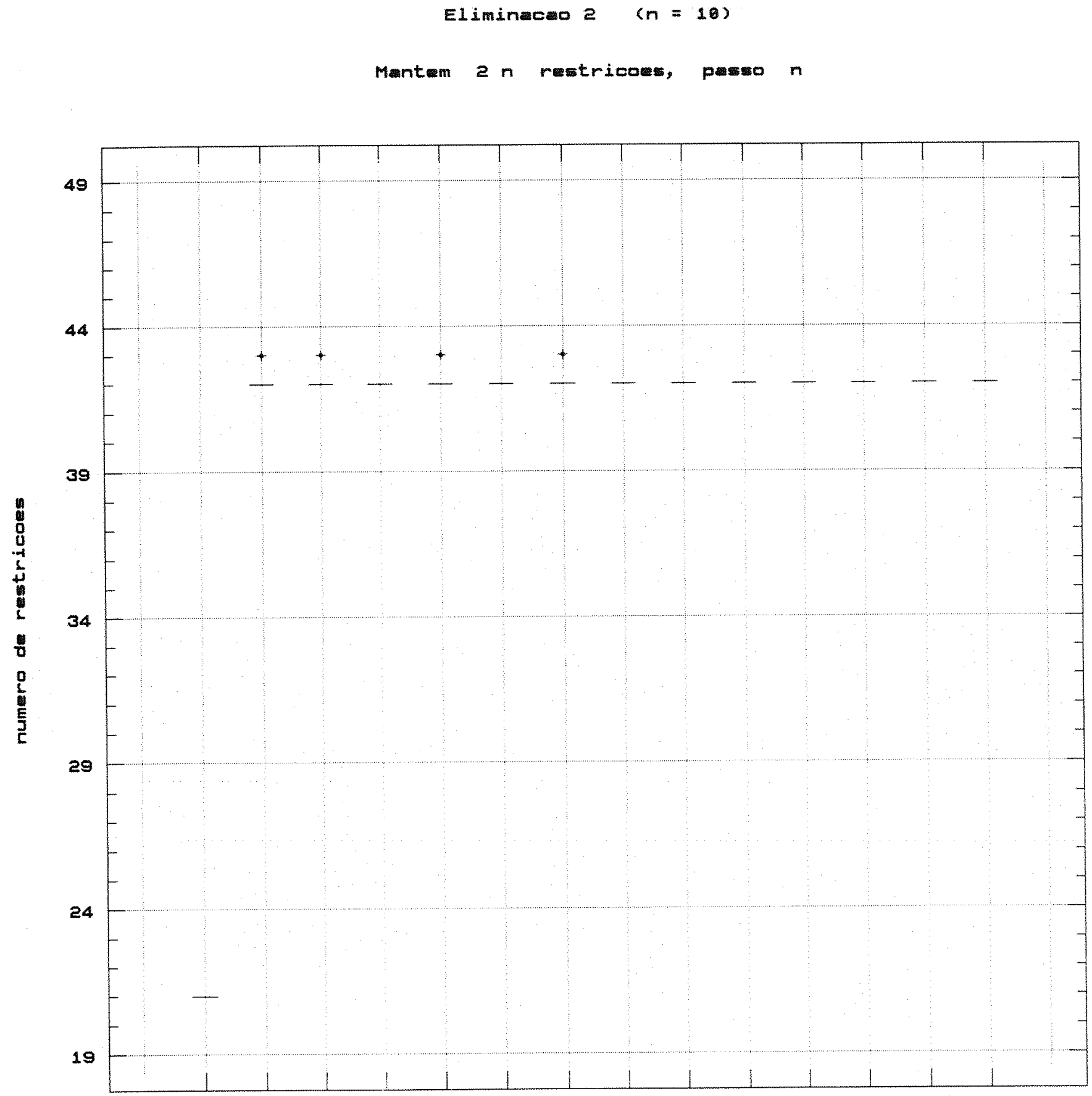

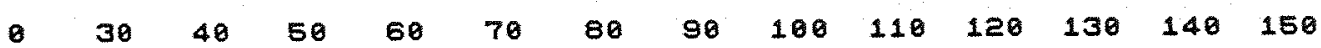


Eliminacao $2(n=5)$

Mantem 2 n restricoes, pasmo $n$

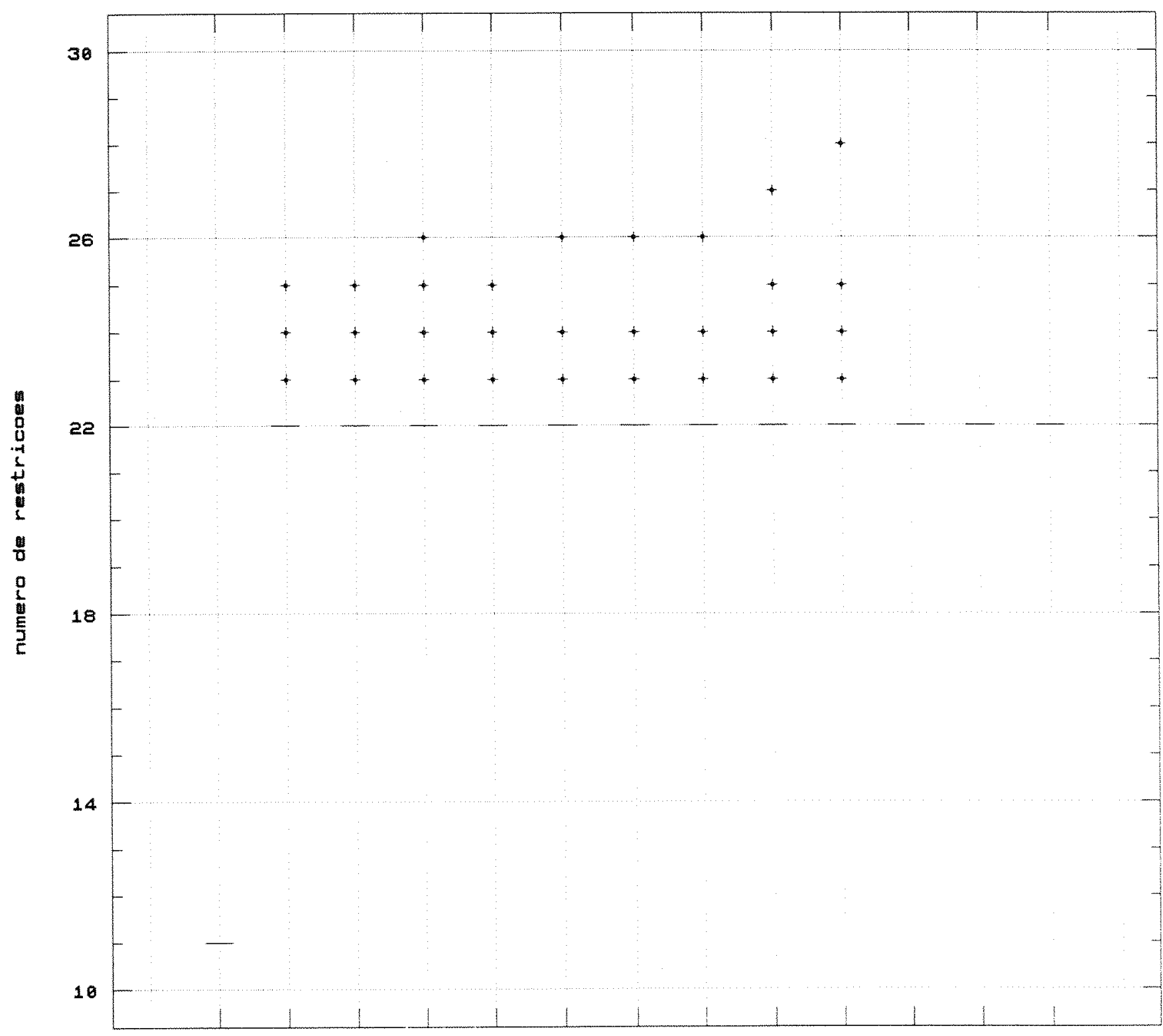

$\begin{array}{lllllllllllll}- & 15 & 20 & 25 & 30 & 35 & 40 & 45 & 50 & 55 & 60 & 65 & 70\end{array}$

iteracoes 
Eliminacia $2 \quad(n=5)$

Mantem $n+1$ restricow, pawo $n$

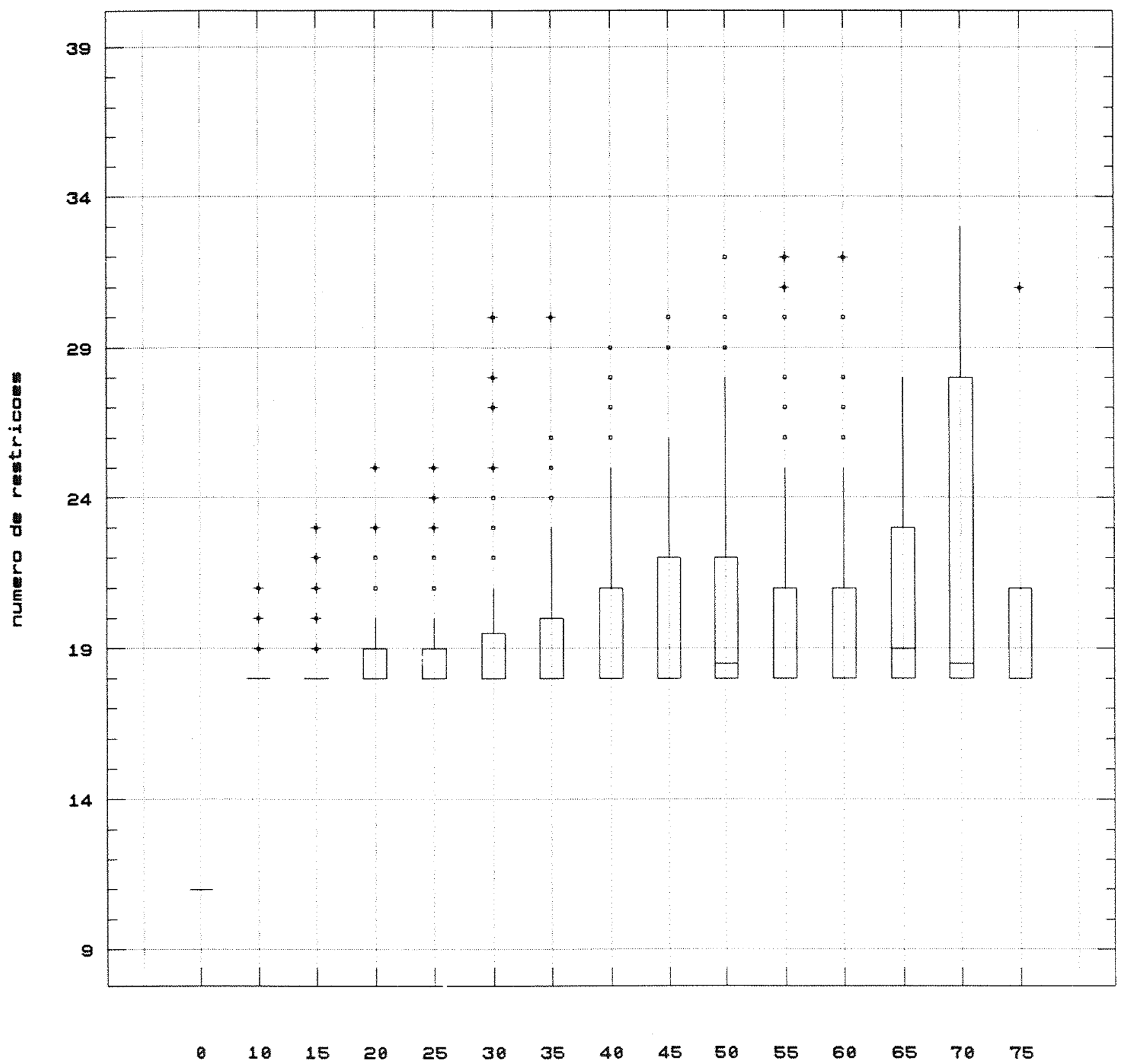




\section{Bibliografia}

[1] Dantzig, G.

Maximization of a Linear Function of Variables Subject to Linear Inequalities.

In Tj.C.Koopmans, editor, Activity Analysis of Production and Allocation, 339-347,

Wiley, New York, 1951.

[2] Delgado, A.R.S.

Utilização de Algoritmos de Pontos Interiores na Metodologia de Planos Cortantes.

Tese de Doutorado, COPPE-Universidade Federal de Rio de Janeiro, 1991.

[3] Eaves, B.C. and Zangwill, W.I.

Generalized Cutting Plane Algorithms.

SIAM J. Control, vol. 9, 529-542, 1971.

[4] Friedlander, A. e Martinez, J.M.

On the Maximization of a Concave Quadratic Function with Box Constraints.

Artigo não publicado, 1992. 
[5] Frisch, K.R.

The Logarithmic Potential Method of Convex Programming.

Memorandum, University Institute of Economics, Oslo, Norway, 1955.

[6] Goffin, J.-L. e Vial, J.-P.

Cutting planes and column generation techniques with the projective algorithm.

J. Optim. Theory Appl., 65, 409-429, 1990

[7] Gonzaga, C.C.

Comunicação pessoal, 1989

[8] Gonzaga, C.C.

Algoritmos de Pontos Interiores para Programação Linear.

17 Colóquio Brasileiro de Matemática, CNPq-IMPA, 1988.

[9] Gonzaga, C.C.

Path Following Methods For Linear Programming.

SIAM Review, vol.34,n.2.167-224, june 1992.

[10] Huard, P.

Resolution of Mathematical Programming with non linear Constraints by the method of centers.

In Non Linear Programming, J. Abadie editor, North Holland, Amsterdam, 1967.

[11] Karmarkar, N.

A New Polynomial Time Algorithm for Linear Programming.

Combinatorica, 4:373-395, 1984. 
[12] Khachiyan, L.G. A Polynomial Algorithm for Linear Programming. Soviet Mathematics Doklady, 20:191-194, 1979.

[13] Mangasarian, O.L.

Nonlinear Programming

Series in Systems Science.

McGraw-Hill, 1969.

[14] Moré, J.J. e Toraldo, G.

Algorithms for Bound Constrained Quadratic Programming Problems.

Numerische Mathematik 55, 377-400, 1989.

[15] Moré, J.J., Garbow, B.S. e Kenneth, E.H.

Testing Unconstrained Optimization Software.

ACM Transactions on Mathematical Software, Vol.7, No 1, 17-41, March 1981.

[16] Renegar, J.

A Polynomial-time Algorithm Based on Newton's Method for Linear Programming .

Mathematical Programming, vol. 40, 59-94, 1988.

[17] Rockafellar, R.T.

The Theory of Subgradients and its Applications to Problems of Optimization: Convex and Nonconvex Functions.

Research and education in Mathematics.

Berlin, Helderman Verlag, 1981.

[18] Sonnevend, G.

An Analytical Center for Polyhedrons and New Classes of Global Algorithms for Linear (Smooth, Convex) Programming . 
In Lecture Notes in Control and Information Sciences, vol. 84, 866-876, Spring Verlag, New York, NY, 1985. 